\begin{abstract}
Title of Document:

MODELING FISHERIES AGREEMENTS WITH SIDE PAYMENTS: THE CASE OF WESTERN ATLANTIC BLUEFIN TUNA

Dimitrios Reppas, Doctor of Philosophy, 2012

Directed By:

Professor Robert G. Chambers, Dept. of Agricultural and Resource Economics

Though the management of internationally shared fisheries with side payments is often considered politically difficult, quota transferring is now a policy alternative in specific fisheries treaties. Nevertheless, a theoretical framework capturing the details of the side-payments solution is, to a large extend, missing. My thesis tries to fill this gap in the literature, by proposing a static and a dynamic model in the context of a stochastic sequentially harvested stock. The conditions characterizing the solution of the dynamic model are the analogue of the Martingale Property from finance literature. The Western Atlantic Bluefin Tuna fishery is used as an example to calibrate the theory. The objective is to estimate the amount of compensation Canada should provide to the US, such that the latter restricts fishing activities. The compensation scheme could supplement/reform the existing management strategies.
\end{abstract}




\title{
MODELING FISHERIES AGREEMENTS WITH SIDE PAYMENTS: THE CASE OF WESTERN ATLANTIC BLUEFIN TUNA
}

By

\author{
Dimitrios Reppas \\ Dissertation submitted to the Faculty of the Graduate School of the \\ University of Maryland, College Park, in partial fulfillment \\ of the requirements for the degree of \\ Doctor of Philosophy \\ 2012
}

Advisory Committee:

Professor Robert G. Chambers, Chair

Professor Kenneth E. McConnell

Professor Tigran A. Melkonyan

Professor Howard D. Leathers

Professor Virginia A. Haufler 
(C) Copyright by

Dimitrios Reppas

2012 
To a friend whose efforts were denied, but words stay in my mind. 


\section{Acknowledgements}

To Bob Chambers, thank you for teaching me how to read carefully other people's research in Economics, and pushing me to arrive at the center of what is important. I shall always keep in mind what you, pretty soon, taught me, i.e., that many peopleincluding me-do not realize what they have learned although they think they do. I am afraid I still have a long way before I stop making a hundred turns about a point, wearying myself and wearying my readers. I have enjoyed every discussion with you. You are all over my Ph.D. memoirs. I will miss you saying: "Mr. Reppas: Stop making those faces and listen to me!"

To Ted McConnell, thank you for encouraging me to choose this research topic, and helping me with different stages of the calibration. It is clear to me that your pretence, sometimes, of ignorance, is nothing less than your ability to speak to every person to his own language.

To Tigran Melkonyan, thank you for supporting and inspiring me at the early steps of my research; I could not have set out without our discussions, and without you pointing out the relational contracts literature to me. Most of all, thank you for showing me how to trust young people, and how to be a caring professional. You have set these standards too high for me.

To Andreas Lange, thank you for your patience in reading previous drafts of this research, and listening to my non-structured arguments. Having you around to bother with questions, made the whole research process easier.

To Doug Lipton, thank you for your genuine enthusiasm every time I approached you for information regarding fisheries. On many occasions, you devoted personal time and effort to make my problems your problems; and provided immediate solutions, as well as relief, to me.

To Howard Leathers and Virginia Haufler, thank you for agreeing -without hesitation and on such a short notice- to become members of my committee. Thank you also for giving me feedback on my topic.

To Eirini, thank you for your continuous patience for more than 36 months now. Little did I know, when I informed you that this research needed only 6 more months to be completed, that it would take me all this time. You have been my second life throughout these last years.

To my parents, Vassilis and Eleni, as well as my sister, Olympia, thank you for always keeping faith in me. 
To Barbara Zanou, thank you for your endless phone calls supporting and encouraging me at difficult moments; for sharing your personal experiences in pursuing a Ph.D.; and for grasping my intends, even if I was thousands of miles away.

To Sarah Adelman, Chiara and Michele Baggio, Marcella Veronesi, Gustavo Guandalini, Ali Vahidnia, and Dimitrios Kapogiannis, you all have found a way to my heart.

To Matthew Larkin, Kolleen Bouchane, Mansor Aslana, Lucija Muehlenbachs, Stratis Camatsos, Katherine and Shawn Faulkner, Shinsuke Uchida, Lydia and Mike Taylor, Anmol Ratan, Vassillios Markos, Kevin Taylor, Iosif Kafkalas, Yoanna Kraus, Tremayne Jackson, and Todd Stutzman, thank you all for your wonderful support and friendship throughout the stressful years of the Ph.D. program.

I am also grateful for receiving financial support from the Department of Agricultural \& Resource Economics of the University of Maryland, the William J. Fulbright Foundation, the Harry Triantafyllou Foundation, the Louie Gerontellis Foundation, the American Hellenic Educational Progressive Association, and the George \& Victoria Karelias Foundation. 


\section{Table of Contents}

$\begin{array}{ll}\text { Dedication } & \text { ii }\end{array}$

$\begin{array}{ll}\text { Acknowledgments } & \text { iii }\end{array}$

Table of Contents $\quad$ V

$\begin{array}{ll}\text { Chapter 1: Introduction } & 1\end{array}$

Chapter 1.1: Internationally Shared Fisheries: The New Entrant Problem 2

$\begin{array}{ll}\text { Chapter 1.2: Current Management Practices } & 6\end{array}$

Chapter 1.3: Previous Economic Analysis $\quad 9$

Chapter 1.4: The Side-Payments Solution $\quad 12$

Chapter 1.4.1: Side Payments in Theory 12

Chapter 1.4.2: Side Payments in Practice 13

$\begin{array}{ll}\text { Chapter 2: Theoretical Framework } & 16\end{array}$

Chapter 2.1: The Setting: Stochastic Sequential Fishery 16

Chapter 2.2: Non-Cooperative Equilibria and Overexploitation 19

$\begin{array}{ll}\text { Chapter 2.3: Cooperation with Side Payments } & 21\end{array}$

Chapter 2.3.1: Static Model $\quad 24$

Chapter 2.3.2: Dynamic Model 29

Chapter 3: Calibration to the Western Atlantic Bluefin Tuna (WABFT) 38

$\begin{array}{ll}\text { Chapter 3.1: The WABFT Fishery } & 38\end{array}$

Chapter 3.2: The Quota Transfer Policy Alternative 42

Chapter 3.3: Sources of Data for Calibration 45

Chapter 3.4: Results of Empirical Calibration $\quad 52$ 
Chapter 4: Concluding Remarks $\quad 56$

Appendix A: The Optimal Continuation Contract for Two Periods 57

Appendix B: The Infinitely Repeated Contract 59

Figure 1: The Stochastic Sequential Fishery $\quad 61$

Figure 2: Side Payments in the Stochastic Sequential Fishery 62

Figure 3: ICCAT's Convention Area for Bluefin Tuna (separated in West and East 63 Management Area by the $45^{\circ}$ West meridian)

Figure 4: Historical Catches of Western Atlantic Bluefin Tuna (ATW) in comparison to Total Allowable Catches (TAC) set by ICCAT 63

Figure 5: CPUE-Biomass Relationships for the US Bluefin Tuna fishery 64

Figure 6: CPUE-Biomass Relationships for the Canadian Bluefin Tuna fishery 64

Figure 7: Sensitivity Analysis: K5 and K1 Specifications for alternate values of $\theta \quad 65$

Figure 8: Sensitivity Analysis: K6 and K2 Specifications for alternate values of $\theta \quad 65$

Figure 9: Sensitivity Analysis: K7 and K4 Specifications for alternate values of $\theta \quad 65$

Figure 10: Sensitivity Analysis: K5 and K1 Specifications for alternate values of the US's fishing unit cost $\quad 66$

Figure 11: Sensitivity Analysis: K6 and K2 Specifications for alternate values of the US's fishing unit cost

Figure 12: Sensitivity Analysis: K7 and K4 Specifications for alternate values of the US's fishing unit cost

Figure 13: Sensitivity Analysis: K5 and K1 Specifications for alternate values of tuna's market price

Figure 14: Sensitivity Analysis: K6 and K2 Specifications for alternate values of tuna's market price

Figure 15: Sensitivity Analysis: K7 and K4 Specifications for alternate values of tuna's market price

Table 1: Main International Fisheries Organizations by type of policy arrangement (Abundance-based catch limits, or Reciprocal harvesting) 
Table 2: Summary of Parameters and Functional Relationships for the Empirical Baseline Calibrations (US: Agent (A); Canada: Principal (P))

Table 3: Results of baseline calibration with K5 and K1 Specifications

Table 4: Results of baseline calibration with K5 and K2 Specifications

Table 5: Results of baseline calibration with K5 and K3 Specifications

Table 6: Results of baseline calibration with K6 and K1 Specifications

Table 7: Results of baseline calibration with K6 and K2 Specifications

Table 8: Results of baseline calibration with K6 and K4 Specifications

Table 9: Results of baseline calibration with K7 and K2 Specifications

Table 10: Results of baseline calibration with K7 and K3 Specifications

Table 11: Results of baseline calibration with K7 and K4 Specifications

References 


\section{Introduction}

In a review article regarding the economics of internationally shared fisheries, Munro (2007) concludes that fisheries managers are confronted with two options for finding an efficient solution to the management of such stocks: either "await the future work of applied game theorists that explore fisheries issues", or..."finish the job by eliminating the Freedom of the Seas principle and establishing defacto property rights for the charter members of fisheries organizations." My thesis contributes to the economics literature of internationally shared stocks, for the second proposed solution would be a radical one for the international legal community.

Most of the economic analysis for internationally shared fisheries has focused on cooperation without side payments, and particularly in the context of common-pool resources. Instead, I develop a theoretical framework employing side payments in a stochastic sequentially harvested stock. The side-payments solution, or quota transfers, has recently been allowed in the management of the Atlantic Bluefin Tuna. This mechanism is expected to be employed by other international organizations in which fishing nations do not have fixed property rights over the stock. A calibration exercise of the proposed theory is performed in the case of the Western Atlantic Bluefin Tuna fishery. The goal of the cali-

bration is to derive compensation estimates that could supplement the existing management strategies. 


\subsection{Internationally Shared Fisheries: The New Entrant}

\section{Problem}

According to the United Nations Convention on the Law of the Sea (United Nations $(1982,1995,2006))$, Coastal States have the property right to exclusively harvest their marine resources within 200 nautical miles from the coast, into an area called Exclusive Economic Zone ${ }^{1}$; stocks found beyond the 200 nautical miles limit, into the sea zone called Adjacent High Sea, are subject to exploitation by any country, Coastal State or not. The Adjacent High Sea is considered international waters. Non Coastal States operating in international waters are called Distant Water Fishing Nations.

Fisheries economists follow marine biologists in distinguishing among 3 classes of stocks that move across sea boundaries: transboundary, highly migratory, and straddling. Transboundary are the resources that move across two (or more) Exclusive Economic Zones; highly migratory those (mainly the tuna species) traveling from one Exclusive Economic Zone to the Adjacent High Sea; and straddling all the remaining species (other than the anadromous and catadromous $^{2}$ ) found both in the Exclusive Economic Zone and the Adjacent High Sea. There is not a clear economic, or even biological, justification for distinguishing between highly migratory and straddling stocks (Munro, 2011). The three classes are non-mutually exclusive, and the terminology seems to have been revised from time to time.

\footnotetext{
${ }^{1}$ Property rights of Coastal States were not clearly defined in the early 80's, when the United Nations Convention on the Law of the Sea was signed. The vagueness of the Convention's Article 64 allowed some nations, mainly the US, to conveniently misinterpret the Coastal States' rights regarding one category of internationally shared species: the US was pushing for an interpretation according to which Coastal States would not be granted property rights to the portion of highly migratory stocks found within Coastal States' Exclusive Economic Zones. Nevertheless, this interpretation was finally not adopted.

${ }^{2}$ Anadromous are the species that spawn in fresh water, migrate to the sea for most of their life, and return to fresh water in order to spawn and die. Catadromous species follow the opposite route, and are less important commercially. Some important anadromous species in North America are the Pacific Salmon, the Atlantic Sturgeon, and the Atlantic Stripped Bass ("Rockfish", in the Chesapeake Bay area); the Atlantic Eel is a catadromous species.
} 
Problems of overexploitation of internationally shared stocks emerged in the late 80 's. The extension of fisheries jurisdiction up to 200 nautical miles turned out to be an inefficient policy. The reason is that policy makers (in their majority, at that point, marine biologists and international lawyers) had failed to consider what economists would have pointed out in the first place, i.e., behavioral adjustments (or the Le-Châtelier Principle). ${ }^{3}$ What happened was that once Coastal States extended fisheries jurisdiction, the newly excluded Distant Water Fishing Nations responded by reallocating their effort to species unattractive until that time; the pressure simply shifted from coastal fisheries to straddling ones. Changing jurisdiction was intended to reduce overexploitation of Coastal species, but simply shifted overexploitation to internationally shared stocks.

Two well-known examples of overexploitation of internationally shared stocks in North America are the Grand Bank Groundfish stock off Newfoundland, and the Pollock fishery in Alaska. Overexploitation of the Grand Bank Groundfish stock, by Canadians and Spanish trawlers, resulted in a serious diplomatic conflict in 1992. After Canada implemented the 200 nautical miles boundary for its Exclusive Economic Zone, two segments of the Grand Bank (known as the "Nose" and the "Tail" of the Bank) were left in the Adjacent High Sea; there, the stock was open to exploitation by both Canada and the EU. Regarding the Alaskan Pollock fishery, harvests in the "Doughnut hole" (an area between the US and the Russian Exclusive Economic Zones in Alaska) were limited, until 1984, to less than 4,000 tons per year. By 1988, total harvests had reached 1,6 million tons. In 1992, US, Russia and a group of Distant Water Fishing Nations agreed upon a moratorium.

\footnotetext{
${ }^{3}$ Another example in fisheries management in which the economists' insights have been proven correct, is in the full resource utilization criterion. The central management criterion employed in the past by marine biologists was maximization of sustainable yields; however, this criterion does not find support in economic theory, for it pays attention only to the production (not cost) side of the problem. Economists helped abandoning this criterion.
} 
As a response to the crisis in internationally shared stocks, the United Nations decided, in the early 90's, to manage such stocks through Regional Fisheries Management Organizations (hereafter, for brevity, Organizations). These Organizations are coalitions of cooperating countries. Overall, the management through Organizations has been unsuccessful. For instance, the United Nations estimated that, by 2006 , approximately $2 / 3$ of all internationally shared stocks, and specifically $1 / 2$ of the world's tuna resources, had been depleted (FAO (2007)). In the US fisheries specifically, the Atlantic Salmon has been listed as an endangered species since 2000; while the moratorium on the Central Bering Pollock fishery has been in place since 1992 (NOAA (2011)). McKelvey et al. (2002) argue that Organizations, overall, may create a very destructive environment for the stock.

The main reason why Organizations have been unable to provide a satisfactory solution to the management of internationally shared stocks is free riding $^{4}$. Explicit free riding arises when a group of cooperating countries is unable to deter non-compliance (i.e., cannot impose severe penalties to those breaching the agreement), and when the group cannot deter non-participation. Non-participation occurs when a signatory decides to depart from a coalition ${ }^{5}$, or when an outsider remains a non-signatory; therefore, depleting the stock in international waters. Barrett (2005) finds that, in any type of international agreements, deterring non-participation is more difficult than deterring noncompliance. ${ }^{6}$

\footnotetext{
${ }^{4}$ Other than dealing with the problem of free riding, Munro (2001) gives another issue that needs to be resolved when forming coalitions. In the simplest case in which an Organization consists of a single Coastal State and a Distant Water Fishing Nation, the two countries need to agree on a bottom-up or top-down management approach. In the former, the preferences of the Coastal State regarding the management of the stock dominate those of the Distant Water Fishing Nation; while the opposite holds in the top-down approach.

${ }^{5}$ In practice, fishing nations are allowed to exit from coalitions after giving a short notice, typically of less than 12 months, to the Organizations.

${ }^{6}$ Also, Barrett's (1994) words capture well the explicit free-riding problem appearing in any international agreement: "the more countries agree to cooperate, the higher their collective benefits, but the higher also their incentives to free ride."
} 
Moreover, free riding in fisheries can be implicit. It is implicit when a previously non-participating fishing nation decides to accede to a coalition; therefore, reaping some of the cooperative rewards. This 'new entrant problem' makes any benefits from cooperation ephemeral. If the number of participants in Organizations could be held somehow constant, then the new entrant problem would no longer exist. 


\subsection{Current Management Practices}

Organizations try to deter free-riding by threatening with trade restrictions/import bans $^{7}$. Overall, such threats have had limited success in the past, and their efficiency has been controversial. ${ }^{8}$ The reason why, in practice, import bans have been largely ineffective is that vessels (engaging in Illegal, Unreported and Unregulated fishing ${ }^{9}$ ) can evade them by simply transferring activities to other countries (the latter called flags of convenience, or open registers ${ }^{10}$ ).

In practice, Organizations lack the legal power to remove a nation's right from harvesting in international waters; and therefore, address the implicit free riding problem. Instead, they are obliged to give a "just and reasonable" allocation of the resource to any nation "with a real interest in it" (UN (1994)). Problems also arise in dealing with explicit free-riding, because vessels are best regarded as "mobile pockets of sovereignty" in international waters; no State -other than their Flag States- can exercise jurisdiction over them. Even when

\footnotetext{
${ }^{7}$ The International Commission for the Conservation of Atlantic Tunas was the first Organization to ever impose such measures: in 1996, trade restrictions were imposed to Belize, and in 1999, to Equitorial Guinea; in both cases regarding imports of bluefin tuna. Nevertheless, these restrictions were lifted in 2002 and 2004, respectively. As of 2010, the Commission maintains sanctions to Bolivia and Georgia, regarding Bigeye tuna.

${ }^{8}$ McKelvey et al. (2003) discuss, in an economic context, the positive and negative economic consequences arising from the threat of barrier impositions.

${ }^{9}$ The term Ilegal, Unreported and Unregulated fishing was established by the Commission on the Conservation of Antarctic Marine Living Resources. This Commission desrves mentioning because it was the first one, to my knowledge, to implement an "ecosystem management approach", when the focus, in other Organizations, was on regulating single species. The Convention on the Conservation of Antarctic Marine Living Resources came into force in 1982, with a view to protecting all marine life in the Southern Ocean (excluding whales and seals, which are managed according to the International Convention for the Regulation of Whaling, and the Convention for the Conservation of the Antarctic Seals).

${ }^{10} \mathrm{An}$ open register imposes no nationality, or citizenship requirements, on those flying its flag. The owners of vessels in an open registry are not required to have any assets in the flag state; therefore, prosecution (and recovery of damages) becomes very difficult. The largest open registries are maintained by Panama, Liberia, Bahamas, Malta, Cyprus and Bermuda; other popular countries with open registries are Bolivia, Belize, Panama, and Uruguay. Moreover, "landlocked" countries, such as the Slovak Republic, and Mongolia, also offer open registries (the Singapore-based administrators of Mongolia's register advertise competitive fees, no restrictions on crew nationality, and no taxes in order to issue registrations "within the hour"). As a response to the "success" of open registers, several of the traditional maritime countries (including Netherlands, Germany, Norway, and the UK) have now set up their own second registers, offering more beneficial arrangements to ship owners.
} 
a vessel violates laws inside another country's Exclusive Economic Zone, penalties against foreign nationals cannot include imprisonment (the arrested vessel and crew have to be released on a reasonable bond); and evidence needs to be gathered in order to launch a case ${ }^{11}$.

In summary, the role of Organizations is limited to encouraging the settlement of disputes through peaceful means, and making recommendations to cooperating parties.

Recommendations have historically taken different forms. The initial practice of setting non-abundance-based catch limits has proven unsuccessful, especially in fisheries with significant variations in the spawning biomass across time $^{12}$. A well known example in which cooperation (between Canada and the US) collapsed due to an unforeseen climatic shift is the Pacific Salmon (Miller et al. $(2000,2004))^{13}$.

Today, nearly all Organizations have switched to abundance-based catch limits, i.e., catch limits that take into account the scientists' best assessment of the state of the stock. Nevertheless, it still remains a question whether catch adjustments are frequent (and accurate enough) to reflect the real conditions of the stocks.

A third type of arrangement adopted in specific fisheries is reciprocal har-

\footnotetext{
${ }^{11}$ There are examples of vessels operating illegally in Australia and the Southern Pacific that destroyed evidence prior to being arrested, even to the extent of throwing logbooks, computers, papers, and navigation equipment overboard.

12 There is a vast biological literature on the effects of natural warming-cooling cycles on fisheries, called Pacific Decadal Oscillation, North Atlantic Oscillation and El-Niño Southern Oscillation (see, for instance, Beamish et al. (2004), Vilchis et al. (2005), Perry et al. (2005), Cook and Heath (2005)). Less information is available regarding the impact of anthropogenic climate change on fisheries (see, for instance, Bakun (1990), Bakun and Weeks (2004)).

${ }^{13}$ The history of the Pacific Salmon agreements goes back to 1930, when the US and Canada signed the Fraser Treaty. This treaty was revised in 1971 and 1985, but cooperation broke down in 1993 due to climate-induced changes in the migration patterns of salmon. In 1999, after 6 years of negotiations (during which the US delegation team had to reconcile her internal differences, i.e., differences in the interests of the continental US fishers and the Alaskan US fishers), the two countries decided to sign another amendment. The main lesson from this fishery to policy makers was that conflicts could have been prevented if national quotas were abundance-based.
} 
vesting. This arrangement allows one party to access another's territory, in exchange of granting reciprocal rights to harvest. In practice, reciprocal harvesting comes with two main drawbacks: first, it requires constant monitoring of every party's activities; and second, fishers oppose to the idea of allowing foreigners deplete their national stock. Some examples of reciprocal harvesting are the 1981 and 2002 Canada-US Pacific Albacore Tuna Treaties, the 2005 EU-Norway Blue Whiting Treaty, and the 2006 EU-Russia Baltic Sea Treaty.

A "weaker" -to my mind- form of reciprocal harvesting, called Fisheries Partnership Agreement, has emerged during the last decade, where this was possible. It is "weaker" in the sense that the party "opening up" its territory requests monetary compensation, not reciprocal rights to harvest ${ }^{14}$. It was possible where African fishing nations were involved (the EU typically being the other "partner"15).

Table 1 summarizes the main international fisheries Organizations by type of policy arrangement, i.e., the use of abundance-based catch limits, or reciprocal harvesting (in its traditional, or "weak" form).

\footnotetext{
${ }^{14}$ The term "weaker" is not used formally in the literature; instead, it is my substitute for what some countries call "Partnership". The latter gives the impression that these countries have come up with some new type of arrangement; in reality, what they have found is simply a less demanding "partner".

${ }^{15}$ In May 2011, the European Commissioner for Maritime Affairs announced her intentions to continue Fisheries Partnership initiatives with a number of African nations. Previous such arrangements were established with countries such as Mozambique, Morocco, Gabon, Cape Verde, and Mauritania. Surprisingly, the EU's 1997 and 2007 arrangements (regarding blue whiting) with the Faroe Islands, a territory within the Kingdom of Denmark, were not of this nature; instead, they were pure reciprocal harvesting.
} 


\subsection{Previous Economic Analysis}

Any of the previous recommendations, abundance, or non-abundance-based catch limits, reciprocal harvesting, in either form, is reached after extensive bargaining. Even countries with a long experience in fisheries policies have had problems in reaching agreements in the past ${ }^{16}$. Analytically, the economics of internationally shared fish resources take elements from Bargaining (Cooperative, but mainly Non-Cooperative) Theory, and (Non-Cooperative) Coalition Theory.

The early literature, mainly developed by Munro $(1979,1987,1990)$, was limited to Cooperative and Non-Cooperative Games with just two players, following Nash $(1950,1951,1953)$. This literature explains the three main issues upon which the negotiating parties have to agree: the allocation of harvest shares, the optimal joint harvest rates over time, and the implementation of the agreement.

Munro (1979) argues that the second of the above issues is perhaps the toughest to be resolved in fisheries. The reason is that Coastal States will typically have different technology/harvest costs, perceptions of discount rates, and consumer tastes. If the two negotiating parties were identical in technology and consumer tastes, then the issue of deciding on the optimal joint policy would no longer exist; the equilibrium joint biomass, the equilibrium joint harvest, and the time path to these values would be calculated as in the single agent fishery dynamic problem (Clark and Munro $\left.(1975)^{17}\right)$.

\footnotetext{
${ }^{16}$ For instance, the Canada-US Pacific Hake Treaty was signed in 2003, following a longstanding disagreement since the early 90's; while the International Commission for the Conservation of the Southern Bluefin Tuna was established after 9 years of negotiations (by Australia, Japan and New Zealand).

${ }^{17}$ Clark and Munro (1975) solve the single fishery dynamic problem (based on the Schaefer model) as an autonomous (infinitely elastic demand for harvested fish and constant over time cost of effort) and linear (in the control variable) optimal control problem. The steady state biomass and harvest levels are described by the so-called "Fundamental Equation of Fisheries Economics" (which is a Modified Golden Rule Equation of capital theory), i.e., the resource's rate of return equals the social discount rate. The time path to these equilibrium values (for linear optimal control problems) is the most rapid one ("bang-bang").
} 
The main conclusions from the early literature are two: first, side payments facilitate the resolution of conflicts (compared to cooperation without transfers $)^{18}$; and second, non-cooperation does not guarantee overexploitation (Munro, 2001; Bjørndal et al., 2000), but leads to outcomes significantly inferior to those achieved under cooperation (i.e., similar to a Prisoner's Dilemma Game $)^{19}$. The inefficiency of the non-cooperative solution was also shown (following a different approach) by Levhari and Mirman $(1980)^{20}$.

The analysis becomes more elaborate by having more than two countries involved in the game. Kaitala and Munro (1997) discuss two solutions for addressing the new entrant (i.e., implicit free riding) problem: the transferable membership, and the waiting period solution. In the former, a new country becomes a member of the Organization only if a charter member first relinquishes its share; in the latter, new entrants have to go through a waiting period, before start getting a share of the cooperative surplus (this reduces the payoffs of the prospective new members, but does not necessarily change their decision to enter the fishery). Both solutions, however, are based on the assumption that the charter members of an Organization get defacto property rights of the resource.

In the late 90's, fisheries economists started employing some of the advances of Game Theory, and particularly the Characteristic- and Partition-function approach. Games in fisheries employing the Characteristic-function approach (Kaitala and Lindroos (1998), Li (1998) ${ }^{21}$ ) focus on coalition formation by more

\footnotetext{
${ }^{18}$ Munro (1979) explains, first analytically (i.e., provides a Modified Golden Rule Equation), and then graphically, the optimal policy without transfers. He then compares this policy to the "compromise harvest" policy with side payments. The latter results in one country paying the other to completely stop her activities; it is as if the resource was managed by a sole owner.

${ }^{19}$ In most cases, some sort of cooperation is necessary. There is only one in which the noncooperative equilibrium coincides with the cooperative one: when the efficient party excludes the opponent from the fishery by operating at its monopolistic optimal steady state escapement level. Hannesson (1995) calls this "fake cooperation".

${ }^{20}$ Fischer and Mirman (1996) extend Levhari and Mirman (1980) in the case of interacting species.

${ }^{21} \mathrm{Li}$ (1998) discusses the fair sharing solution to the new entrant problem. Contrary to Kaitala and Munro (1997), Li recognizes that new members can always enter an Organization; every time this happens, all countries renegotiate on the harvests shares from the grand
} 
than two harvesting nations; in these games, nevertheless, the number of players is fixed. Games in fisheries employing the Partition-function Games (Yi (1997), Pintassilgo (2003), Pintassilgo and Lindroos (2008) $)^{22}$, examine cooperation in a more realistic setting, i.e., with a non-fixed number of players.

The main conclusion from the Partition function literature is that the grand coalition with positive externalities (to the outsiders) can rarely be an equilibrium outcome; "the only equilibrium which is stable (in games characterized by positive externalities, and with more than 2 players) is the coalition structure formed by singletons" (Pintassilgo and Lindroos (2008)). Kwon (2006), in an extension of Levhari and Mirman's (1980) approach, also finds that coalitions of more than two fishing countries cannot be sustained over time.

The above findings by fisheries economists are rather pessimistic regarding the prospects of cooperation in straddling stocks. Nevertheless, this conclusion is not a big surprise, for it fits into a more general and ongoing debate among environmental economists and game theorists, namely whether the grand coalition, or small groups of coalitions, prevail in problems with international (positive or negative) externalities (Carraro and Siniscalco (1993), Funaki and Yamato (1999), Chander and Tulkens (1997) $)^{23}$.

coalition. The fair sharing rule means that each country's benefits from cooperation depend on the country's contribution towards the benefits of the grand coalition. The important assumption in Li's analysis is that all members of the grand coalition are able to agree on a binding solution. This assumption takes care of the question how small countries (who would be receiving, according to the fair sharing rule, a small share of the benefits of the grand coalition) could be prevented from reneging on the final agreement.

${ }^{22} \mathrm{Yi}$ (1997) was the first one to introduce externalities in the theory of coaltion formation by having a two stage game: in the first stage (called simultaneous move open membership game), each country (out of a finite number of players) decides whether to become a member of the Organization or not. In the second stage, the nonmembers act individually as singletons; while the members of the formed coalitions maximise their cooperative payoffs given the entire coalition structure (i.e., given the behavior of the rest of the players in other coaltions). In the second stage, a value is assigned to each coalition through a partition function; and the payoffs of each coaltion depend on the entire coalition structure.

Pintassilgo (2003) follows Yi's analysis, but does not make Yi's assumption of symmetric players.

${ }^{23}$ Carraro and Siniscalco (1993) show that agreements in problems of transfrontier pollution have two common characteristics: partial cooperation, and self-financed transfers. The authors conclude that partial cooperation, in either of its 4 forms (stable coalition commitment, sequential commitment, full cooperation minimum commitment, external commitment), could 


\subsection{The Side-Payments Solution}

\subsubsection{Side Payments in Theory}

Though most of the economic analysis examines cooperation without side payments, several authors have pointed out the superiority of policies with transfers. The reason why side payments are, in theory, preferred to cooperation without transfers, is that side payments focus on the allocation of the net economic benefits, instead of just harvests ${ }^{24}$. Munro (1979) was the first to conclude that side payments (his Compensation Principle) facilitate the resolution of conflicts. Implicit in Munro's analysis is the assumption that completely "buying-out" another country is politically acceptable. Clarke and Munro $(1987,1991)$ develop side payments between two countries, assuming that one of the parties is the sole harvester of the resource ${ }^{25}$. Laukkanen (2003) argues in favor of side payments in a stochastic sequential fishery ${ }^{26}$, but finally examines a trigger stock agreement without transfers ${ }^{27}$. McKelvey (1997) is the only one who discusses

lead to wider coalitions.

Funaki and Yamato (1999) show that the expectations of those contemplating to form a coalition, regarding the behavior of the outsiders (of the coalition), are critical for having the coalition formed or not: when the expectations are pessimistic (i.e., when everyone believes that the outsiders will act as singletons), then the grand coalition is formed (the tragedy of the commons is avoided); when the expectations are optimistic, then the grand coalition, most likely, does not form.

Chander and Tulkens (1997), similar to Funaki and Yamato (1999), find that full cooperation can be achieved ("the grand coalition belongs to the $\gamma$-core of the game"), when everyone believes that the outsiders will play as singletons (and maximize their utilities) in the event that the coalition breaks up.

${ }^{24}$ Economic benefits include the side payments.

${ }^{25}$ Clarke and Munro $(1987,1991)$ explore theoretically a form of weak reciprocal harvesting (under the assumption of perfect enforcement). The authors develop a Principal-Agent problem for a highly migratory stock, and specifically the part of the stock that falls within the Coastal State's Exclusive Economic Zone. Clarke and Munro (1987) develop the analysis under the assumption that the two parties have the same discount rate; their subsequent paper (1991) relaxes this assumption. The objective of the Principal (Coastal State) is maximization of tax revenues (taxes imposed on catches and fishing effort); the objective of the Agent (Distant Water Fishing Nation) is to maximize net revenues from harvesting the resource solely in the Principal's Exclusive Economic Zone.

${ }^{26} \mathrm{~A}$ "sequential" fishery as opposed to a "common pool" resource (a common pool fishery is harvested simultaneously by many countries).

${ }^{27}$ Laukkanen (2003) extends Hannesson's (1995) analysis to a stochastic fishery. She calculates a critical amount of recruitment, above which parties cooperate, and below which, they revert to competition for some time period. 
cooperative equilibria with side payments, specifically in the context of a sequential fishery (Hannesson (1995) discusses also the case of a sequential fishery, but without side payments $\left.{ }^{28}\right)$. However, McKelvey's analysis is preliminary, in the sense that he does not find a solution for the optimal compensation scheme as a function of some variable, and treats the problem only in a static framework ${ }^{29}$.

My thesis contributes to the economics of internationally shared fisheries by developing a more comprehensive theoretical framework for cooperation with side payments in a stochastic sequential fishery. The analysis extends to a dynamic setting. The stochastic sequential fishery is similar to that in Laukkanen (2003).

Estimating side payments in a stochastic sequential fishery (instead of a common-pool setting), shifts the economics to the world of Moral Hazard models: the amount of stock that one country leaves unharvested becomes recruitment to the other; nevertheless, recruitment is a noisy signal of the first country's (unobservable) harvesting behavior due to some environmental variability.

Before proceeding with the theoretical framework, the following Section discusses how ready policy makers are to adopt side payments in practice.

\subsubsection{Side Payments in Practice}

The evolution of institutions supporting cooperation in fisheries depends on several economic parameters ${ }^{30}$ (e.g., discount rate, cost of harvesting, maximum fishing effort-capacity, division of profits, enforcement costs), a number of

\footnotetext{
${ }^{28}$ Hannesson (1995) finds a condition for the countries' discount rates that sustains a set of cooperative equilibria without side payments in a deterministic sequential fishery. Hannesson (1997) discusses the case of a common-pool resource.

${ }^{29}$ McKelvey (1997) gives two conditions that hold for any cooperative solution: individual rationality (i.e., cooperative profits for each country are at least as great as those achieved unilaterally); and collective rationality (i.e., cooperation generates a positive surplus).

${ }^{30}$ Some of these parameters affect also the timing of signing the agreement. For example, Kaitala and Lindroos (2004) examine the conditions under which two parties prefer to start cooperating immediately, rather than keep negotiating (i.e., postpone cooperation), or never cooperate. Laukkanen (2005), contrary to Kaitala and Lindroos (2004), concludes that cooperation is sustained when countries control close to equal shares of the resource.
} 
environmental factors (e.g., initial stock size, biological growth functions, carrying capacity of the ecosystem), and the tastes/preferences of the negotiators regarding the political feasibility of the proposed cooperative solution.

Joint management with side payments has not received much attention yet in practice for two reasons: first, policy makers have traditionally viewed quota transferring as politically difficult ${ }^{31}$-but not impossible- to implement; and second, when policy makers are ready to step out of their conventional management practices and use side payments, they lack, to a large extend, the necessary tools to calculate compensation levels. My thesis attempts to address the second issue; as for the first, I believe this is an open question, the answer to which, as the following examples demonstrate, relies heavily on the culture of the negotiating parties.

The most celebrated example of side payments in multilateral marine resource cooperation is the North Pacific Fur Seal Convention, which was signed exactly one century ago, in 1911. The North Pacific Fur Seal Convention involved monetary (as well as non-monetary) transfers, and remained into force until 1984 (by that time, commercial interest for seals had disappeared): the Canadians and the Japanese decided to stop harvesting, in exchange of receiving $15 \%$ of the US's and Russia's annual harvests, or in exchange of getting $\$ 10,000$, if the latter two countries decided not to harvest in one year. ${ }^{32}$

An example in which side payments were justified on the grounds of economic efficiency but finally not employed, is the case of the Arcto-Norwegian cod. Munro (1990) finds that the Norwegians (high-cost fishers) would have been better off by completely renting out their fishery to the Russians (low-cost

\footnotetext{
${ }^{31}$ Difficult because fishers tend to be averse to the idea of foregoing a part of "their" quota.

${ }^{32}$ The North Pacific Fear Seal Treaty is a wonderful application of Coase's Theorem (though Coase wrote his paper nearly 50 years after this treaty was signed); and a reminder to Economists that agreements are not just science, but also an art (even if all assumptions of Coase's Theorem were satisfied, this treaty would not have been signedif it had not been for the excellent negotiating skills of the US President Taft). Barrett (2005) discusses, in detail, the history of the agreement, and the reasons for its success.
} 
harvesters); nevertheless, side payments were considered, at that point, totally unacceptable.

More recently, however, two Organizations allowed their members to use monetary transfers. As of 2004, members of the International Commission for the Conservation of Atlantic Tunas can adjust their national quotas through international trade (as long as the transaction happens in a transparent manner, and is endorsed by the Organization). Indeed, in 2006 and 2007, the US transferred 50 metric tones, each time, to Canada. In the case of the Pacific Salmon, the renewed 2009 treaty contains provisions for side payments (though the term side payments appears nowhere in the text of the treaty) from the US to Canada. Munro (2011) notes that the size of these payments may not be exceptionally large; however, the provisions in the renewed treaty, set a precedent within the Pacific Salmon cooperative management arrangements.

Overall, I speculate based on discussions with policy makers in the Atlantic Bluefin Tuna fishery, that though side payments are not yet widely used, they could supplement/reform the existing management strategies of Organizations. 


\section{Theoretical Framework}

The theory is developed in three Sections: Section 2.1 lays out the general setting for a sequential fish stock with stochastic stock recruitment; Section 2.2 examines the set of possible non-cooperative equilibria; and Section 2.3 explains the cooperative harvesting pattern with side payments. The analysis in Section 2.3 is developed both in a static and a dynamic setting. Side payments are used as a means to induce one country to restrict fishing activities.

\subsection{The Setting: Stochastic Sequential Fishery}

Consider the shared fish stock of Figure 1.The stock moves sequentially across three fishing areas: it spawns in a single location in Country A's Exclusive Economic Zone (EEZ); moves to Country P's EEZ to feed and mature; and then to the Adjacent High Sea (AHS) to continue its growth cycle. The matured population exhibits homing behavior, i.e., returns to A to give birth to a new spawning class. The new class, along with the old one, repeats a life cycle similar to the above. Time $t$ is measured in fish generations (therefore, Figure 1 depicts a single time period). After a couple of iterations, the overall stock consists of three age groups (larvae, juveniles, and adults).

Fishing takes place in all three areas (A's EEZ, P's EEZ, and AHS). In each area, recruitment, in period $t$, is generically denoted by $R_{t}$; harvest by $h_{t}$; and escapement (or abandonment) by $S_{t}$. Countries A and $\mathrm{P}$ are Coastal States (therefore, have exclusive property rights to harvest the stock in their territories); in the AHS, the stock is subject to exploitation by any Distant Water Fishing Nation (DWFN).

At the beginning of period $t$, and prior any harvesting takes place, both countries A and P observe the level of returning stock (from the AHS, in period $t-1$ ), and the newly-hatched juveniles in A's area. 
Thereafter, Nature makes a choice between two alternatives (States): either a random event takes place (State $(1-\theta)$ ) as A harvests the stock; or no such event occurs (State $\theta$ ). The random event is associated with stressful environmental conditions for the resource, resulting in a portion of A's stock dying unexpectedly ${ }^{33}$. When State $(1-\theta)$ occurs, recruitment in $\mathrm{A}$ is $R_{1-\theta, t}^{A}$; when State $\theta$ occurs, recruitment is $R_{\theta, t}^{A}$. For convenience, in the remaining part of Section 2, the subscript "." denotes either State of Nature.

When countries observe, at the beginning of period $t$, the level of returning stock/newly-hatched juveniles, none of them is able to infer whether the random event will occur or not. Nevertheless, they share the same beliefs about the probability of each State realizing: State $(1-\theta)$ occurs with probability $(1-\theta)$; State $\theta$ with probability $\theta$. The parameter $\theta$ takes a value between $(0,1)^{34}$.

Country A harvests her recruitment $R_{\cdot, t}^{A}$ down to an escapement level $S_{\cdot, t}^{A}=$ $R_{\cdot, t}^{A}-h_{\cdot, t}^{A} . \quad$ A's harvest decision $h_{\cdot, t}^{A}$ is unobservable to $\mathrm{P}$, and generates to Country A, fishing profits, in period $t, \pi_{t}^{A}\left(S_{\cdot, t}^{A}, R_{\cdot, t}^{A}\right)$.

The amount of stock that remains unharvested by A, migrates wholly to P's EEZ. P's final recruitment is a function of A's escapement, i.e., $R_{\cdot, t}^{P} \equiv g\left(S_{\cdot, t}^{A}\right)$, with $g$ a deterministic growth function known to both parties. This function captures the rate at which the stock feeds and matures in P's area. After the stock has reached maturity (or a minimum landing size), P extracts an amount $h_{\cdot, t}^{P 35}$. P's fishing profits, in period $t$, are $\pi_{t}^{P}\left(S_{\cdot, t}^{P}, R_{\cdot, t}^{P}\right)$, with $S_{\cdot, t}^{P}=R_{\cdot, t}^{P}-h_{\cdot, t}^{P}$ the escapement left behind by P.

P's escapement serves as recruitment to DWFNs, i.e., $S_{\cdot, t}^{P} \equiv R_{\cdot, t}^{D W F N s}$. Let

\footnotetext{
${ }^{33}$ The justification for the random event affecting only a portion of the stock (not the whole population), is that the resource consists of different age classes, some of which (perhaps the younger ones) are more vulnerable to environmental variability than others (adults).

${ }^{34}$ The reason why $\theta$ does not take the values 0 and 1 is that in a Moral Hazard framework, which will be introduced in Section 2.3, the Principal could use such a piece of information to exclude specific actions of the Agent. Moreover, the first order conditions in Sections 2.3.1 and 2.3.2 make sense only when $\theta \neq 0,1$.

${ }^{35}$ The reason why $\mathrm{P}$ starts harvesting only after the stock has reached maturity is that larger classes of fish typically fetch higher market prices per pound than smaller ones.
} 
$\lambda$ denote the percentage of stock that DWFNs harvest, i.e., $h_{\cdot, t}^{D W F N s}=\lambda *$ $R_{\cdot, t}^{D W F N s}$. The parameter $\lambda$ is strictly smaller than 1 ; this implies that it is not possible (or profitable) for DWFNs to remove the last remaining units of the resource. Moreover, for simplicity, $\lambda$ is constant over time (though the number and identities of DWFNs are expected to change). Overall, the analysis treats DWFNs as a single third party, acting opportunistically and independently of the two Coastal States ${ }^{36}$.

Finally, the escapement $S_{\cdot, t}^{D W F N s}$, left behind by DWFNs, starts its journey back to Country A. This amount of stock gives rise to the following season's recruitment, through function $F$, i.e., $F\left(S_{\cdot, t}^{D W F N s}\right) \equiv F_{\cdot, t+1}=R_{\cdot, t+1}^{A}$. This function is known to both Coastal States and captures two things: the growth rate of the returning adults while traveling in the AHS; and the newly-hatched classes in period $t+1$. Recruitment in period $t+1$ is a random variable in a similar way as recruitment in period $t$, i.e., the stock, in the beginning of period $t+1$, is potentially subject to a new random event ${ }^{37}$. For simplicity, random events are independent across time; and Coastal States do not revise their beliefs $(\theta$ and $(1-\theta))$ for the occurrence of the two States.

A new fishing cycle begins in period $t+1$, following a pattern as in period $t$.

The following Section discusses the set of non-cooperative equilibria that may emerge between the two Coastal States in this setting; Section 2.3 turns to the cooperative solution with side payments.

\footnotetext{
${ }^{36}$ I also ignore any short-term (i.e., within the same time period) adjustments in the DWFNs' fishing effort. This means that only the most efficient vessels operate in international waters; it is these vessels that (collectively) deplete the stock by a percentage $\lambda$.

${ }^{37}$ With a slight abuse of notation, $F_{., t+1}$ denotes the stock in A's area prior the new random event; while $R_{\cdot, t+1}^{A}$ denotes recruitment after Nature has selected a State in period $t+1$.
} 


\subsection{Non-Cooperative Equilibria and Overexploitation}

In a non-cooperative setting, each Coastal State maximizes its own fishing profits without taking into account the effects of her behavior on the other country's payoff function.

In the setting of Section 2.1, and assuming that the decision variable for each country is the escapement level, the steady state non-cooperative strate-

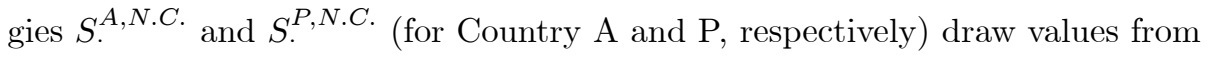
the intervals $\left[S^{A, o}, S^{A, *}\right]$ and $\left[S^{P, o}, S^{P, *}\right]$. Superscripts "o" denote the (monopolistic) suboptimal levels; superscripts " $*$ " denote the (monopolistic) optimal levels. The former are calculated by maximizing short-term profits (i.e., these are the lowest profitable abandonment levels at which countries get zero marginal profits); the latter are found by solving each country's expected discounted stream of payoffs $E\left[\sum_{t=1}^{\infty} \delta^{t} \pi_{t}^{i}\right]$, for a given/fixed strategy of the opponent ${ }^{38}(\delta$ denotes the common discount factor, $\pi_{t}^{i}=\int_{S_{., t}^{i}}^{R_{, t,}^{i}} \pi^{i}(x) d x, x=$ the in-season stock level, and $i=$ either country). ${ }^{39}$

Whether the emerging non-cooperative equilibrium allows for coexistence of fleets, or leads to exclusion of one party from the fishery (at least in the long $\left.\operatorname{run}^{40}\right)$, is an empirical issue. In the context of Section 2.1, it turns out that the difference in efficiencies of the two harvesting fleets, as well as the magnitudes of the two growth functions (i.e., $g$ and $F$ ), are critical for answering the above question.

To see this, consider first the case in which A's vessels are more efficient

\footnotetext{
${ }^{38}$ The values for the monopolistic optimal escapement levels are estimated under the assumption that there is no assymetric information regarding fishing profit functions $\pi_{t}^{i}$, i.e., under the assumption that each country knows the opponent's harvest cost function (for a fixed market price of the resource).

${ }^{39}$ McKelvey (1997) and Hannesson (1995) discuss these sets of non-cooperative equilibria, in a sequentially harvested fishery.

${ }^{40}$ I.e., after some initial adjustment period in which both countries operate.
} 
than P's (i.e., $\left.S^{A, o}<S^{P, o}\right)$. Country A excludes $\mathrm{P}$ when her strategy $S^{A, N . C \text {. }}$ satisfies the inequality $S^{A, N . C .}<g^{-1}\left(S^{P, o}\right)$. For a slow-growing resource, this inequality may by satisfied when A chooses her monopolistic optimal strategy, i.e., when $S^{A, N . C .}=S^{A, *}$. Nevertheless, for a faster growing resource (i.e., larger g) A's (preferred) strategy $S^{A, *}$ may be insufficient to exclude P; A may have to harvest more aggressively (perhaps, all the way down to her monopolistic suboptimal level $S^{A, o}$ ) to "kick" the opponent out of the fishery. A possibility also exists that $\mathrm{P}$ is never excluded, even if $\mathrm{A}$ harvests to the point where she breaks even.

A similar logic applies to the case in which P's vessels are more efficient than A's (i.e., $\left.S^{A, o}>S^{P, o}\right)$ : P's higher harvesting efficiency does not guarantee A's exclusion. Instead, exclusion is achieved when P's strategy $S^{P, N . C .}$ satisfies the inequality $S^{P, N . C .}<\frac{F^{-1}\left(S^{A, o}\right)}{1-\lambda}$, for any $S^{P, N . C .} \in\left[S^{P, o}, S^{P, *}\right]$. This inequality gives the following three comparative static results: assuming $F$ and $\lambda$ fixed, exclusion of A becomes more difficult the smaller P's comparative advantage (in harvesting the stock) is; keeping $F$ and the relative harvesting efficiencies fixed, A's exclusion becomes more difficult the less aggressive DWFNs are; and keeping $\lambda$ and relative efficiencies fixed, A's exclusion is harder the faster the resource grows in the AHS (i.e., higher $F$ ).

To sum up, the question of exclusion or not (under a non-cooperative setting) should be answered separately in each fishery that resembles that of Section 2.1. Nevertheless, equilibria that allow for coexistence of fleets are expected to be focal points. There are two reasons why exclusion seems less likely in a sequential fishery: first, (neighboring) Coastal States tend to employ similar fishing technologies (unless one is a developing country, and the other a developed one); and second, Coastal States are expected to adopt the objective of not punishing (i.e., excluding) the other, but of maximizing individual behavior/own profits ${ }^{41}$.

\footnotetext{
${ }^{41}$ Chunder and Tulkens (1997) argue, for transfrontier environmental problems, in favor of
} 


\subsection{Cooperation with Side Payments}

Consider the stochastic sequential fishery of Section 2.1, in which Countries $\mathrm{A}$ and $\mathrm{P}$ now sign an agreement/contract employing monetary transfers. The timing of the contract is the following (Figure 2 gives the corresponding flow diagram). Overall, the purpose of the agreement is to move the two Coastal States away from the non-cooperative equilibrium, towards a cooperative solution determined by $\mathrm{P}$ (however, mutually preferred).

In the beginning of period $t$, Country $\mathrm{P}$ (he) makes a take-it-or-leave-it offer to Country A (she). When P makes his offer, he is uncertain which State of Nature will occur. $\mathrm{P}$ offers monetary compensation $w_{t}$ to $\mathrm{A}$, with a view to inducing her to restrict her fishing activities. If $\mathrm{A}$ accepts the offer, $\mathrm{P}$ receives a higher level of recruitment $R_{., t}^{P}$; and therefore, harvests in excess of his noncooperative level.

After A considers P's compensation $w_{t}$ and her level of recruitment $R_{\cdot, t}^{A}$ (in either state of nature), she extracts an amount $h_{\cdot, t}^{A}\left(R_{\cdot, t}^{A}, \alpha_{t}\right)$. The variable $\alpha_{t}$ denotes A's regulatory effort to control her fishers, such that her overall harvest level is less than the non-cooperative one (the variable $\alpha_{t}$ does not refer to the Agent's fishing effort). A's regulatory effort $\alpha_{t}$, harvesting behavior $h_{\cdot, t}^{A}\left(R_{\cdot, t}^{A}, \alpha_{t}\right)$, and the realized State of Nature, remain all unobservable to $\mathrm{P}$ throughout the fishing season $t$.

A's regulatory effort $\alpha_{t}$ is a discrete variable, i.e., $0=\alpha_{t 1}<\alpha_{t 2}<\ldots<$ $\alpha_{t i}<\ldots<\alpha_{t n}=\overline{\alpha_{t}}$, and comes with an implementation cost. For simplicity, this cost is common knowledge to both parties. A is risk averse, and her overall payoff function is: $\left[u\left(\pi_{t}^{A}\left(S_{\cdot, t}^{A}, R_{\cdot, t}^{A}\right)\right)+u\left(w_{t}\right)-c\left(\alpha_{t}\right)\right]$, with $u$ denoting utility

the individual maximization behavior, rather than the vidictive behavior assumption.

Laukkanen (2003) adopts (in her fishery analysis), contrary to Chunder and Tulkens (1997), the vindictive behavior assumption (according to which both countries play at their breakeven profit levels). Nevertheless, her emerging non-cooperative equilibrium is one in which countries coexist. 
from fishing profits $\pi_{t}^{A}$, and monetary compensation $w_{t}$; and $c\left(\alpha_{t}\right)$ disutility from implementing the regulatory effort $\alpha_{t}$.

P's recruitment $R_{\cdot, t}^{P}$ (and harvest function $h_{\cdot, t}^{P}\left(R_{\cdot, t}^{P}\right)$ ) depends positively on A's level of regulatory effort $\alpha_{t}{ }^{42}$. Moreover, P's recruitment is a commonly observable variable $\left(R_{\cdot, \alpha_{t}}^{P} \equiv g\left(S_{\cdot, t}^{A}\right)\right)$; therefore, it is used as the basis for providing the compensation $w_{t}$. The overall payoff function, in period $t$, for the risk neutral Principal is: $\left[\pi_{t}^{P}\left(S_{\cdot, t}^{P}, R_{\cdot, \alpha_{t}}^{P}\right)-w_{t}\left(R_{\cdot, \alpha_{t}}^{P}\right)\right]$.

At the end of fishing season $t$, both Coastal States announce their harvest levels $h_{\cdot, t}^{A}$ and $h_{\cdot, t}^{P}$ to each other ${ }^{43}$. The contract is then executed: compensation $w_{t}$ is provided as a function of the commonly observable outcome $\left(R_{\theta, \alpha_{t}}^{P}\right.$ or $\left.R_{1-\theta, \alpha_{t}}^{P}\right)$.

The above agreement describes a Moral Hazard problem (with a discrete effort variable $\alpha_{t}$, and two possible outcomes $R_{\theta, \alpha_{t}}^{P}, R_{1-\theta, \alpha_{t}}^{P}$ for each level of effort): the Agent's action (i.e., Country A's regulatory effort $\alpha_{t}$ ) is not directly observable by the Principal (Country P); the two parties have a conflict of interests (i.e., A's regulatory effort affects her payoffs negatively ${ }^{44}$, but benefits $\mathrm{P})$; compensation $w_{t}$ is based on a commonly observable variable (i.e., $R_{\cdot, \alpha_{t}}^{P}$ ); and the observable variable is a noisy signal of A's action (i.e., $R_{\cdot, \alpha_{t}}^{P}$ aggregates A's regulatory effort and the realization of either State of Nature). This Moral Hazard setting follows the traditional formulation for hidden action problems ${ }^{45}$.

\footnotetext{
${ }^{42}$ The higher A's regulatory effort $\alpha_{t}$ is, the higher is her escapement $S_{\cdot, t}^{A}$, and thus $\mathrm{P}$ 's recruitment $R_{\cdot, t}^{P}$. For this reason, $R_{\cdot, t}^{P}$ is written, in Figure 2, with a subscript $\alpha_{t}$, i.e., as $R_{\cdot, \alpha_{t}}^{P}$.

${ }^{4}{ }^{\alpha_{t}}$ In reality, countries participating in international fisheries organizations are required to provide harvest reports within every fishing season (i.e., in-season reports). Nevertheless, these reports tend to be significanlty revised at the end of every (fishing) year; therefore, countries are unable to infer, from the interim reports, how aggresively their "opponents" behave. Only when the stock has completed a full cycle, and only when each country has harvested the resource (for that specific season), do contracting parties learn about each other's (past) harvest levels. For this reason, it makes sense to assume, in the analysis, that A's harvesting behavior $h_{\cdot, t}^{A}\left(R_{\cdot, t}^{A}, \alpha_{t}\right)$ and regulatory effort $\alpha_{t}$ are both unobservable throught the fishing season $t$.

${ }^{44}$ It affects her negatively not only through the disutility $c\left(\alpha_{t}\right)$, but also by lowering her fishing profits $\pi_{t}^{A}$.

45 "Traditional" in the sense that the States of Nature are unobservable to both parties,
} 
Section 2.3.1 solves for the conditions characterizing the optimal second best contract in a static framework; while Section 2.3.2 extends the analysis to a dynamic setting. The latter is more realistic, since the resource is a renewable one and the parties are expected to interact repeatedly over time.

Before proceeding formally with any of the analytical models (either static, or dynamic), a comment is necessary to explain why the analysis assigns the role of the Principal to Country P (and the role of the Agent to Country A). Consider momentarily the alternate scenario in which Country A (now the Principal) offers compensation to Country P (now the Agent) in order to harvest less aggressively: compensation would be a function of the Principal's output (in this case, $S_{\cdot, t}^{A}$ ), which would also be observable by the other party (since $\left.R_{\cdot, t}^{P}=g\left(S_{\cdot, t}^{A}\right)\right)$. Nevertheless, applying the above Moral Hazard analysis to this alternate scenario is no longer realistic; it is difficult to imagine now the Agent (Country P) taking a hidden action for a specific fishing season (e.g., for season $t$ ). The Agent (Country $\mathrm{P}$ ) could keep her action hidden, only if she were not announcing her harvest level at the end of the fishing season $t$. Having, in the alternate scenario, one of the two parties announcing its harvest level at the end of season $t$, while the other keeping it undisclosed, contradicts the current practice. In summary, the alternate scenario treats the two parties in a very asymmetric fashion; it makes more sense to have a model in which both countries announce catches at the end of the same fishing season ${ }^{46}$.

and the output $\left(R_{\cdot, \alpha_{t}}^{P}\right)$ is observable; contrary to the less standard approach of Chambers and Quiggin, in which the States of Nature, not the output, are observable (see, for instance, Chambers (2002)).

${ }^{46}$ The (also symmetric) case in which neither country announces catches at all, would take the analysis to a double moral hazard setting, which is beyond the purpose of my thesis. 


\subsubsection{Static Model}

This Section gives the set of first order conditions to the optimal static (spot) contract in period $t$. These conditions characterize the optimal second best regulatory effort level $\alpha_{t}^{S B}$, and the corresponding compensation schedule $w_{t}^{S B}\left(R_{,, \alpha_{t}^{S B}}^{P}\right)$ :

$$
w_{t}^{S B}\left(R_{\cdot, \alpha_{t}^{S B}}^{P}\right)=\left\{w_{t}^{S B}\left(R_{1-\theta, \alpha_{t}^{S B}}^{P}\right), w_{t}^{S B}\left(R_{\theta, \alpha_{t}^{S B}}^{P}\right)\right\}
$$

When A's regulatory effort $\alpha_{t}$ is a discrete variable $0=\alpha_{t 1}<\alpha_{t 2}<\ldots<$ $\alpha_{t i}<\ldots<\alpha_{t n}=\overline{\alpha_{t}}$, the output $R_{\cdot, \alpha_{t}}^{P}$ also takes values in a discrete set. Each level of effort $\alpha_{t}$ maps into two outputs, one for each state, and with strictly positive probabilities $(\theta \text { and }(1-\theta))^{47}$. To continue with the solution of the moral hazard problem, it is necessary to impose more structure on the set of output values, such that $\mathrm{P}$ is not certain (by simply observing an output) about the exerted level of effort $\alpha_{t}$.

The simplest possible structure for a moral hazard problem is that in which every level of observed output $R_{\cdot, t}^{P}$ (except in two cases) is derived by two adjacent levels of regulatory effort: say, by effort $\alpha_{i}$ under State $\theta$, or by effort $\alpha_{i+1}$ under State $(1-\theta)$. The two exceptions, in which the unobservability of A's effort is no longer an issue for $\mathrm{P}$, are the lowest and highest possible values of $R_{\cdot, \cdot{ }^{P}{ }^{48}}$

Assuming $\alpha_{t i}$ is the optimal level of regulatory effort, $\mathrm{P}$ solves the following

\footnotetext{
${ }^{47}$ In the typical textbook moral hazard model, every level of A's action maps into the same set of more than two outputs, with varying (for each level of effort) strictly positive probabilities. Here, however, each level of effort maps into two outputs only. This is the equivalent of a textbook model, in which every level of effort maps into the same set of more than two outputs, but with weakly positive probabilities.

${ }^{48}$ To see this, consider first A taking the lowest possible effort level $\alpha_{t 1}=0$, while State $(1-\theta)$ occurs. Then, $\mathrm{P}$ can infer A's effort level (being zero), by simply observing the outcome $R_{\cdot, \alpha_{t}}^{P} \equiv R_{1-\theta, 0}^{P}$. Similarly, when recruitment for $\mathrm{P}$ is $R_{\cdot, \alpha_{t}}^{P} \equiv R_{\theta, \bar{\alpha}_{t}}^{P}$, he knows that $\mathrm{A}$ has exerted the highest possible level of effort $\alpha_{t n}=\bar{\alpha}_{t}$, and State $\theta$ has occurred.
} 
program ${ }^{49}$, at the beginning of period $t$ (i.e., after observing the level of returning $(t-1)$ stock/newly-hatched juveniles, but before any State realizes $\left.{ }^{50}\right)$ :

$$
\max _{w_{t}\left(R_{\cdot, \alpha_{t i}}^{P}\right)}\left\{\begin{array}{c}
\theta\left\{\pi_{t}^{P}\left(S_{\theta, t}^{P}, R_{\theta, \alpha_{t i}}^{P}\right)-w_{t}\left(R_{\theta, \alpha_{t i}}^{P}\right)\right\} \\
+(1-\theta)\left\{\pi_{t}^{P}\left(S_{1-\theta, t}^{P}, R_{1-\theta, \alpha_{t i}}^{P}\right)-w_{t}\left(R_{1-\theta, \alpha_{t i}}^{P}\right)\right\}
\end{array}\right\}
$$

subject to

the Incentive Compatibility Constraints $\left(I C_{t}\right)$ :

$$
\begin{gathered}
\left\{\begin{array}{c}
\theta\left\{u\left(\pi_{t}^{A}\left(S_{\theta, t}^{A}, R_{\theta, t}^{A}\right)\right)+u\left(w\left(R_{\theta, \alpha_{t i}}^{P}\right)\right)-c\left(\alpha_{t i}\right)\right\} \\
+(1-\theta)\left\{u\left(\pi_{t}^{A}\left(S_{1-\theta, t}^{A}, R_{1-\theta, t}^{A}\right)\right)+u\left(w\left(R_{1-\theta, \alpha_{t i}}^{P}\right)\right)-c\left(\alpha_{t i}\right)\right\}
\end{array}\right\} \\
\geq\left\{\begin{array}{c}
\theta\left\{u\left(\pi_{t}^{A}\left(S_{\theta, t}^{A}, R_{\theta, t}^{A}\right)\right)+u\left(w\left(R_{\theta, \alpha_{t i^{\prime}}}^{P}\right)\right)-c\left(\alpha_{t i^{\prime}}\right)\right\} \\
+(1-\theta)\left\{u\left(\pi_{t}^{A}\left(S_{1-\theta, t}^{A}, R_{1-\theta, t}^{A}\right)\right)+u\left(w\left(R_{1-\theta, \alpha_{t i^{\prime}}}^{P}\right)\right)-c\left(\alpha_{t i^{\prime}}\right)\right\}
\end{array}\right\}
\end{gathered}
$$

for all $\alpha_{t i^{\prime}} \neq \alpha_{t i}$;

and the Individual Rationality Constraint $\left(I R_{t}\right)$ :

$$
\left\{\begin{array}{c}
\theta\left\{u\left(\pi_{t}^{A}\left(S_{\theta, t}^{A}, R_{\theta, t}^{A}\right)\right)+u\left(w\left(R_{\theta, \alpha_{t i}}^{P}\right)\right)-c\left(\alpha_{t i}\right)\right\} \\
+(1-\theta)\left\{u\left(\pi_{t}^{A}\left(S_{1-\theta, t}^{A}, R_{1-\theta, t}^{A}\right)\right)+u\left(w\left(R_{1-\theta, \alpha_{t i}}^{P}\right)\right)-c\left(\alpha_{t i}\right)\right\}
\end{array}\right\} \geq u\left(\pi_{t}^{A, N \cdot C}\left(F_{t}\right)\right)
$$

Note that this problem involves $(n-1)$ incentive compatibility constraints; and that the right hand side of the $I R_{t}$ captures A's utility from her noncooperative fishing profits. The latter are:

\footnotetext{
${ }^{49}$ The first order conditions are taken with respect to the two compensation levels, $w\left(R_{\theta, \alpha_{t i}}^{P}\right)$ and $w\left(R_{1-\theta, \alpha_{t i}}^{P}\right)$, for a given optimal effort $\alpha_{t i}$.

${ }^{50}$ The solution of the optimal contract is calculated for a specific level of $(t-1)$ returning stock/newly hatched juveniles, in A's area. In the notation of Section 2.1, the level of stock in A's area before Nature selects a State was $F_{t}$.
} 


$$
\pi_{t}^{A, N . C}\left(F_{t}\right)=\theta \pi_{t}^{A}\left(S_{\theta}^{A, N . C .}, R_{\theta, t}^{A}\right)+(1-\theta) \pi_{t}^{A}\left(S_{1-\theta}^{A, N . C \cdot}, R_{1-\theta, t}^{A}\right)
$$

$\mathrm{P}$ solves the above program for every level of regulatory effort $\alpha_{t i}$, i.e., finds the corresponding (to each effort) optimal compensation levels, $w\left(R_{\theta, \alpha_{t i}}^{P}\right)$ and $w\left(R_{1-\theta, \alpha_{t i}}^{P}\right)$, and the corresponding maximum value function $V_{t}\left(\pi_{t}^{A, N \cdot C}\left(F_{t}\right)\right)^{51}$.

The First Order conditions derived from all programs are the following.

- For the lowest possible output (realized under State $(1-\theta)$ ), i.e., for $R_{\cdot, \alpha_{t i}}^{P} \equiv R_{1-\theta, 0}^{P}$, P solves the above program with optimal level of regulatory effort $\alpha_{t i}=\alpha_{t 1}=0$. The First Order condition for the corresponding compensation is:

$$
\sum_{\kappa=2, \ldots, n} \xi_{1, \kappa}+\mu=\frac{1}{u^{\prime}\left(w\left(R_{1-\theta, 0}^{P}\right)\right)}
$$

with $\mu$ and $\xi_{1, \kappa}$ denoting, respectively, the Lagrange multipliers of the participation and the $(n-1)$ incentive compatibility constraints ${ }^{52}$;

- For the highest possible output (realized under State $\theta$ ), i.e., for $R_{\cdot, \alpha_{t i}}^{P} \equiv$ $R_{\theta, \overline{\alpha_{t}}}^{P}$, P solves the above program with optimal level of regulatory effort $\alpha_{t i}=\alpha_{t n}=\overline{\alpha_{t}}$. The First Order condition for the corresponding compensation is:

$$
\sum_{\kappa=1, \ldots, n-1} \xi_{n, \kappa}+\mu=\frac{1}{u^{\prime}\left(w\left(R_{\theta, \overline{\alpha_{t}}}^{P}\right)\right)}
$$

\footnotetext{
${ }^{51}$ The maximum value function $V_{t}$ is written for a given level of A's non-cooperative fishing profits $\pi_{t}^{A, N . C}\left(F_{t}\right)$.

${ }^{52}$ For instance, $\xi_{1,2}$ denotes the Lagrange multiplier of the incentive compatibility constraint written for the optimal effort $\alpha_{t 1}$ and the (suboptimal) effort $\alpha_{t 2} ; \xi_{1,3}$ the Lagrange multiplier of the incentive compatibility constraint written for the optimal effort $\alpha_{t 1}$ and the (suboptimal) effort $\alpha_{t 3}$, etc.
} 
with $\mu$ and $\xi_{n, \kappa}$ denoting again the Lagrange multipliers ${ }^{53}$;

- For all the remaining levels of output, i.e., for all $R_{\cdot, \alpha_{t}}^{P}$ except the two extremes $\left\{R_{1-\theta, 0}^{P}, R_{\theta, \overline{\alpha_{t}}}^{P}\right\}$, which are realized under State $\theta, \mathrm{P}$ solves the above program with optimal level of regulatory effort any $\alpha_{t i}$ except $\alpha_{t n}=$ $\overline{\alpha_{t}}$. The First Order condition for the compensation corresponding to such a $R_{\cdot, \alpha_{t}}^{P}$ is:

$$
\xi_{i, i+1}\left(2-\frac{1}{\theta}\right)+\sum_{\kappa=1, \ldots, n}^{\kappa \neq i, i+1} \xi_{i, \kappa}+\mu=\frac{1}{u^{\prime}\left(w\left(R_{\theta, \alpha_{t i}}^{P}\right)\right)}
$$

with $\mu$ and $\xi_{i, i+1}, \xi_{i, \kappa}$ denoting the Lagrange multipliers;

- For all the remaining levels of output, i.e., for all $R_{\cdot, \alpha_{t}}^{P}$ except the two extremes $\left\{R_{1-\theta, 0}^{P}, R_{\theta, \overline{\alpha_{t}}}^{P}\right\}$, which are realized under State $(1-\theta)$, P solves the above program with optimal level of regulatory effort any $\alpha_{t i}$ except $\alpha_{t 1}=0$. The First Order condition for the compensation corresponding to such a $R_{\cdot, \alpha_{t}}^{P}$ is:

$$
\xi_{i, i-1}\left(\frac{1-2 \theta}{1-\theta}\right)+\sum_{\kappa=1, \ldots, n}^{\kappa \neq i, i-1} \xi_{i, \kappa}+\mu=\frac{1}{u^{\prime}\left(w\left(R_{1-\theta, \alpha_{t i}}^{P}\right)\right)}
$$

with $\mu$ and $\xi_{i, i-1}, \xi_{i, \kappa}$ denoting the Lagrange multipliers.

Note that the last two first order conditions give different compensation values, though they refer to the same output. For instance, when $\mathrm{P}$ observes the output $R_{\theta, \alpha_{t 2}}^{P} \equiv R_{1-\theta, \alpha_{t 3}}^{P}$, he estimates one compensation value according to the third condition, i.e., from the problem with optimal effort $\alpha_{t i}=\alpha_{t 2}$; and another, according to the fourth condition, i.e., from the problem with optimal

\footnotetext{
${ }^{53}$ For instance, $\xi_{n, 2}$ denotes the Lagrange multiplier of the incentive compatibility constraint written for the optimal effort $\alpha_{t n}=\overline{\alpha_{t}}$ and the (suboptimal) effort $\alpha_{t 2}$.
} 
effort $\alpha_{t i}=\alpha_{t 3}$. The reason why $\mathrm{P}$ estimates two compensation values is that each level of output (except the two extremes ${ }^{54}$ ) is the result of either effort $\alpha_{i}$ under State $\theta$, or effort $\alpha_{i+1}$ under State $(1-\theta)$.

$\mathrm{P}$ solves a system of the above First Order conditions depending on the level of regulatory effort $0=\alpha_{t 1}<\alpha_{t 2}<\ldots<\alpha_{t i}<\ldots<\alpha_{t n}=\overline{\alpha_{t}}$ each time considered. Each system gives solutions for the compensation provided to A. In the end, he compares his maximum value function $V_{t}\left(\pi_{t}^{A, N \cdot C}\left(F_{t}\right)\right)$ from each program, and offers only the specific solution that gives him the highest expected payoffs. This solution corresponds to the optimal level of regulatory effort, i.e., it is $w_{t}^{S B}\left(R_{\cdot, \alpha_{t}^{S B}}^{P}\right)$ :

$$
w_{t}^{S B}\left(R_{\cdot, \alpha_{t}^{S B}}^{P}\right)=\left\{w_{t}^{S B}\left(R_{1-\theta, \alpha_{t}^{S B}}^{P}\right), w_{t}^{S B}\left(R_{\theta, \alpha_{t}^{S B}}^{P}\right)\right\}
$$

\footnotetext{
${ }^{54}$ For each of the extremes, i.e., for the lowest and highest possible outputs, $\mathrm{P}$ solves only one condition (the first and the second, respectivelly).
} 


\subsubsection{Dynamic Model}

Methodologically, the literature on dynamic Moral Hazard models with independent shocks across time (see, for instance, Bolton and Dewatripont (2005), or Laffont and Martimort (2002)), distinguishes between two cases: a comprehensive long-term contract without renegotiation; and a long-term contract with renegotiation.

The dynamic analysis here focuses on the former, i.e., the case of full commitment. Both parties agree on a single contract at the initial negotiation stage, and neither Country A nor P can renege. Implicit in the dynamic (as well as in the static) analysis is the assumption that the contract is court-enforceable. In other words, $R_{\cdot, \alpha_{t}}^{P}$ is not only observable but also verifiable by an outside arbiter. The role of this external third party is to impose severe penalties to any country that makes a different representation for $R_{\cdot, \alpha_{t}}^{P}$ than its true realized value.

In reality, nevertheless, Coastal States are sovereign fishing nations. Therefore, no supra-national organization could impose severe penalties to them for having breached an agreement. For this reason, Appendices A and B provide an extension to the dynamic model by relaxing the assumption of court enforceability. Cooperation, in Appendix A, extends only to two time periods, but Country A is now allowed to renege; while Appendix B examines the infinite contracting case (i.e., the most realistic of all settings).

The full commitment long-term contract without renegotiation has $\mathrm{P}$ making a take-it-or-leave-it offer at the beginning of period $t=1$. For simplicity, repetition extends only to one period, i.e., $t=1,2$. P's offer, in the beginning of period $t=1$, specifies three sets of compensation levels: the first one, $w_{1}^{*}\left(R_{\cdot, \alpha_{1}^{*}}^{P}\right)$, refers to the amount of compensation offered in the first time period $t=1$; the

other two, $w_{2}^{*}\left(R_{\cdot, \alpha_{2}^{*}}^{P} ; R_{1-\theta, \alpha_{1}^{*}}^{P}\right)$, and $w_{2}^{*}\left(R_{\cdot, \alpha_{2}^{*}}^{P} ; R_{\theta, \alpha_{1}^{*}}^{P}\right)$, to compensation offered in 
the second period $t=2$ :

$$
\begin{aligned}
w_{1}^{*}\left(R_{\cdot, \alpha_{1}^{*}}^{P}\right) & =\left\{w_{1}^{*}\left(R_{1-\theta, \alpha_{1}^{*}}^{P}\right), w_{1}^{*}\left(R_{\theta, \alpha_{1}^{*}}^{P}\right)\right\} \\
w_{2}^{*}\left(R_{\cdot, \alpha_{2}^{*}}^{P} ; R_{1-\theta, \alpha_{1}^{*}}^{P}\right) & =\left\{w_{2}^{*}\left(R_{1-\theta, \alpha_{2}^{*}}^{P} ; R_{1-\theta, \alpha_{1}^{*}}^{P}\right), w_{2}^{*}\left(R_{\theta, \alpha_{2}^{*}}^{P} ; R_{1-\theta, \alpha_{1}^{*}}^{P}\right)\right\} \\
w_{2}^{*}\left(R_{\cdot, \alpha_{2}^{*}}^{P} ; R_{\theta, \alpha_{1}^{*}}^{P}\right) & =\left\{w_{2}^{*}\left(R_{1-\theta, \alpha_{2}^{*}}^{P} ; R_{\theta, \alpha_{1}^{*}}^{P}\right), w_{2}^{*}\left(R_{\theta, \alpha_{2}^{*}}^{P} ; R_{\theta, \alpha_{1}^{*}}^{P}\right)\right\}
\end{aligned}
$$

Note that $w_{1}^{*}\left(R_{\cdot, \alpha_{1}^{*}}^{P}\right)$ is a function of the (commonly) observable output $R_{\cdot, \alpha_{1}^{*}}^{P}$ in period $t=1$; instead, the second period compensation levels are functions of the current (i.e., $R_{,, \alpha_{2}^{*}}^{P}$ ), and the past (i.e., $R_{1-\theta, \alpha_{1}^{*}}^{P}$, or $R_{\theta, \alpha_{1}^{*}}^{P}$ ) recruitment levels. The optimal contract induces A to take regulatory effort $\alpha_{1}^{*}$ in period $t=1$, and $\alpha_{2}^{*}$ in period $t=2^{55}$. The timing of the contract is the following.

At the beginning of period $t=1, \mathrm{P}$ observes the level of stock $F_{1}$ in A's $\operatorname{area}^{56}$, and makes an offer for all-periods compensation levels. If A rejects this offer, the game ends (since this is a contract without renegotiation). If she accepts, A picks her action $\alpha_{1 i}$ in the first period; both parties observe the realization of P's recruitment $R_{;, \alpha_{1 i}}^{P}$ in period $t=1$; and P provides compensation according to the terms of the contract. In the second time period, A picks her action $\alpha_{2 i}$ (based on the first period's output realization $R_{\cdot, \alpha_{1 i}}^{P}$, and the second period compensation levels); both parties observe P's recruitment level $R_{\cdot, \alpha_{2 i}}^{P}$ in period $t=2$; and compensation is offered according to the contract. The game then ends.

$\mathrm{P}$ solves for the optimal long term contract through backwards induction: he first considers the static problems in the beginning of period $t=2$, following

\footnotetext{
${ }^{55}$ The second period optimal effort $\alpha_{2}^{*}$ is a function of the first period's output realization; therefore, it should formally be written as $\alpha_{2}^{*}\left(R_{1-\theta, \alpha_{1}^{*}}^{P}\right)$, or $\alpha_{2}^{*}\left(R_{\theta, \alpha_{1}^{*}}^{P}\right)$. Nevertheless, to keep the notation simple, I drop $R_{\cdot, \alpha_{1}^{*}}$.

${ }^{56}$ Recall, from Section 2.1, that $F_{t}$ denotes the level of stock in the beginning of period $t$, i.e., prior Nature selects a State.
} 
some first period output $R_{\cdot, \alpha_{1 i}}^{P}$, and then moves backwards to the first period problem (taking into account the continuation payoffs for the second period).

The recursive procedure used to solve the long-term contract implies that the long-term contract is sequentially optimal. Since P starts solving the optimal long-term contract for a given promise (in the second period), there is no point him offering another continuation contract (other than the one he has calculated from the solution of the optimal long-term contract); so by definition, the optimal long term contract is sequentially optimal and renegotiation-proof (Laffont and Martimort (2002), p.326).

In the beginning of period $t=2, \mathrm{P}$ realizes there are two spot contracts he could offer (given the two possible States of Nature, and the corresponding outputs from period $t=1$ ): for some fixed level of regulatory effort $\alpha_{1 i}$ in the first period, either output $R_{\theta, \alpha_{1 i}}^{P}$, or output $R_{1-\theta, \alpha_{1 i}}^{P}$ realizes.

The second period spot contract following a first period output $R_{\theta, \alpha_{1 i}}^{P}$ is identical to the Static model of Section 2.3.1, with $t=2$ and:

$$
\begin{aligned}
F_{t} & =F_{\theta, \alpha_{1 i}, 2}=F\left(S_{\theta, 1}^{D W F N s}\right)=F\left((1-\lambda)\left(R_{\theta, \alpha_{1 i}}^{P}-h_{1}^{P}\right)\right) \\
\pi_{2}^{A, N . C}\left(F_{\theta, \alpha_{1 i}, 2}\right) & =\theta \pi_{2}^{A}\left(S_{\theta}^{A, N . C \cdot}, R_{\theta, 2}^{A}\right)+(1-\theta) \pi_{2}^{A}\left(S_{1-\theta}^{A, N . C .}, R_{1-\theta, 2}^{A}\right) \\
R_{\theta, 2}^{A} & =\left[F\left((1-\lambda)\left(R_{\theta, \alpha_{1 i}}^{P}-h_{1}^{P}\right)\right)\right]_{\theta, 2} \\
R_{1-\theta, 2}^{A} & =\left[F\left((1-\lambda)\left(R_{\theta, \alpha_{1 i}}^{P}-h_{1}^{P}\right)\right)\right]_{1-\theta, 2}
\end{aligned}
$$

Similarly, the second period spot contract following a first period output $R_{1-\theta, \alpha_{1 i}}^{P}$ is identical to the Static model, with $t=2$ and: 


$$
\begin{aligned}
F_{t} & =F_{1-\theta, \alpha_{1 i}, 2}=F\left(S_{1-\theta, 1}^{D W N s}\right)=F\left((1-\lambda)\left(R_{1-\theta, \alpha_{1 i}}^{P}-h_{1}^{P}\right)\right) \\
\pi_{2}^{A, N . C}\left(F_{1-\theta, \alpha_{1 i}, 2}\right) & =\theta \pi_{2}^{A}\left(S_{\theta}^{A, N . C \cdot}, R_{\theta, 2}^{A}\right)+(1-\theta) \pi_{2}^{A}\left(S_{1-\theta}^{A, N \cdot C \cdot}, R_{1-\theta, 2}^{A}\right) \\
R_{\theta, 2}^{A} & =\left[F\left((1-\lambda)\left(R_{1-\theta, \alpha_{1 i}}^{P}-h_{1}^{P}\right)\right)\right]_{\theta, 2} \\
R_{1-\theta, 2}^{A} & =\left[F\left((1-\lambda)\left(R_{1-\theta, \alpha_{1 i}}^{P}-h_{1}^{P}\right)\right)\right]_{1-\theta, 2}
\end{aligned}
$$

In the end of the second period, P's expected payoffs are either $V_{2}\left(\pi_{2}^{A, N . C}\left(F_{\theta, \alpha_{1 i}, 2}\right)\right)$ (following output $\left.R_{\theta, \alpha_{1 i}}^{P}\right)$, or $V_{2}\left(\pi_{2}^{A, N \cdot C}\left(F_{1-\theta, \alpha_{1 i}, 2}\right)\right.$ ) (following output $\left.R_{1-\theta, \alpha_{1 i}}^{P}\right)$.

Once $\mathrm{P}$ calculates the optimal continuations of the contract in the second period, he move backwards to the first: the optimal long-term contract without renegotiation solves the following program ( $\delta$ is the common discount factor; $\mathrm{P}$ is risk neutral, and $\mathrm{A}$ is risk averse, with a separable utility in time):

$\left.\left\{\begin{array}{c}\theta\left[\begin{array}{c}\left\{\pi_{1}^{P}\left(S_{\theta, 1}^{P}, R_{\theta, \alpha_{1 i}}^{P}\right)-w_{t}\left(R_{\theta, \alpha_{1 i}}^{P}\right)\right\} \\ +\delta\left\{V_{2}\left(\pi_{2}^{A, N \cdot C}\left(F_{\theta, \alpha_{1 i}, 2}\right)\right)\right\}\end{array}\right] \\ \max _{1}\left(R_{,, \alpha_{1 i}}^{P}\right) \\ \pi_{2}^{A, N . C}\left(F_{\theta, \alpha_{1 i}, 2}\right), \\ \pi_{2}^{A, N . C}\left(F_{1-\theta, \alpha_{1 i}, 2}\right)\end{array}\right\}+(1-\theta)\left[\begin{array}{c}\left\{\pi_{1}^{P}\left(S_{1-\theta, 1}^{P}, R_{1-\theta, \alpha_{1 i}}^{P}\right)-w_{1}\left(R_{1-\theta, \alpha_{1 i}}^{P}\right)\right\} \\ +\delta\left\{V_{2}\left(\pi_{2}^{A, N . C}\left(F_{1-\theta, \alpha_{1 i}, 2}\right)\right)\right\}\end{array}\right]\right\}$

subject to

the Incentive Compatibility Constraints $\left(I C^{*}\right)$ : 


$$
\begin{aligned}
& \left\{\begin{array}{c}
\theta\left[\begin{array}{c}
\left\{u\left(\pi_{1}^{A}\left(S_{\theta, 1}^{A}, R_{\theta, 1}^{A}\right)\right)+u\left(w\left(R_{\theta, \alpha_{1 i}}^{P}\right)\right)-c\left(\alpha_{1 i}\right)\right\} \\
+\delta\left\{u\left(\pi_{2}^{A, N \cdot C}\left(F_{\theta, \alpha_{1 i}, 2}\right)\right)\right\}
\end{array}\right] \\
+(1-\theta)\left[\begin{array}{c}
\left.u\left(\pi_{t}^{A}\left(S_{1-\theta, 1}^{A}, R_{1-\theta, 1}^{A}\right)\right)+u\left(w\left(R_{1-\theta, \alpha_{1 i}}^{P}\right)\right)-c\left(\alpha_{1 i}\right)\right\} \\
+\delta\left\{u\left(\pi_{2}^{A, N \cdot C}\left(F_{1-\theta, \alpha_{1 i}, 2}\right)\right)\right\}
\end{array}\right]
\end{array}\right\} \\
& \geq\left\{\begin{array}{c}
\theta\left[\begin{array}{c}
\left\{u\left(\pi_{1}^{A}\left(S_{\theta, 1}^{A}, R_{\theta, 1}^{A}\right)\right)+u\left(w\left(R_{\theta, \alpha_{1 i^{\prime}}}^{P}\right)\right)-c\left(\alpha_{1 i^{\prime}}\right)\right\} \\
+\delta\left\{u\left(\pi_{2}^{A, N \cdot C}\left(F_{\theta, \alpha_{1 i^{\prime}}, 2}\right)\right)\right\}
\end{array}\right] \\
+(1-\theta)\left[\begin{array}{c}
\left\{u\left(\pi_{t}^{A}\left(S_{1-\theta, 1}^{A}, R_{1-\theta, 1}^{A}\right)\right)+u\left(w\left(R_{1-\theta, \alpha_{1 i^{\prime}}}^{P}\right)\right)-c\left(\alpha_{1 i^{\prime}}\right)\right\} \\
+\delta\left\{u\left(\pi_{2}^{A, N \cdot C}\left(F_{1-\theta, \alpha_{1 i^{\prime}}, 2}\right)\right)\right\}
\end{array}\right]
\end{array}\right\}
\end{aligned}
$$

for all $\alpha_{1 i^{\prime}} \neq \alpha_{1 i}$;

and the Individual Rationality Constraint $\left(I R^{*}\right)$ :

$$
\left\{\begin{array}{c}
\theta\left[\begin{array}{c}
\left\{u\left(\pi_{1}^{A}\left(S_{\theta, 1}^{A}, R_{\theta, 1}^{A}\right)\right)+u\left(w\left(R_{\theta, \alpha_{1 i}}^{P}\right)\right)-c\left(\alpha_{1 i}\right)\right\} \\
+\delta\left\{u\left(\pi_{2}^{A, N \cdot C}\left(F_{\theta, \alpha_{1 i}, 2}\right)\right)\right\}
\end{array}\right] \\
+(1-\theta)\left[\begin{array}{c}
\left.u\left(\pi_{t}^{A}\left(S_{1-\theta, 1}^{A}, R_{1-\theta, 1}^{A}\right)\right)+u\left(w\left(R_{1-\theta, \alpha_{1 i}}^{P}\right)\right)-c\left(\alpha_{1 i}\right)\right\} \\
+\delta\left\{u\left(\pi_{2}^{A, N . C}\left(F_{1-\theta, \alpha_{1 i}, 2}\right)\right)\right\}
\end{array}\right]
\end{array}\right\} \geq u\left(\pi_{1}^{A, N \cdot C}\left(F_{1}\right)\right)
$$

The non-cooperative fishing profits, in the right hand side of the $\left(I R^{*}\right)$, are:

$$
\pi_{1}^{A, N . C}\left(F_{1}\right)=\theta \pi_{t}^{A}\left(S_{\theta}^{A, N . C .}, R_{\theta, 1}^{A}\right)+(1-\theta) \pi_{t}^{A}\left(S_{1-\theta}^{A, N . C .}, R_{1-\theta, 1}^{A}\right)
$$

Note that P's above objective function consists of his first period payoffs, and his expected continuation payoffs $V_{2}$. Similarly, the constraints $\left(I C^{*}\right)$ and $\left(I R^{*}\right)$ take into account A's continuation payoffs. Maximization is with respect to the first period offers, $w_{1}\left(R_{\cdot, \alpha_{1 i}}^{P}\right)=\left\{w_{1}\left(R_{1-\theta, \alpha_{1 i}}^{P}\right), w_{1}\left(R_{\theta, \alpha_{1 i}}^{P}\right)\right\}$, and the two 
"promises", $\pi_{2}^{A, N \cdot C}\left(F_{\theta, \alpha_{1 i}, 2}\right)$ and $\pi_{2}^{A, N \cdot C}\left(F_{1-\theta, \alpha_{1 i}, 2}\right) \cdot{ }^{57}$

The solution of the above program with respect to the first period optimal compensation levels $w_{1}\left(R_{\theta, \alpha_{1 i}}^{P}\right)$ and $w_{1}\left(R_{1-\theta, \alpha_{1 i}}^{P}\right)$ (for some optimal level of regulatory effort $\alpha_{1 i}$ in period $t=1$ ), resembles the solution of the Static problem (simply replace, in the First Order conditions of Section 2.3.1, where $t=1$; the Static conditions are not rewritten here).

Maximization with respect to the "promises", $\pi_{2}^{A, N . C}\left(F_{\theta, \alpha_{1 i}, 2}\right)$ and $\pi_{2}^{A, N . C}\left(F_{1-\theta, \alpha_{1 i}, 2}\right)$, yields a second set of First Order conditions. This second set of First Order conditions derived from all programs (i.e., for every level of optimal effort $\alpha_{1 i}$ ) are the following.

- For the lowest possible output in period $t=1$ (realized under State $(1-\theta))$, i.e., for $R_{\cdot, \alpha_{1 i}}^{P} \equiv R_{1-\theta, 0}^{P}, \mathrm{P}$ solves the above dynamic program with optimal level of regulatory effort $\alpha_{t i}=\alpha_{t 1}=0$. The First Order condition for the corresponding "promise" is:

$$
\sum_{\kappa=2, \ldots, n} \xi_{1, \kappa}+\mu=-V_{2}^{\prime}\left(\pi_{2}^{A, N \cdot C}\left(F_{1-\theta, 0,2}\right)\right)
$$

with $\mu$ and $\xi_{1, \kappa}$ denoting, respectively, the Lagrange multipliers of the intertemporal participation and the $(n-1)$ incentive compatibility constraints;

- For the highest possible output in period $t=1$ (realized under State $\theta$ ), i.e., for $R_{\cdot, \alpha_{1 i}}^{P} \equiv R_{\theta, \overline{\alpha_{1}}}^{P}, \mathrm{P}$ solves the above dynamic program with optimal level of regulatory effort $\alpha_{1 i}=\alpha_{1 n}=\overline{\alpha_{1}}$. The First Order condition for the corresponding "promise" is:

\footnotetext{
${ }^{57} \mathrm{~A}$ expects to get payoffs, in the end of period $t=2$, at least as much as her noncooperative fishing profits; i.e., the contract "promises" A to reach at least this level of profits (these promises are credible because of the assumption of full commitment).
} 


$$
\sum_{\kappa=1, \ldots, n-1} \xi_{n, \kappa}+\mu=-V_{2}^{\prime}\left(\pi_{2}^{A, N \cdot C}\left(F_{\theta, \overline{\alpha_{1}}, 2}\right)\right)
$$

with $\mu$ and $\xi_{n, \kappa}$ denoting again the Lagrange multipliers ${ }^{58}$;

- For all the remaining levels of output, i.e., for all $R_{\cdot, \alpha_{1 i}}^{P}$ except the two extremes $\left\{R_{1-\theta, 0}^{P}, R_{\theta, \overline{\alpha_{1}}}^{P}\right\}$, which are realized (in $t=1$ ) under State $\theta, \mathrm{P}$ solves the above dynamic program with optimal level of regulatory effort any $\alpha_{1 i}$ except $\alpha_{1 n}=\overline{\alpha_{1}}$. The First Order condition for the corresponding "promise" is:

$$
\xi_{i, i+1}\left(2-\frac{1}{\theta}\right)+\sum_{\kappa=1, \ldots, n}^{\kappa \neq i, i+1} \xi_{i, \kappa}+\mu=-V_{2}^{\prime}\left(\pi_{2}^{A, N . C}\left(F_{\theta, \alpha_{1 i}, 2}\right)\right)
$$

with $\mu$ and $\xi_{i, i+1}, \xi_{i, \kappa}$ denoting the Lagrange multipliers of the dynamic problem;

- For all the remaining levels of output, i.e., for all $R_{\cdot, \alpha_{1 i}}^{P}$ except the two extremes $\left\{R_{1-\theta, 0}^{P}, R_{\theta, \overline{\alpha_{1}}}^{P}\right\}$, which are realized (in $\left.t=1\right)$ under State $(1-$ $\theta), \mathrm{P}$ solves the above dynamic program with optimal level of regulatory effort any $\alpha_{1 i}$ except 0 . The First Order condition for the corresponding "promise" is:

$$
\xi_{i, i-1}\left(\frac{1-2 \theta}{1-\theta}\right)+\sum_{\kappa=1, \ldots, n}^{\kappa \neq i, i-1} \xi_{i, \kappa}+\mu=-V_{2}^{\prime}\left(\pi_{2}^{A, N . C}\left(F_{1-\theta, \alpha_{1 i}, 2}\right)\right)
$$

with $\mu$ and $\xi_{i, i-1}, \xi_{i, \kappa}$ denoting the Lagrange multipliers of the dynamic problem.

\footnotetext{
${ }^{58}$ These Lagrange multipliers are different from those referring to the dynamic problem with $\alpha_{t 1}=0$ the optimal level of effort.
} 
Note that the left hand side of the last four conditions are similar to the left hand side of the four conditions of the Static problem (written for $t=1$ ). Direct identification between these two sets of conditions yields, respectively:

$$
\begin{gathered}
\frac{1}{u^{\prime}\left(w_{1}\left(R_{1-\theta, 0}^{P}\right)\right)}=-V_{2}^{\prime}\left(\pi_{2}^{A, N . C}\left(F_{1-\theta, 0,2}\right)\right) \\
\frac{1}{u^{\prime}\left(w_{1}\left(R_{\theta, \overline{\alpha_{1}}}^{P}\right)\right)}=-V_{2}^{\prime}\left(\pi_{2}^{A, N . C}\left(F_{\theta, \overline{\alpha_{1}}, 2}\right)\right) \\
\frac{1}{u^{\prime}\left(w_{1}\left(R_{\theta, \alpha_{1 i}}^{P}\right)\right)}=-V_{2}^{\prime}\left(\pi_{2}^{A, N . C}\left(F_{\theta, \alpha_{1 i}, 2}\right)\right) \\
\frac{1}{u^{\prime}\left(w_{1}\left(R_{1-\theta, \alpha_{1 i}}^{P}\right)\right)}=-V_{2}^{\prime}\left(\pi_{2}^{A, N . C}\left(F_{1-\theta, \alpha_{1 i}, 2}\right)\right)
\end{gathered}
$$

The above four equations provide the main theoretical result of the thesis: a relationship between the optimal compensation $w_{1}$ in the first time period, and the optimal compensation in the second period (through the continuation payoffs $V_{2}$ ). This relationship is the equivalent of the "intertemporal consumption smoothing" (or "Martingale property") of the repeated Moral Hazard problem between an employer and an employee (Rogerson (1985)). The Martingale property is written here in the context of fisheries, for a given first-period output realization $\left(R_{1-\theta, 0}^{P}\right.$, or $R_{\theta, \overline{\alpha_{1}}}^{P}$, or $R_{\theta, \alpha_{1 i}}^{P}$, or $\left.R_{1-\theta, \alpha_{1 i}}^{P}\right)$.

The typical interpretation of the Martingale property, in a twice repeated Moral Hazard problem, is that the first period payments and their expected continuations are linked ("the optimal long-term contract with full commitment exhibits memory"); specifically, payments covary positively (i.e., a high output in the first period is rewarded not only in that period, but also in $t=2$ ). Intuitively, $\mathrm{P}$ spreads the rewards intertemporally; he does not want to give 
all rewards (necessary to induce effort) in period $t=1$, but prefers to smooth the burden of the incentive compatibility constraint (to induce effort) between today and tomorrow.

In the fishery problem, further identification of the exact link between first and second period payments requires the calculation of the derivative $V_{2}^{\prime}$. This derivative, under the structure imposed on this problem ${ }^{59}$, is the difference between the second period maximum value functions $V_{2}$ referring to two "adjacent" levels of stock (for $F_{\theta, \alpha_{1 i+1}, 2} \equiv F_{1-\theta, \alpha_{1 i}, 2}$ ):

$$
\begin{aligned}
V_{2}^{\prime}\left(\pi_{2}^{A, N . C}\left(F_{\theta, \alpha_{1 i}, 2}\right)\right) & =V_{2}\left(\pi_{2}^{A, N \cdot C}\left(F_{\theta, \alpha_{1 i+1}, 2}\right)\right)-V_{2}\left(\pi_{2}^{A, N \cdot C}\left(F_{\theta, \alpha_{1 i}, 2}\right)\right) \\
V_{2}^{\prime}\left(\pi_{2}^{A, N . C}\left(F_{1-\theta, \alpha_{1 i}, 2}\right)\right) & =V_{2}\left(\pi_{2}^{A, N \cdot C}\left(F_{\theta, \alpha_{1 i}, 2}\right)\right)-V_{2}\left(\pi_{2}^{A, N \cdot C}\left(F_{1-\theta, \alpha_{1 i}, 2}\right)\right)
\end{aligned}
$$

for any $\alpha_{1 i}$ (including 0).

Further simplification of this difference (and thus, further insights on the structure between first and second period payments) is possible only after considering specific functional relationships for the fishing profit functions.

The Principal, based on the two sets of First Order conditions in the dynamic problem, estimates, for every possible level of regulatory effort $\alpha_{1 i}$, his (intertemporal) maximum value function. Finally, he compares all his expected payoffs, and offers the contract that gives him the highest value. This is the optimal dynamic contract, denoted by $w_{1}^{*}\left(R_{\cdot, \alpha_{1}^{*}}^{P}\right)=\left\{w_{1}^{*}\left(R_{1-\theta, \alpha_{1}^{*}}^{P}\right), w_{1}^{*}\left(R_{\theta, \alpha_{1}^{*}}^{P}\right)\right\}$, $\pi_{2}^{A, N \cdot C}\left(F_{\theta, \alpha_{1}^{*}, 2}\right)$ and $\pi_{2}^{A, N \cdot C}\left(F_{1-\theta, \alpha_{1}^{*}, 2}\right)$.

\footnotetext{
${ }^{59}$ I.e., when A's effort is a discrete variable $0=\alpha_{t 1}<\alpha_{t 2}<\ldots<\alpha_{t i}<\ldots<\alpha_{t n}=\overline{\alpha_{t}}$, and when every level of observed output $R_{\cdot, t}^{P}$ (except in two cases) is derived by two adjacent levels of effort, say $\alpha_{1 i}$ and $\alpha_{1 i+1}$.
} 


\section{Calibration to the Western Atlantic Bluefin}

\section{Tuna (WABFT)}

Section 3.1 briefly describes the status of the Western Atlantic Bluefin Tuna (WABFT) fishery and the current management measures. Section 3.2 discusses the quota transfer policy alternative in this fishery; Section 3.3 presents the sources of data for deriving empirical values for the components of the theory in the case of WABFT; and Section 3.4 gives the results of the calibration.

\subsection{The WABFT Fishery}

The Atlantic Bluefin Tuna (Thunnus thynnus) is a highly migratory stock found in the Western Atlantic, Eastern Atlantic, and the Mediterranean Sea. The International Commission for the Conservation of the Atlantic Tunas (ICCAT) has been managing bluefin tuna in two groups, since $1982^{60}$ : the Eastern Atlantic/Mediterranean stock, and the Western Atlantic stock. These groups are separated by the 45 degrees West Meridian (Figure 3); and the Western Atlantic population is considerably smaller than the Eastern Atlantic/Mediterranean one $^{61}$. My thesis focuses on the Western Atlantic, because this management unit fits into the sequential fishery setting of Section 2.1.

The Western Atlantic group spans from Labrador and Newfoundland south into the Gulf of Mexico, the Caribbean Sea and the coasts off Venezuela/Brazil. Spawning of this stock is believed to occur from mid April to mid June, in the Gulf of Mexico and the Straits of Florida ${ }^{62}$. During the summer months,

\footnotetext{
${ }^{60}$ The Convention for the conservation of Atlantic Tunas was signed in 1969. Currently, ICCAT consists of 48 contracting parties.

${ }^{61}$ For instance, ICCAT's annual Total Allowable Catch, for 2011 and 2012, is set at 1750 metric tons in the Western Atlantic; versus 12900 metric tons in the Eastern Atlantic/Mediterranean.

${ }^{62}$ Other spawning areas have been hypothesized, e.g., in the mid Atlantic (Lutcavage et al. (1999)). Nevertheless, identifying clearly spawning areas is a difficult task, because tunas spawn fractionally, i.e., release eggs over several days.
} 
the stock moves northern, along the American continental shelf, with higher densities found in the Gulf of Maine, and off Nova Scotia.

Overall, the population structure of the stock remains poorly understood. Bluefin tunas exhibit homing behavior, i.e., return to their spawning grounds, typically every year ${ }^{63}$. The exact routes of this return are not completely known: movements vary across class sizes and environmental conditions. Older evidence (Mather et al. (1995)) had suggested that the stock, particularly the juveniles, when traveling back to the Gulf of Mexico, remain mainly in coastal waters (along the US/Canadian continental shelf). More recent research (Lutcavage et al. (1999), Block et al. (2005), Wilson et al. (2005)) points out that the adults (i.e., the part of the stock that is older than 10 years) first move offshore, to international waters in the North-central Atlantic (and specifically, east of the Flemish $\mathrm{Cap}^{64}$ ), and then return to the Gulf of Mexico.

The WABFT fishery has three main players ${ }^{65}$, all members of ICCAT: US, Canada, and Japan (the first two are Coastal States; the latter is a Distant Water Fishing Nation). Historically, intense fishing by these countries has reduced the abundance of the stock; as a result, the U.S., Canadian, and Japanese fisheries have been subject to strict quotas. Quotas are set by ICCAT, every 2 years, after a considerable amount of debate. ICCAT's decisions become effective 6 months after formal submission to all contracting parties.

In 1998, due to the overfished status of the stock, ICCAT initiated a 20year Western Atlantic Rebuilding Plan. The annual Total Allowable Catch was initially set at 2500 metric tons; this number has been adjusted periodically, and dropped to 1750 metric tons for 2011 and 2012. Figure 4 shows how total

\footnotetext{
${ }^{63}$ Nevertheless, bluefin tunas are not obligate annual spawners; they may exhibit the "delayed maturation" type of skipping spawning (Secor (2007)).

${ }^{64}$ The Flemish Cap lies outside Canada's Exclusive Economic Zone. The stock, there, is vulnerable to vessels from Distant Water Fishing Nations.

${ }^{65}$ There are three other smaller players: UK (in respect of Bermuda), France (in respect of St. Pierre et Miquelon), and Mexico.
} 
quotas and catches have varied in the Western Atlantic from 1982 to 200. Total catches reduced significantly during 2003-2008, due to the inability of the US to fully land her quota those years.

In the US, catches reached their highest level (since 1979) in 2002. Shortly after, the US started experiencing a reduction in catches. The annual declines in catches, during the period $2004-2008^{66}$, account for up to a $65 \%$ shortfall compared to the annual quotas set by ICCAT. In 2009, the US was able to fully utilize its quota; in 2010, however, this was not possible.

Recreational and commercial bluefin tuna fisheries in the U.S. are managed on a calendar year, and are subject to federal jurisdiction from the shoreline (i.e., State waters are also included ${ }^{67}$ ). A variety of gears is used to catch bluefin tuna ${ }^{68}$; however, about $70 \%$ of the total weight of catches comes from the Rod and Reel fishery ${ }^{69}$.

In Canada, catches have been relatively stable, around 400-600 metric tons, during the period 2002-2009 ${ }^{70}$. The main gear used in Canada is Rod and Reel, and fishers operate in several geographic areas off the Atlantic coast from July

\footnotetext{
${ }^{66}$ US catches in period 2004-2008 were $1066,848,615,858$, and 922 metric tons respectively (NOAA (2011b)).

${ }^{67}$ There are three exceptions (Maine, Connecticut, Mississippi) in which State regulations apply in State waters.

${ }^{68}$ Any vessel harvesting bluefin tuna in the US must obtain either an Atlantic Tuna Permit, or an Atlantic HMS Tuna Permit. There are 5 types of Atlantic Tuna Permits (all belong to the commercial sector): General (i.e., all hand gears: Rod and Reel, Harpoon, Handline, Bandit Gear); Harpoon; Trap; Longline; and Purse Seine. The Atlantic HMS Tuna Permits are issued in 2 categories: HMS Angling, and HMS Charter/Headboat (both categories use similar gears: Rod and Reel, Handlines, and Speargun (the latter for tunas other than BFT). The difference between the Anlging and the Charter/Headboat category is that the Charter/Headboat carries passengers for hire (i.e., passengers pay a fee); the Angling category is for private fishing). Moreover, the HMS Angling category belongs only to the recreational sector; the HMS Charter/Headboat belongs to both recreational and commercial sectors.

${ }^{69}$ The Rod and Reel fishery is by far the largest and most popular in terms of number of participants and volume of catches. It occurs mainly off the New England area, and the coasts of Virginia, North and South Carolina.

Catches from purse seiners, on the other hand, have been declining. For instance, in 2008, purse seiners' landings were 0 ; and in 2009, their landings accounted only for $1 \%$ of the total US bluefin tuna catches (while their initial allocation of the US quota was $18.8 \%$ ). The lack of availability of stock in the US, and the high operating costs in 2009 were the reasons behind the purse seiners' low catches (NOAA (2010)).

${ }^{70}$ Canadian catches peaked at 733 metric tons, in 2006 ; and dropped to 530 metric tons, in 2009.
} 
to November (when tunas have migrated into Canadian waters). The spatial distribution of the Canadian fisheries has not changed significantly during the last decade; however, there are anecdotal reports of bluefin tunas occurring in areas where they had not been observed for many years (for example, in the Baie des Chaleurs, and the western Gulf of St. Lawrence). Catch rates in the Gulf of Saint Lawrence have increased rapidly since 2004 (ICCAT (2010)).

Japanese catches have fluctuated between 300-500 metric tons; nevertheless, in 2009, they dropped to 162 metric tons. Overall, the number of Japanese longline vessels declined to about 50 in 2007, of which about 20 operated in the Western Atlantic. In 2009, there were less than 10 longline vessels targeting bluefin tuna.

The US, due to the continuing overfishing status of the stock and the shortfall in her catches, requested, in 2007, a multiyear moratorium in both sides of the Atlantic. Canada, on the other hand, suggested a moratorium only in the Eastern Atlantic. Though both proposals were backed up by scientific evidence, ICCAT largely ignored these recommendations during its 2008 meeting. This outcome was perceived with great disappointment, particularly by the US.

In 2010, ICCAT also rejected a proposal (put forward by the Principality of Monaco $^{71}$ ) to list the Atlantic Bluefin tuna under Appendix I of the Convention on International Trade in Endangered Species of Wild Fauna and Flora. This proposal would have banned international trade of bluefin tuna, and helped the stock recover. Instead, ICCAT simply expressed its commitment to suspend in the future the Western Atlantic Bluefin Tuna fisheries, if a serious threat of stock collapse becomes obvious (ICCAT $(2010 \mathrm{~b}))^{72}$.

\footnotetext{
${ }^{71}$ Delegates from the US, Canada and the EU had also supported this proposal; the Japanese lobbied successfully against it.

${ }^{72} \mathrm{~A}$ similar recommendation to suspend bluefin tuna fishing in the Eastern Atlantic/Meditteranean was issued in 2009.
} 


\subsection{The Quota Transfer Policy Alternative}

Since the US started experiencing significant reductions in her catches, US fishers were concerned that ICCAT, in one of its future meetings, may decide to transfer any unfilled US quota portion to another country (Canada, Japan, or one of the smaller players). Indeed, in 2010, ICCAT's allocation scheme for national quotas was slightly revised (ICCAT (2010b)).

Up to 2010, the US was allocated $57.48 \%$ of the total Western Atlantic quota; for 2011 and 2012 however, the US gets a smaller percentage, 54.02\%. The main difference between the two plans is that the new scheme no longer assigns a fixed tonnage to the small players (Mexico, UK, France); instead, it gives them percentages of the total quota ( $5.56 \%$ to Mexico; $0.23 \%$ to the UK; and $0.23 \%$ to France). Canada and Japan retain their historic allocations ( $22.32 \%$ and $17.64 \%$, respectively). The changes in the revised plan do not translate yet to significant losses for the US fishers (for instance, in 2010, the US baseline quota was 977 metric tons; for 2011 and 2012, it is 948 metric tons $^{73}$ ); nevertheless, they send a signal to the US regarding other countries' demands to further reduce the US's share in the future (if her shortfalls are persistent).

One of the two hypothesis the scientific community has put forward in order to explain the US's shortfalls, argues that a portion of the US stock may have shifted to northern locations (McAllister et al. $\left.(2008)^{74}\right)^{75}$.

\footnotetext{
73 The baseline quota is further adjusted for any dead discards and underharvests; the final US quota for 2011 is $858 \mathrm{mt}$ (not $948 \mathrm{mt}$ ).

${ }^{74}$ McAllister et al. (2008) compare the geolocations of satellite-tagged Bluefin tunas from 1996-2005, and conclude that a large concentration from the Carolinas has moved into the Gulf of St. Lawrence. Moreover, in 2008, large Bluefin tunas were absent in US waters. In the Gulf of St. Lawrence, the decrease in the median Bluefin tuna (from $400 \mathrm{~kg}$ to $300 \mathrm{~kg}$ ) reflects the higher abundance of youger fish.

In general, bluefin tunas are found in a wide range of ecosystems, where temperature varies between $2^{\circ}$ and $29^{\circ} \mathrm{C}$. This major variability in locations could be the result of fishing pressure and environmental parameters (for instance, local availability of adequate preys, or availability of suitable spawning conditions) fluctuating from year to year (NOAA (2010)).

${ }^{75}$ The second hypothesis is that the overall size of the population in the Western Atlantic
} 
As a response to the problem of reduced catches, fishers in the US request more lenient regulations; their landings could be maximized by relaxing domestic regulations. Though the US administration was initially reluctant to adopt such a proposal (as a long-term sustainable solution), it finally tripled (from 1 to 3 ), in 2010, the daily retention limit for large medium and giant bluefin tunas. Despite this elevated limit, the US landings were low by the end of summer 2010; therefore, in 2011, the US National Marine Fisheries Service decided to revert to the default daily limit.

I argue that a policy alternative the US may want to consider, such that she does not permanently lose (in one of ICCAT's future meetings) the unfilled portion of her quota, is a transfer to Canada. A conditional quota transfer from the US to Canada would allow the latter to exploit any extra stock he may receive from the US, i.e., any stock beyond his ICCAT share; in the same time, the US would be compensated (from Canada) for giving up some of her right to harvest the stock, in the event that she was not able to fully utilize her quota. Without such a contractual agreement, Canada, who typically abides by ICCAT's rules, would have to leave the extra stock unharvested; the stock would end up in international waters. To sum up, it is in the interest of both Coastal States (US and Canada) to jointly exploit this stock, before Japan extracts it in international waters (or before ICCAT assigns an even higher quota share to one of the smaller players in the fishery). The US fishers have also expressed their objections to the prospect of having Japan harvesting this stock: "it would be better for the stock if the quota was caught by us, than by vessels from less restrictive parties" (US Federal Register (2009), p. 26112).

The use of a quota transfer, as a policy alternative to supplement existing regulations, is already permitted by ICCAT. As of 2004, country members are allowed to transfer up to $15 \%$ of their national quotas (ICCAT (2004)) (higher has declined substantially during the last years. 
percentages are allowed for smaller players $\left.{ }^{76}\right)$. Transfers are time transactions within a given fishing year; countries cannot retransfer quotas they receive; and transfers cannot be used to cover overharvests ${ }^{77}$.

Indeed, in 2006 and 2007, the US transferred 50 metric tones, each time, to Canada. Despite the fact that US regulations (US Federal Register (2009), p. 26112) require that the potential benefits of a quota transfer should be evaluated (among other things ${ }^{78}$ ) before the transfer takes place, the amount of compensation the US fishers received, in the above two instances, was zero.

Therefore, performing a calibration exercise of the theoretical framework for side payments in the case of Western Atlantic Bluefin Tuna fishery, may provide policy makers with a rough estimate for the amount of compensation Canada should provide to the US.

\footnotetext{
${ }^{76} \mathrm{UK}$ and France can transfer up to $100 \%$ (4 metric tons) of their allocations; Mexico is allowed, for 2011 and 2012, to transfer up to $91 \%$ (86.5 metric tons) of its quota (ICCAT (2010b)).

${ }^{77}$ Overharvests in a given year are subtracted from that party's quota for the next year. If overharvests occur for any two consecutive management periods, then ICCAT recommends further reductions of that party's quota and, if necessary, trade restrictive measures.

Underharvests in a given year may be carried forward to the next year. Nevertheless, an underharvest cannot exceed $10 \%$ of the party's initial quota allocation (some exceptions apply for the small players). The US fishers have requested that the US delegation renegotiates with ICCAT this percentage (from $10 \%$ to at least $25 \%$ ).

${ }^{78} \mathrm{Such}$ as the amount of quota proposed to be transferred, the projected ability of the US vessels to harvest the total US bluefin tuna quota before the end of the fishing season, and the contracting party's ICCAT compliance status.
} 


\subsection{Sources of Data for Calibration}

This section describes the sources of data used to derive empirically the parameters for the functional relationships of Section 2 in the case of WABFT fishery. For mathematical convenience, the calibration turns to the simplest possible case of the theoretical model: the Agent chooses between two possible levels of regulatory effort, i.e., $i=1,2$ (instead of $i=1 \ldots n$, in Section 2 ); and the Principal observes one out of three possible levels of output, i.e., recruitment $R_{\cdot, \alpha_{t i}}^{P}$ takes either a low, medium, or high value (instead of $(n+1)$ possible values, in Section 2).

In summary, the components of the theoretical model for calibration are:

- the initial stock biomass $F_{t}$ in the US before the realization of either State of Nature;

- the level of recruitment $R_{\theta, t}^{A}$ in the US Exclusive Economic Zone when no random event occurs;

- the level of recruitment $R_{1-\theta, t}^{A}$ in the US when the random event occurs;

- the US's harvest function $h_{\cdot, t}^{A}\left(R_{\cdot, t}^{A}, \alpha_{t i}\right)$ for different levels of biomass and regulatory effort $\alpha_{t i}$;

- the US's harvest profit function $\pi_{t}^{A}\left(S_{\cdot, t}^{A}, R_{\cdot, t}^{A}\right)$ for different levels of escapement and biomass;

- the US's utility $u$, derived by her fishing profits and by receiving monetary compensation from Canada;

- the US's disutility $c\left(\alpha_{t i}\right)$ by implementing regulatory effort $\alpha_{t i}$;

- the US's non-cooperative fishing profits $\pi^{A, N . C .}\left(F_{t}\right)$;

- the stock growth function $g$ in Canada's Exclusive Economic Zone; 
- Canada's harvest function $h^{P}\left(R_{\cdot, \alpha_{t i}}^{P}\right)$ for different levels of biomass;

- Canada's harvest profit function $\pi_{t}^{P}\left(S_{\cdot, t}^{P}, R_{\cdot, t}^{P}\right)$ for different levels of escapement and biomass;

- the probability $(1-\theta)$ that environmental variability occurs;

- the stock's exploitation rate $\lambda$ by the Distant Water Fishing Nations (Japan);

- and the stock's growth function $F$ in the Adjacent High Sea.

The following paragraphs discuss these components in detail; while Table 2 provides a summary of the parameters and functional relationships used for the baseline calibrations.

Over the recent years, spawning biomass has fluctuated between $18-27 \%$ of 1975's level (ICCAT (2008)): for instance, in 2007, it was approximately $25 \%$ of 33000 tons (1975's level); in 2006, about $18 \%$ of this amount. For the purposes of the calibration, the smaller percentage $18 \%$ is used. Therefore, biomass $F_{t}$ in the US before the realization of either State of Nature is approximately 5940 metric tons.

The level of recruitment $R_{\theta, t}^{A}$ in the US Exclusive Economic Zone when no random event occurs takes, for simplicity, the same value as $F_{t}$, i.e. $R_{\theta, t}^{A}=5940$ metric tons.

In order to estimate the US's level of biomass $R_{1-\theta, t}^{A}$ when the random event occurs, I employ the following approach: first, I calculate the yearly percentage change in the total Western Atlantic bluefin tuna landings during the period 2004-2009 (during this period, the US was not able to land fully her assigned quotas); then I take the average of the percentage reductions; and finally I assume that the same percentage reduction applies to the abundance of the 
stock. For the purposes of the calibration, $R_{1-\theta, t}^{A}$ is approximately $90 \%$ of $F_{t}$, i.e. $R_{1-\theta, t}^{A}=5346$ metric tons. ${ }^{79}$

An econometric estimation of the production (harvest) function of either country is not possible ${ }^{80}$. The amount the US harvests $h_{\cdot, t}^{A}\left(R_{\cdot, t}^{A}, \alpha_{t i}\right)$ is a function of her biomass $R_{\cdot, t}^{A}$ and effort $\alpha_{t i}$ (to control her fishers). Finding exactly how regulatory effort $\alpha_{t i}$ affects harvests is not possible; therefore, I assign values to the US's harvests (for different combinations of regulatory effort and biomass), taking into account the following: the amount the US extracts when she takes a low level of regulatory effort $\alpha_{t 1}$ and no random event occurs corresponds approximately to her quota-not landings- during the recent years; the amount the US extracts when she takes a low level of regulatory effort $\alpha_{t 1}$ and the random event occurs corresponds approximately to her lowest realized landingsnot quotas-during the recent years; and the amount the US harvests when she takes a high level of regulatory effort $\alpha_{t 2}$ (and either State of Nature realizes) is lower than the amount under a low level of regulatory effort. For the purposes of the calibration, the following values are assigned: $h_{\theta, t}^{A}\left(R_{\theta, t}^{A}, \alpha_{t 1}\right)=1200$ metric tons; $h_{1-\theta, t}^{A}\left(R_{1-\theta, t}^{A}, \alpha_{t 1}\right)=840$ metric tons; $h_{\theta, t}^{A}\left(R_{\theta, t}^{A}, \alpha_{t 2}\right)=960$ metric tons; and $h_{1-\theta, t}^{A}\left(R_{1-\theta, t}^{A}, \alpha_{t 2}\right)=606$ metric tons. These harvest levels create a Moral Hazard problem for Canada (the US's escapement when the low regulatory effort $\alpha_{t 1}$ is exerted and no random event occurs is the same as her escapement when the high level of regulatory effort $\alpha_{t 2}$ is exerted and the random event occurs).

Similarly, for Canada, I assign values to his harvests $h_{\cdot, t}^{P}\left(R_{\cdot, \alpha_{t i}}^{P}\right)$, taking into

\footnotetext{
${ }^{79}$ The total bluefin tuna landings in the Western Atlantic for the years 2004-2009 were 2306, 2125, 1756, 1811, 1638, 2000, and 1935 metric tons respectively (ICCAT (2010), Table 1 ). Averaging across the yearly percentage reductions (a reduction of $7 \%$ for the 2003-2004 period, $17 \%$ for the $2004-2005$ period, $9.5 \%$ for the period $2006-2007$, and $3.25 \%$ for the period 2008-2009) I get a value of $9.2 \%$, or approximately $10 \%$.

${ }^{80}$ For an econometric estimation of a production function, data on biomass, effort and catches are required. ICCAT's website does not provide biomass data to proceed in this fashion. Moreover, regarding effort, ICCAT data refer to the number of fishing hrs of vessels targeting all types of tunas, not just bluefin. Therefore, extrapolating the level of effort directed to bluefin specifically, would require some assumptions on the allocation of fishing time across all species.
} 
account that higher levels of recruitment $R_{\cdot, \alpha_{t i}}^{P}$ result in higher harvests. Once the US started experiencing a shortfall in her catches, Canada's highest landings were 735 metric tons (in 2006); typically, however, Canada has been harvesting around 400 metric tons (ICCAT (2010), Table 1). For the purposes of the calibration, when Canada observes a low level of recruitment, he harvests $h_{1-\theta, t}^{P}\left(R_{1-\theta, \alpha_{t 1}}^{P}\right)=400$ metric tons; for an intermediate level of recruitment, $h_{\theta, t}^{P}\left(R_{\theta, \alpha_{t 1}}^{P}\right) \equiv h_{1-\theta, t}^{P}\left(R_{1-\theta, \alpha_{t 2}}^{P}\right)=660$ metric tons; and for the highest level of recruitment, $h_{\theta, t}^{P}\left(R_{\theta, \alpha_{t 2}}^{P}\right)=710$ metric tons.

Deriving the two fishing profit functions ( $\pi_{t}^{A}$ for the US, and $\pi_{t}^{P}$ for Canada), has been the most challenging part of this exercise. The US profit function is finally estimated in the form $\pi_{t}^{A}\left(h_{\cdot, t}^{A}, R_{\cdot, t}^{A}\right)=\tau * h_{\cdot, t}^{A}-T C^{A}\left(h_{\cdot, t}^{A}, R_{\cdot, t}^{A}\right)$, with $\tau$ denoting the ex-vessel price of bluefin tuna, and $T C^{A}$ the US's harvest cost function. Similarly, Canada's fishing profit function is $\pi_{t}^{P}\left(h_{\cdot, t}^{P}, R_{\cdot, t}^{P}\right)=\tau * h_{\cdot, t}^{P}-$ $T C^{P}\left(h_{\cdot, t}^{P}, R_{\cdot, t}^{P}\right)$, with $T C^{P}$ Canada's harvest cost function.

Notice that the price $\tau$ of bluefin tuna in the above fishing profit functions is fixed, because more than half of the US catches, and about $80 \%$ of the Canadian catches, are exported to the Japanese market. Western Atlantic Bluefin tunas are immediately gutted, upon landing, and either refrigerated or exported (in crates filled with ice) within a few hours to Japan (NOAA (2008)). During the period 1996-2001, the US was exporting approximately $85 \%$ of her bluefin tuna catches to Japan (some of them were re-exports from Mexico), but this percentage decreased to $51 \%$ in 2009 (NOAA (2010)). The recent decrease in the US exports may be the result of the reduced availability of bluefin in the US, and the development of a domestic market for high quality sushi. For the purposes of the baseline calibrations, the market price of tuna $\tau$ is set at $\$ 20$ per $\mathrm{kg}$ (about $\$ 9$ per pound). In the US market, the average ex-vessel price per lb, in 2009 , was $\$ 7.09$ in the North Atlantic, $\$ 9.40$ in the Mid Atlantic, $\$ 11.43$ in 
the South Atlantic, and $\$ 4.65$ in the Gulf of Mexico (NOAA (2010), p. 128). In practice, prices in the US vary across regions based on a number of factors, such as the overall weight and quality of fish (e.g., freshness, fat content, method of storage), the market supply, the consumer demand, the gear used to catch it, and the Japanese Yen/U.S. dollar exchange rate.

Estimating the fishing profit functions, is mainly a matter of retrieving the total harvest cost functions $\left(T C^{A}\right.$ and $\left.T C^{P}\right)$ from the available data. To this end, relationships between Catch Per Unit of Effort (CPUE) and biomass are constructed for both countries, by putting together information from different sources. Figure 5 provides a graphical comparison of 4 different CPUE-Biomass relationships constructed for the US ${ }^{81}$; while Figure 6 depicts 4 such relationships for Canada ${ }^{82}$. Once these 8 relationships were constructed, each of them was solved for effort $\left(E^{A}\right.$ and $\left.E^{P}\right)$. The resulting expressions for effort were then replaced in the harvest cost functions $T C^{A}=\gamma^{A} * E^{A}$, and $T C^{P}=\gamma^{P} * E^{P}$, with $\gamma^{A}$ and $\gamma^{P}$ the unit costs of fishing effort for the two countries. Fishing profit functions for the US (based on $T C^{A}$ ) are reported in Table 2, as specifications K5-K8 (these specifications are derived from the functional relationships

\footnotetext{
${ }^{81}$ Regarding the US biomass, two indices were constructed as weighted sums of Brown's (2007) original SMSM, LGSM and Giant BFT indices: the first, assigned higher weights to the smaller classes $(40 \%, 40 \%$ and $20 \%$ ); the second, equal weights to all classes.

Regarding the US CPUE, ICCAT's on-line Task II c.e. dataset was employed. This dataset gives catches of bluefin by US vessel and by gear type; however, effort (defined as fishing hrs) is not provided by each tuna species. Therefore, two time series for the CPUE were constructed: one, by assuming that the percentage of effort directed specifically to Bluefin tunas was the same as the proportion of Bluefin catches over the total Rod and Reel tuna catches; the other, by assuming that all fishing time was allocated to Bluefin catches.

Using the time series (1993-2007) for the constructed biomass indices and CPUE, the relationships of Figure 5 were derived as follows:

A linear relationship was fitted to the CPUE and biomass index data;

Using a known estimate for the US's biomass in a single year (2007), and rewriting the value of the biomass index (for that year) as "biomass index=biomass*kappa", the value of the parameter kappa was derived using the prediction of the fitted line (for that year);

Applying the value of the parameter kappa across all years, the time series for the biomass index was converted into a time series for biomass;

The CPUE data were plotted against the biomass data (not biomass index), yielding the CPUE-Biomass relationships of Figure 5.

${ }^{82}$ Neilson et al. (2007) was the main source of information for generating these relationships. The relationships of Figure 6 were derived by following steps similar to those in footnote 81 .
} 
of Figure $5^{83}$ ). Fishing profit functions for Canada (based on $T C^{P}$ ) are reported in Table 2, as specifications K1-K4 (derived from the functional relationships of Figure $\left.6^{84}\right)$. For the purposes of the baseline calibrations, the US's $\gamma^{A}$ unit cost of fishing effort is set at $\$ 105$ per fishing hour ${ }^{85}$; and Canada's unit cost of effort is set slightly lower, at $\gamma^{P}=\$ 90$ per hour.

The US derives utility by harvesting the stock and receiving compensation from Canada. For the purposes of the calibration, the square root utility function is employed; this function satisfies the risk averse requirement for the Agent's preferences. The disutility of regulatory effort $c\left(\alpha_{t i}\right)$ is calculated as a percentage (specifically, 9\%) of the US's foregone fishing profits (when exerting regulatory effort $)^{86}$. The US's non-cooperative fishing profits $\pi^{A, N \cdot C \cdot}\left(F_{t}\right)$ are simply her profits without exerting any effort.

The growth of bluefin tuna does not appear to be uniform throughout the year; it is greatest from June through October, when the stock has migrated in large numbers to Canada. During this time, adult fish may increase their body mass by 15-40\%, while feeding on high-energy prey (herring, mackerel and bluefish). For the purposes of the calibration, the growth function $g$ in Canada's area takes the simple form $g\left(S_{\cdot, t}^{A}\right)=1.35 * S_{\cdot, t}^{A}$.

\footnotetext{
${ }^{83}$ Note that $h^{A}$ in Specifications K5-K8 is postmultiplied by 1000 because $h^{A}$ in the profit functions of Table 2 is measured in tons.

${ }^{84}$ Note that $h^{P}$ in Specifications K1-K4 is postmultiplied by 1000 because $h^{P}$ in the profit functions of Table 2 is measured in tons. Moreover, $\gamma^{P}$ in Specifications K1-K4 is postmultiplied by 100 because $\gamma^{P}$ in the profit functions of Table 2 is measured in $\$ / h r$ (while fishing effort in the horizontal axis of Figure 6 was expressed in 100hrs).

${ }^{85} \mathrm{In}$ general, the cost of fishing is a function of the provisions for each trip (hooks, bait, ice, fuel, groceries), the vessel repairs, and the payment to the crew members (NOAA (2008)). Nevertheless, "the available data do not allow the US National Marine Fisheries Service to calculate the effort and cost associated with each trip" (US Federal Register (2010), p. 30737 ). Therefore, the value used in the calibration was constructed based on information for US charter boats in 2004 (NOAA (2010), p. 159).

${ }^{86}$ The disutility of regulatory effort captures the cost of undertaking a conservation policy in the US. The US administration does not announce a budget for enforcing any bluefin tuna regulations; therefore, $c(\alpha)$ is calculated through the opportunity cost of the bluefin tuna fishery. (But even information for the US's budget was available, getting an estimate for $c(\alpha)$ would be complicated: many tuna policies in the US are not directed specifically to bluefin; most tuna permits are of "open access", i.e., allow the landing of all Atlantic tunas (including bluefin), sharks, swordfish and billfish.)
} 
ICCAT (2008) also fits a growth function (to the year-classes after 1970) for the whole Western Atlantic stock. This function yields von Bertalanffy coefficients $K=0.20 ; L \infty=257 ;$ and $t_{0}=0.83 .{ }^{87}$ For the purposes of the calibration, the growth function $F$ in the Adjacent High Sea takes ICCAT's (2008) von Bertalanffy parameters.

For the last 13 years, since ICCAT initiated its 20-year Western Atlantic Rebuilding Plan, the US experienced a shortfall in catches 5 times (2004-2008, and 2010). Therefore, the frequency of shortfalls is about 0.38 . For the purposes of the baseline calibrations, the probability $(1-\theta)$ that the random event occurs is 0.35 .

Finally, the exploitation rate of the stock in the Adjacent High Sea corresponds to Japan's historic quota allocation by ICCAT, i.e., $\lambda=0.17$.

\footnotetext{
${ }^{87}$ Turner and Restrepo's (1994) have also estimated a growth function for the whole stock, with von Bertalanffy coefficients $K=0.08 ; L \infty=382 ; t_{0}=-0.71$.
} 


\subsection{Results of Empirical Calibration}

The parameters and functional relationships of Table 2 are substituted into the static maximization problem of Section 2.3.1. Table 2 contains different Specifications for the fishing profit functions (i.e., Specifications K5-K8 for the US, and K1-K4 for Canada); therefore, maximization is performed for all possible combinations of these Specifications. The results generated by these Specifications, called baseline calibrations, are presented in Tables 3-11 (note that these Tables do not report results for Specification 8 , because Specification 8 is very similar to Specification 7). All results are obtained in Mathematica $7^{88}$.

The way to read Tables 3-11 is the following: the third column of each Table gives the amount of compensation for the levels of the observable output (i.e., for Canada's recruitment). In the simplest setting of the theoretical model (adopted also in the calibration), the observable output takes only three values: low, medium, or high. These values are the result of the interaction of the Agent's regulatory effort with the States of Nature. The Agent, for simplicity, makes a choice between two levels of regulatory effort (first column of Tables 3-11): low effort $\alpha_{1}$, or high effort $\alpha_{2}$. Therefore, the upper half part of Tables 3-11 gives the solutions of the problem in which the Principal (i.e., Canada) induces low effort from the Agent (the US); while the lower half presents the solutions of the problem with high effort. The Principal's expected payoffs, or maximum value functions $V_{1}$ and $V_{2}$, are given in the last column of each Table.

Tables 3-5 report the results of the baseline calibrations when Canada's fishing profit function is given by Specification K5 (for various Specifications of the US's profit function). Instead, Tables 6-8, use Specification K6 for Canada; and Tables 9-11, Specification K7.

\footnotetext{
${ }^{88}$ In order to solve the maximization problem in Mathematica, a linear transformation of the inequality constraints is first required.
} 
The results from all Specifications are qualitatively similar in terms of the maximum value functions: a higher expected payoff is calculated in the high $\left(\alpha_{2}\right)$, than in the low $\left(\alpha_{1}\right)$ effort problem, i.e. $V_{2}>V_{1}$. This means that the Principal is better off by offering a contract which induces high than low effort from the Agent. The estimated level of compensation for the high-effort contract is non decreasing in the medium and high outputs, and the level of compensation is approximately the same in all Tables: $1.38 * 10^{6}$ (Tables $3-5$ ), $1.39 * 10^{6}$ (Tables $6-8$ ), and $1.25 * 10^{6}$ (Tables 9-11). Finding the same values for compensation in Tables 3-8 is not a surprise, because specifications K5 and K6 were constructed based on similar CPUE-Biomass relationships; while specification K7 used a different biomass index. Moreover, Canada's expected payoffs by inducing high effort from the Agent range between $\$$ US5 -10 million $\left(V_{2}\right.$ varies from $5.03 * 10^{6}$ to $10.06 * 10^{6}$, with Specification K7 generating the highest values (Tables 9-11)).

The results of these baseline calibrations were derived under specific model parameterizations. To examine the robustness of the finding $V_{2}>V_{1}$, the maximization problems (for Specifications K5 and K1; K6 and K2; K7 and K4) are repeated for alternate values of parameters: a sensitivity analysis is performed for $\theta$ (i.e., the probability that no random event occurs), the Agent's unit cost of fishing (i.e., parameter $\gamma^{A}$ in Table 2), and the market price of tuna (i.e., parameter $\tau$ in Table 2). The results are drawn in Figures 7-9, 10-12, and 13-15, respectively.

Comparing Figures 7-15, the Principal's maximum value function ${ }^{89}$ varies substantially with the market price of tuna (from $\$ 0.11$ to 13 million); moderately with the probability $\theta$ (less steep curves in Figures 7-9); and remains approximately the same for various levels of the US's unit cost of fishing (in Figure 10, $V_{2}$ varies between $\$ 4.95$ and $\$ 5.03$ million; in Figure 11, it is around

\footnotetext{
${ }^{89} V_{2}$ denotes his expected payoffs when he induces high effort; and $V_{1}$ when he induces low effort.
} 
$\$ 8.4$ million; and in Figure 12, around $\$ 9.9$ million).

Comparing Figures 7-9, the conclusion that the Principal is better off by inducing high (instead of low) effort from the Agent, holds for a large range of $\theta s$. The critical value of $\theta$, above which $V_{2}$ becomes smaller than $V_{1}$, is 0.71 (in Figure 7); 0.86 (in Figure 8), and 0.93 (in Figure 9). It seems safe to argue that for $\theta \leqslant 0.7, V_{2}$ is expected to be larger than $V_{1}$ ( $\theta$ was set at 0.65 in the baseline calibrations of Tables 3-11).

Comparing Figures 10-12, the inequality $V_{2}>V_{1}$ holds again for various values of the Agent's fishing unit cost. Even when the US's fishing cost $\gamma^{A}$ drops down (from the baseline value of $105 \$ / h r$ ) to $5 \$ / h r$, the Principal is still better off by exerting high effort from the Agent (Figures 11 and 12). The critical value, in Figure 10, below which $V_{2}<V_{1}$, is 38. Nevertheless, it is unlikely that the US's fishing unit cost takes such a small value for the US and Canada use similar fishing technology, and the US's parameter $\gamma^{A}$ cannot be significantly different from Canada's value $\gamma^{P}=90$.

Finally, the conclusion $V_{2}>V_{1}$ is quite robust also for changes in the market price of tuna. The inequality is reversed only when the market price falls below $4 \$ / k g$ (Figure 15), or below $9 \$ / k g$ (Figure 14), or below $18 \$ / k g$ (Figure 13). The value in the baseline calibrations of Tables $3-11$ was set at $20 \$ / \mathrm{kg}$.

Overall, the empirical calibration is subject to the usual data limitations and functional specification errors. In this empirical exercise, harvest (production) functions were not properly estimated, and the disutility of effort $\left(c\left(\alpha_{i}\right)\right)$ was chosen with a degree of arbitrariness (for no information regarding the cost of implementing regulations in the bluefin tuna fishery was available). Moreover, constructing fishing profit functions, for either country, involved a significant amount of guesswork (in essence, bringing together different sources of information with a view to to deriving CPUE-Biomass relationships). The main purpose 
of the calibration was to consider the assumptions and limitations of the proposed theoretical model (when trying to project it to a real world setting), and also to raise the use of side payments in the bluefin tuna fishery. 


\section{Concluding Remarks}

My thesis proposes a theoretical framework, in the context of mechanism design, for modeling fisheries agreements with side payments. It also sets up a calibration exercise with parameters inspired by the Western Atlantic Bluefin Tuna fishery.

The theoretical framework examines the extent to which one country (the Agent) responds (i.e., preserves a shared stock) to side payments offered by another country (the Principal). First order conditions for the second best contracts are derived in a static and dynamic setting. The analogue of the Martingale Property, from finance literature, is derived in the context of fisheries.

Though side payments have not received much attention yet, quota transferring is now a policy alternative in the management of the Pacific Salmon, and the Atlantic Tunas. The proposed analysis does not introduce any extra enforcement costs (i.e., other than those already incurring within Regional Fisheries Management Organizations) for the Agent's action remains hidden throughout each fishing season. Moreover, policy makers and fishers may welcome this type of cooperative management more than reciprocal harvesting (the latter is typically viewed as a bribery through which foreigners are granted access into another country's territory). Compensation here, instead, induces the Agent to harvest less extensively, keeping nonetheless her right to exclusively operate in her own area.

In the context of the Western Atlantic Bluefin Tuna, Canada's expected payoffs by proposing this mechanism to the US, range between $\$$ US5-10 million. The results of the calibration should be evaluated keeping in mind the data limitations and functional specification errors. Calibrated models are numerical

models without a complete econometric formulation. Better approximations for the countries' profit and production functions may provide better estimates. 


\section{Appendix A:}

\section{The Optimal Continuation Contract for Two Periods}

In practice, fishing nations renege on agreements and renegotiate over time. Consider the two-period setting of the dynamic contract in Section 2.3.2, but relax the assumption of full commitment: Country A now reneges on the optimal long term contract, in period $t=1$, and exerts effort $\alpha_{1 i} \neq \alpha_{1}^{*}$. Following the realization of $R_{\cdot, \alpha_{1 i}}^{P}$ (at the end of period $t=1$ ), $\mathrm{P}$ offers a new continuation contract to replace the initial long-term contract. The continuation contract has to be mutually beneficial for the two parties.

The optimal continuation contract is a spot contract, i.e., specifies the sec-

ond period compensation schedule $\hat{w_{2}}\left(R_{\cdot, \widehat{\alpha}_{2}}^{P}\right)=\left\{\hat{w_{2}}\left(R_{\theta, \widehat{\alpha}_{2}}^{P}\right), \hat{w_{2}}\left(R_{1-\theta, \widehat{\alpha}_{2}}^{P}\right)\right\}$ as a function of the observable output in that period only. The difference between this contract and the second period spot contract of Section 2.3.2 is the right hand side of the individual rationality constraint. Below, the right hand side of the $(\widehat{I R})$ is written for the optimal values $(*)$ of the contract in Section 2.3.2. The optimal continuation contract with no discounting, and assuming that $\mathrm{P}$ observes $R_{\theta, \alpha_{1 i}}^{P}$ in the end of period $t=1$, is the following:

$$
\max _{w_{2}\left(R_{,, \alpha_{2 i}}^{P}\right)}\left\{\begin{array}{c}
\theta\left\{\pi_{2}^{P}\left(S_{\theta, 2}^{P}, R_{\theta, \alpha_{2 i}}^{P}\right)-w_{2}\left(R_{\theta, \alpha_{2 i}}^{P}\right)\right\} \\
+(1-\theta)\left\{\pi_{2}^{P}\left(S_{1-\theta, 2}^{P}, R_{1-\theta, \alpha_{2 i}}^{P}\right)-w_{2}\left(R_{1-\theta, \alpha_{2 i}}^{P}\right)\right\}
\end{array}\right\}
$$

subject to

the Incentive Compatibility Constraints $(\widehat{I C})$ : 


$$
\begin{gathered}
\left\{\begin{array}{c}
\theta\left\{u\left(\pi_{2}^{A}\left(S_{\theta, 2}^{A}, R_{\theta, 2}^{A}\right)\right)+u\left(w\left(R_{\theta, \alpha_{2 i}}^{P}\right)\right)-c\left(\alpha_{2 i}\right)\right\} \\
+(1-\theta)\left\{u\left(\pi_{2}^{A}\left(S_{1-\theta, 2}^{A}, R_{1-\theta, 2}^{A}\right)\right)+u\left(w\left(R_{1-\theta, \alpha_{2 i}}^{P}\right)\right)-c\left(\alpha_{2 i}\right)\right\}
\end{array}\right\} \\
\geq\left\{\begin{array}{c}
\theta\left\{u\left(\pi_{2}^{A}\left(S_{\theta, 2}^{A}, R_{\theta, 2}^{A}\right)\right)+u\left(w\left(R_{\theta, \alpha_{2 i^{\prime}}}^{P}\right)\right)-c\left(\alpha_{2 i^{\prime}}\right)\right\} \\
+(1-\theta)\left\{u\left(\pi_{2}^{A}\left(S_{1-\theta, 2}^{A}, R_{1-\theta, 2}^{A}\right)\right)+u\left(w\left(R_{1-\theta, \alpha_{2 i^{\prime}}}^{P}\right)\right)-c\left(\alpha_{2 i^{\prime}}\right)\right\}
\end{array}\right\}
\end{gathered}
$$

for all $\alpha_{2 i^{\prime}} \neq \alpha_{2 i}$;

and the Individual Rationality Constraint $(\widehat{I R})$ :

$$
\begin{gathered}
\left\{\begin{array}{c}
\theta\left\{u\left(\pi_{2}^{A}\left(S_{\theta, 2}^{A}, R_{\theta, 2}^{A}\right)\right)+u\left(w\left(R_{\theta, \alpha_{2 i}}^{P}\right)\right)-c\left(\alpha_{2 i}\right)\right\} \\
+(1-\theta)\left\{u\left(\pi_{2}^{A}\left(S_{1-\theta, 2}^{A}, R_{1-\theta, 2}^{A}\right)\right)+u\left(w\left(R_{1-\theta, \alpha_{2 i}}^{P}\right)\right)-c\left(\alpha_{2 i}\right)\right\}
\end{array}\right\} \\
\geq\left\{\begin{array}{c}
\theta\left\{u\left(\pi_{2}^{A}\left(S_{\theta, 2}^{A}, R_{\theta, 2}^{A}\right)\right)+u\left(w_{2}^{*}\left(R_{\theta, \alpha_{2}^{*}}^{P} ; R_{\theta, \alpha_{1}^{*}}^{P}\right)\right)-c\left(\alpha_{2}^{*}\right)\right\} \\
+(1-\theta)\left\{u\left(\pi_{2}^{A}\left(S_{1-\theta, 2}^{A}, R_{1-\theta, 2}^{A}\right)\right)+u\left(w_{2}^{*}\left(R_{1-\theta, \alpha_{2}^{*}}^{P} ; R_{\theta, \alpha_{1}^{*}}^{P}\right)\right)-c\left(\alpha_{2}^{*}\right)\right\}
\end{array}\right\}
\end{gathered}
$$

with:

$$
\begin{aligned}
R_{\theta, 2}^{A} & =\left[F\left((1-\lambda)\left(R_{\theta, \alpha_{1 i}}^{P}-h_{1}^{P}\right)\right)\right]_{\theta, 2} \\
R_{1-\theta, 2}^{A} & =\left[F\left((1-\lambda)\left(R_{\theta, \alpha_{1 i}}^{P}-h_{1}^{P}\right)\right)\right]_{1-\theta, 2}
\end{aligned}
$$




\section{Appendix B:}

\section{The Infinitely Repeated Contract}

So far, the relation between the contracting parties has been finite. Appendix $\mathrm{B}$ relaxes this assumption. The repeated nature of the transaction makes the agreement below Self-Enforcing.

Some intuition for capturing the Self-Enforcing nature of the agreement is drawn by the Relational Contracts literature (Telser (1980), Klein and Leffler (1981), Bull (1987), MacLeod and Malcomson (1989), MacLeod (2003, 2007), Baker (1992) and Baker et al. (1994, 2002)). In this literature, a Self-Enforcing employment contract is sustained by the use of credible threats. The reason why threats are credible, is that the contracting parties are expected to interact infinitely over time. Indeed, in fisheries, the transaction is a repeated one; fish reproduce over time.

Based on the literature on relational contracts under asymmetric information, the optimal Self Enforcing contract is a perfect Bayesian Equilibrium of the infinite horizon game. In a perfect Bayesian equilibrium, the parties' strategies (A's strategy is her action $\alpha_{t}$; P's strategy is the recruitment-contingent compensation $w_{t}$ ), following any history of output realizations and any history of past payments, are best responses. MacLeod and Malcomson (1989) have shown that non-stationary allocations cannot improve on stationary ones. This means that an efficient incentive compatible allocation involves A taking a constant action over time, and $\mathrm{P}$ paying a constant compensation over time.

In the setting of Section 2.1, the optimal stationary contract maximizes the joint surplus from the agreement, and is the solution of the following dynamic programming problem ${ }^{90}$ :

${ }^{90}$ The problem is now written with a continuous level of effort $\alpha$. 


$$
s^{* *}=\max _{w\left(R_{\theta}^{P}\right), w\left(R_{1-\theta}^{P}\right), \alpha}\left\{(1-\delta) E_{R_{\cdot}^{P}}\left[\begin{array}{c}
\pi^{P}\left(S_{\cdot}^{P}, R_{\cdot}^{P}\right)-w\left(R_{\cdot}^{P}\right)+ \\
u\left(\pi^{A}\left(S_{\cdot}^{A}, R_{\cdot}^{A}\right)\right)+u\left(w\left(R_{\cdot}^{P}\right)\right)-c(\alpha) \mid \alpha \\
+\delta E_{R^{P}}\left[s^{* *} \mid \alpha^{* *}\right]
\end{array}\right\}\right.
$$

subject to

the Incentive Compatibility Constraint $\left(I C^{* *}\right)$

$$
\alpha^{* *} \in \underset{\widetilde{\alpha}}{\arg \max }\left\{\left[\begin{array}{c}
\theta\left\{u\left(\pi^{A}\left(S_{\theta}^{A}, R_{\theta}^{A}\right)\right)+u\left(w\left(R_{\theta}^{P}\right)\right)-c(\widetilde{\alpha})\right\} \\
+(1-\theta)\left\{u\left(\pi^{A}\left(S_{1-\theta}^{A}, R_{1-\theta}^{A}\right)\right)+u\left(w\left(R_{1-\theta}^{P}\right)\right)-c(\widetilde{\alpha})\right\} \\
+\frac{\delta}{1-\delta} s^{A * *}
\end{array}\right]\right\}
$$

and the two Individual Rationality Constraints $\left(I R A^{* *}\right.$ for $\mathrm{A} ; I R P^{* *}$ for $\left.\mathrm{P}\right)$

$$
\begin{gathered}
\left.I C^{* *}\right|_{\alpha^{* *}} \geq \frac{\delta}{1-\delta} \bar{s}^{A} \\
{\left[\begin{array}{c}
\left\{\theta \pi^{P}\left(S_{\theta}^{P}, R_{\theta}^{P}\right)+(1-\theta) \pi^{P}\left(S_{1-\theta}^{P}, R_{1-\theta}^{P}\right)\right\} \\
-\left\{\theta w\left(R_{\theta}^{P}\right)+(1-\theta) w\left(R_{1-\theta}^{P}\right)\right\} \\
+\frac{\delta}{1-\delta}\left(s^{* *}-s^{A * *}\right)
\end{array}\right] \geq \frac{\delta}{1-\delta} \bar{s}^{P}}
\end{gathered}
$$

with $\delta$ denoting the common discount factor; $E$ the expectation operator (with respect to $R_{.}^{P}$ ); $s^{* *}$ the joint surplus from the optimal contract in some time period; $s^{A * *}$ A's share of the joint surplus $s^{* *}$ (thus, $\left(s^{* *}-s^{A * *}\right)$ denotes P's share); $\bar{s}^{A}$ and $\bar{s}^{P}$ A's and P's non-cooperative payoffs respectively. The solution of this model will depend on the non-cooperative payoffs $\bar{s}^{A}$ and $\bar{s}^{P}$ (determined from the non-cooperative equilibria of Section 2.2). 
Figure 1: The Stochastic Sequential Fishery

\section{A's EEZ}

P's EEZ

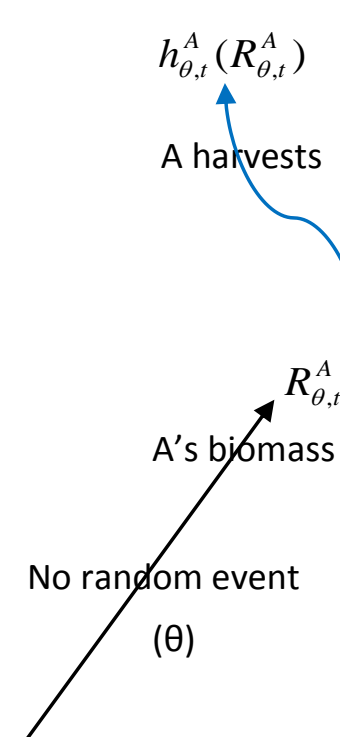

A

Biomass $R_{\bullet}, t$

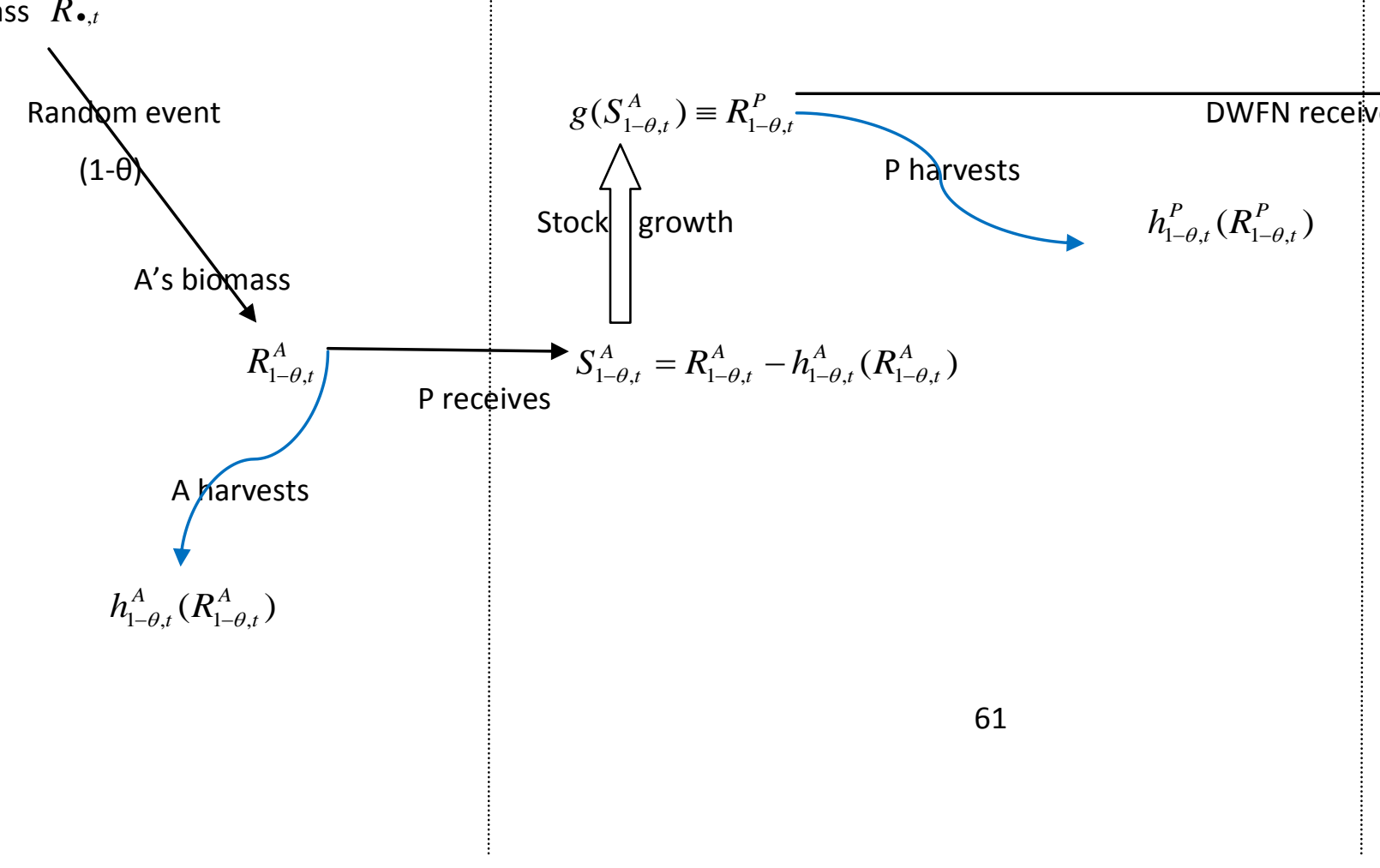

\section{AHS}

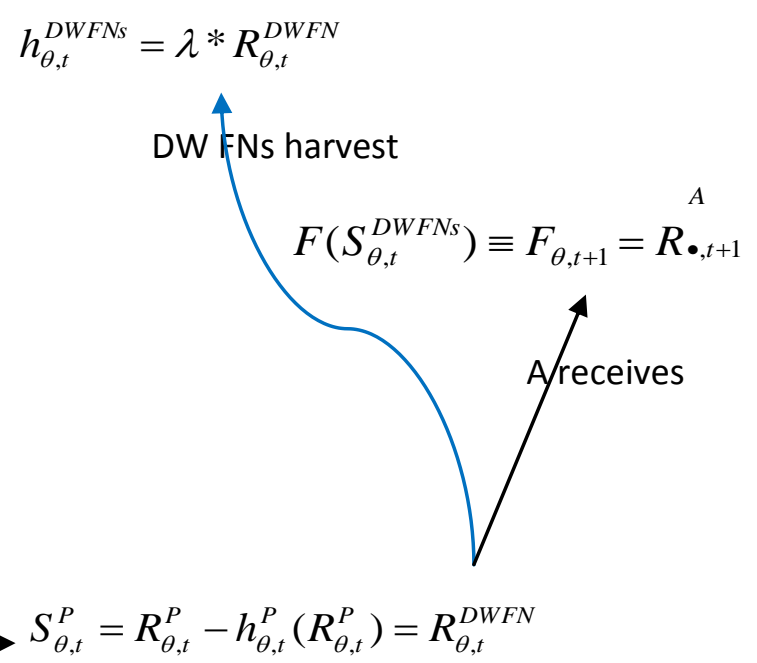

DWFN receive $S_{\theta, t}^{P}=R_{\theta, t}^{P}-h_{\theta, t}^{P}\left(R_{\theta, t}^{P}\right)=R_{\theta, t}^{D W F N}$ 
Figure 2: Side Payments in the Stochastic Sequential Fishery

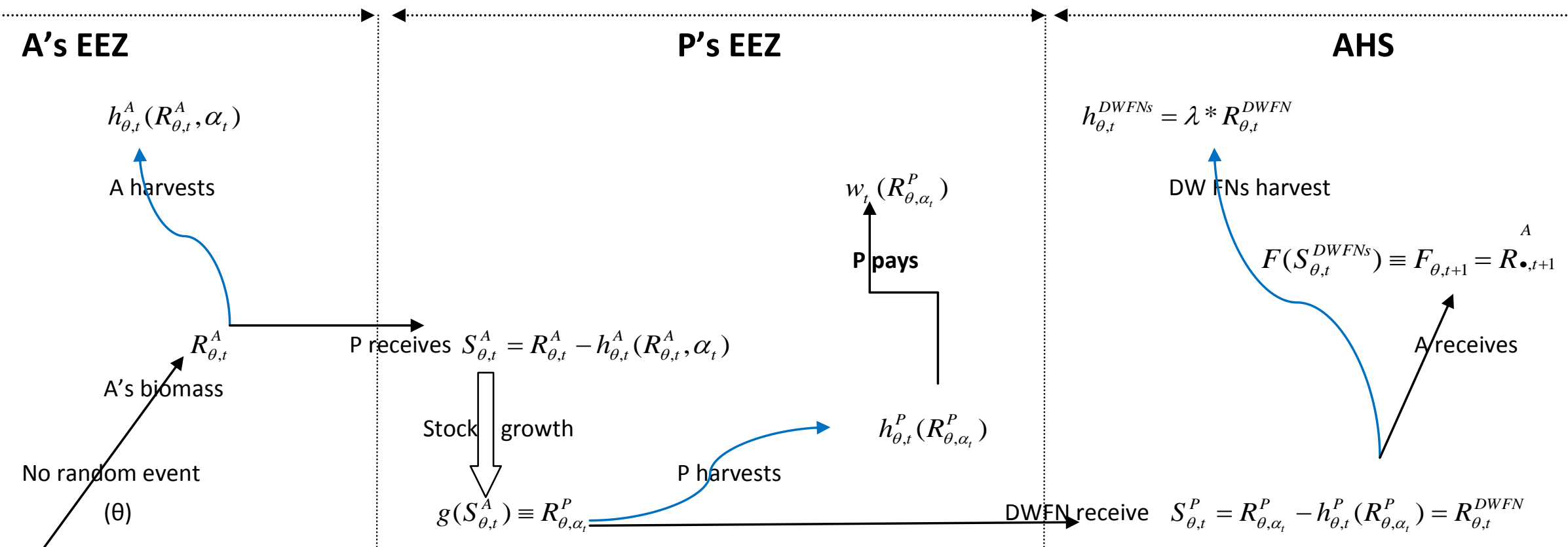

Biomass $R \bullet$

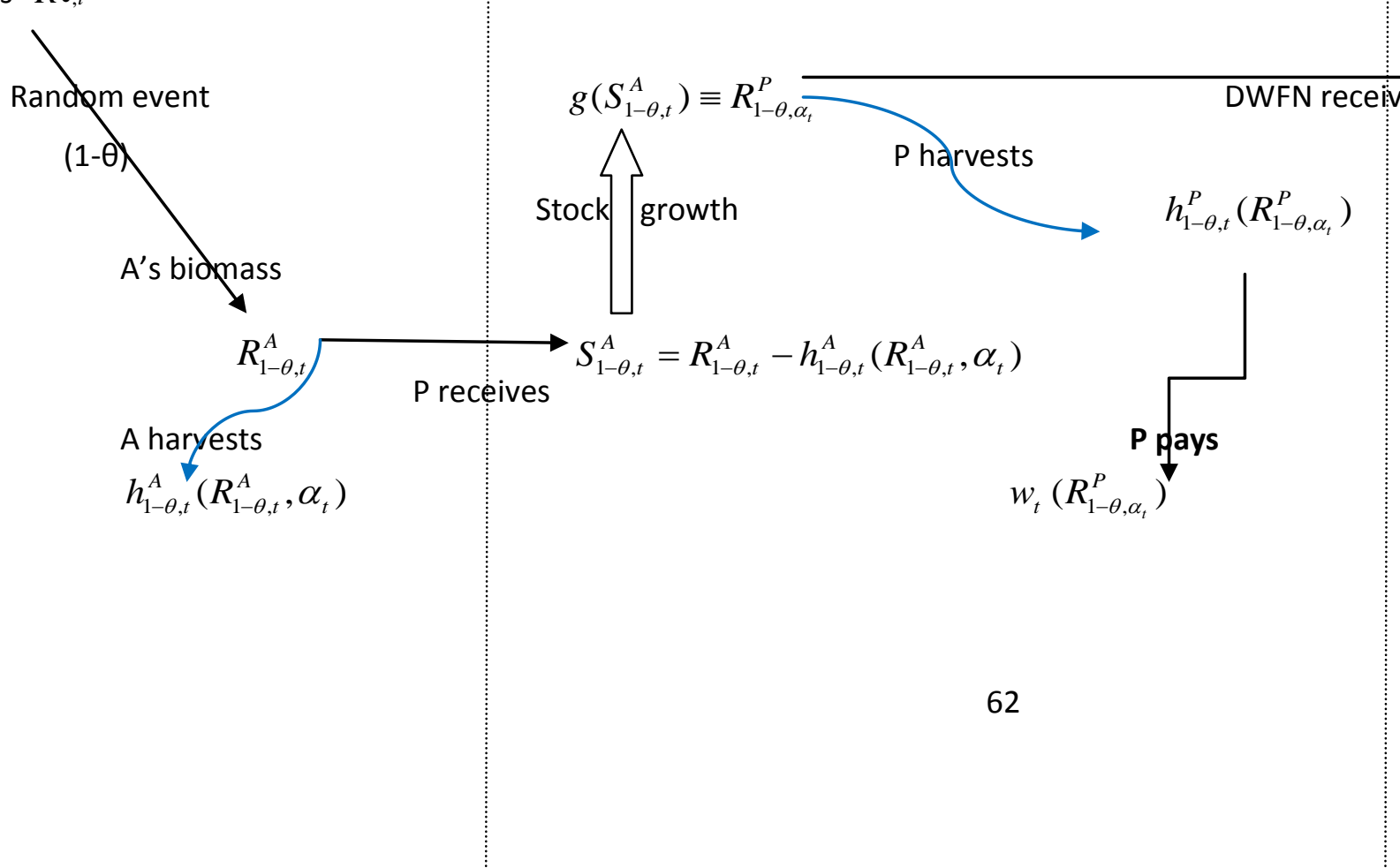


Figure 3: ICCAT's Convention Area for Bluefin Tuna (separated in West and East Management Area by the $45^{\circ}$ West meridian)

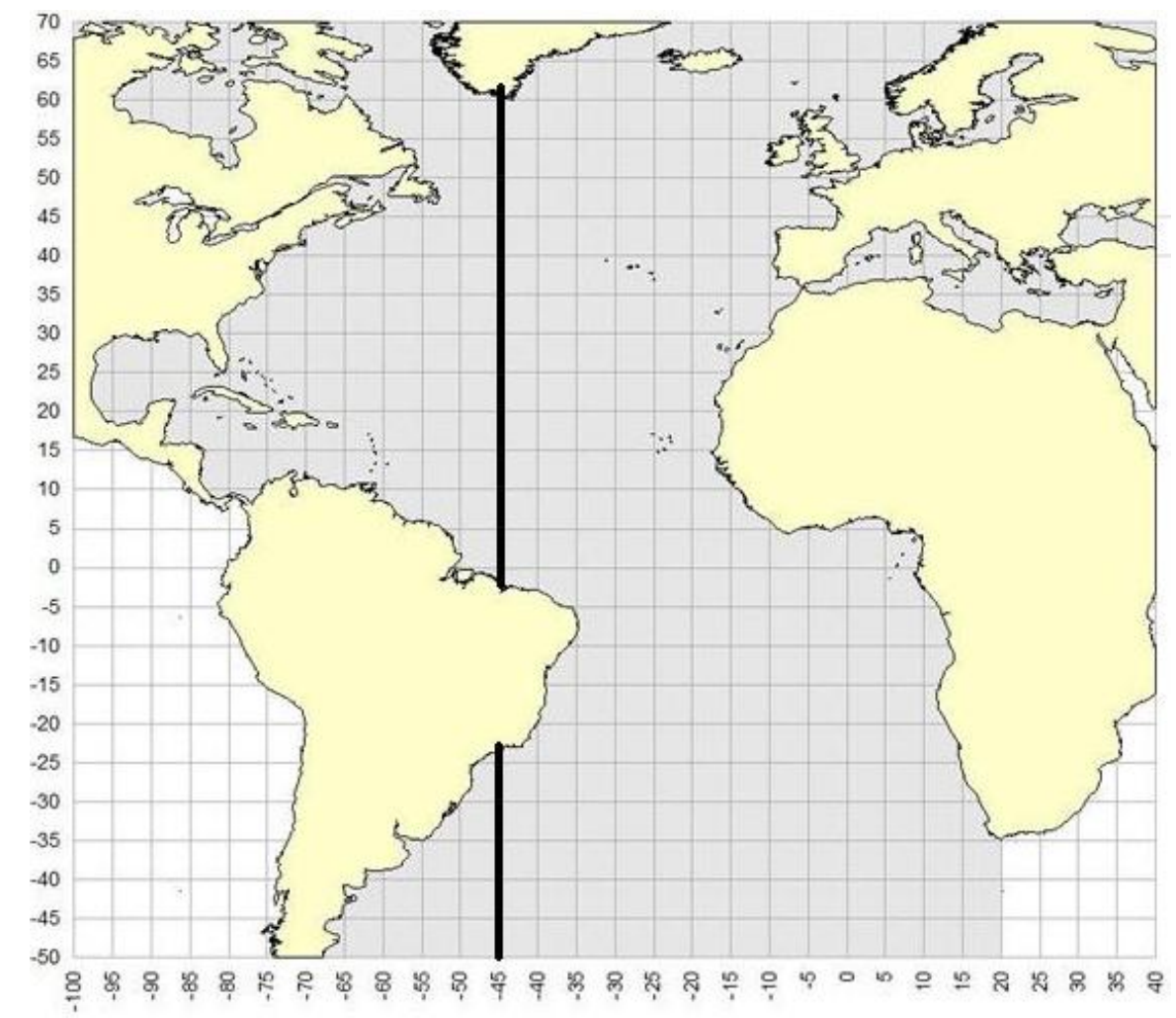

Figure 4: Historical Catches of Western Atlantic Bluefin Tuna (ATW) in comparison to Total Allowable Catches (TAC) set by ICCAT

(Source: ICCAT (20010))

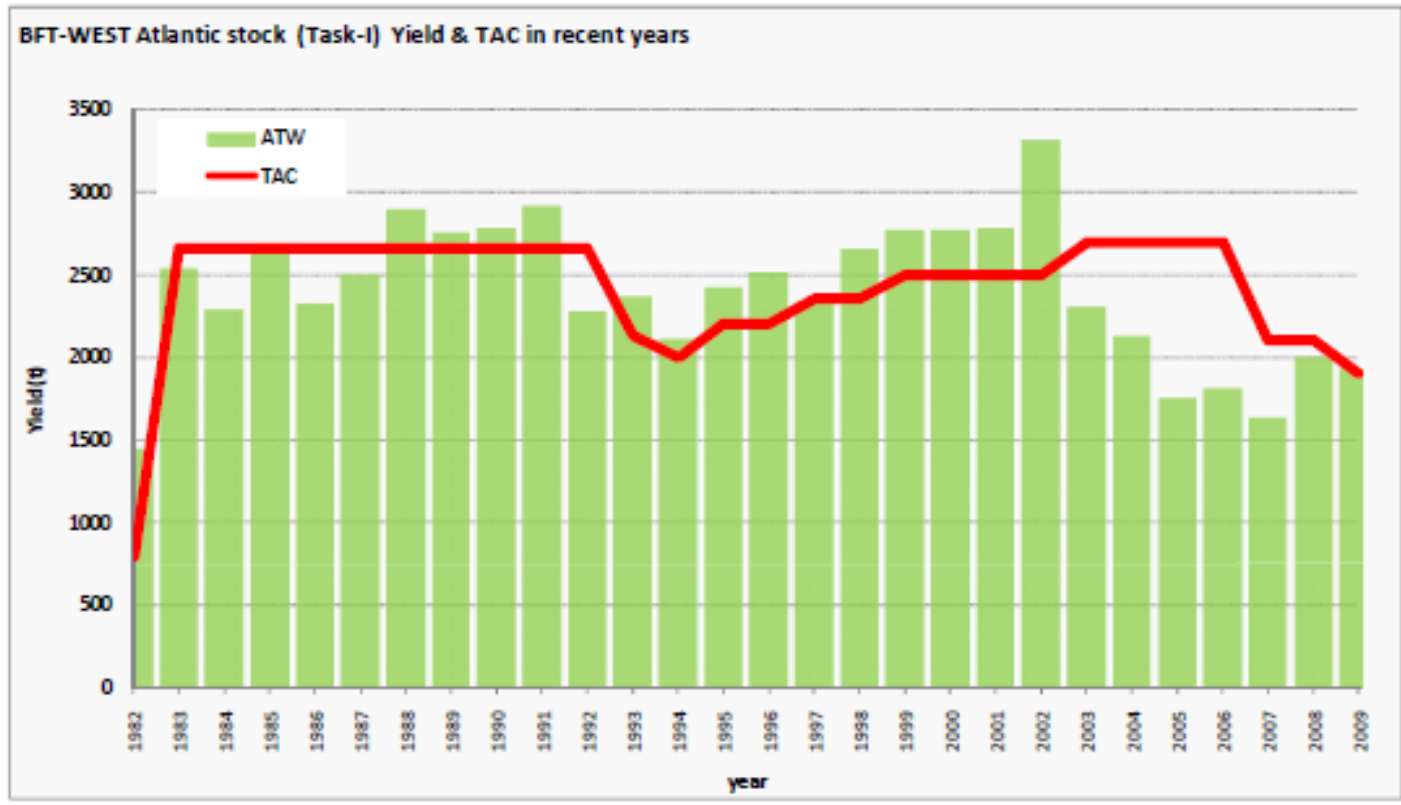


Figure 5: CPUE-Biomass Relationships for the US Bluefin Tuna fishery

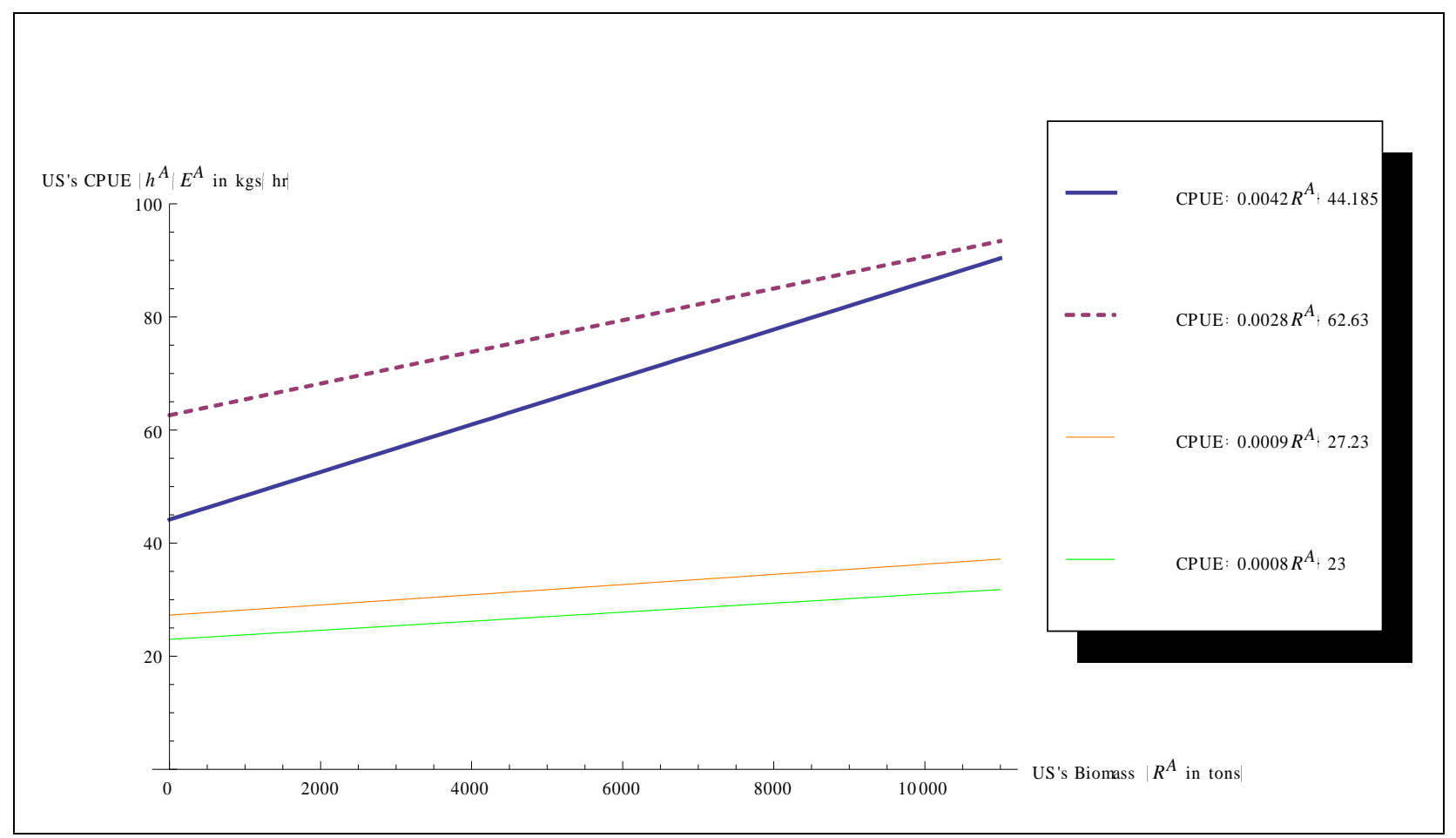

Figure 6: CPUE-Biomass Relationships for the Canadian Bluefin Tuna fishery

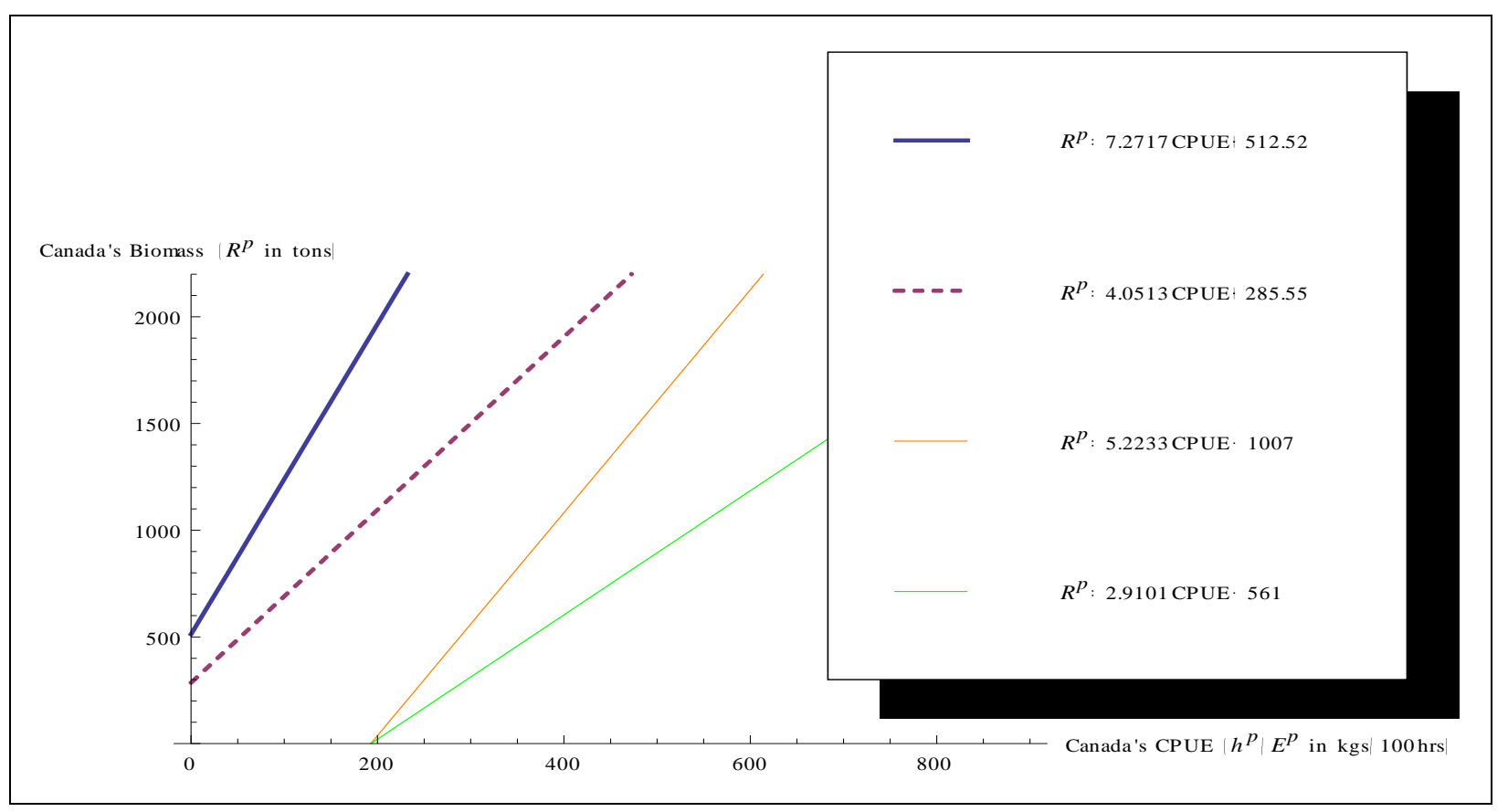


Figure 7: Sensitivity Analysis: K5 and K1 Specifications for alternate values of $\theta$

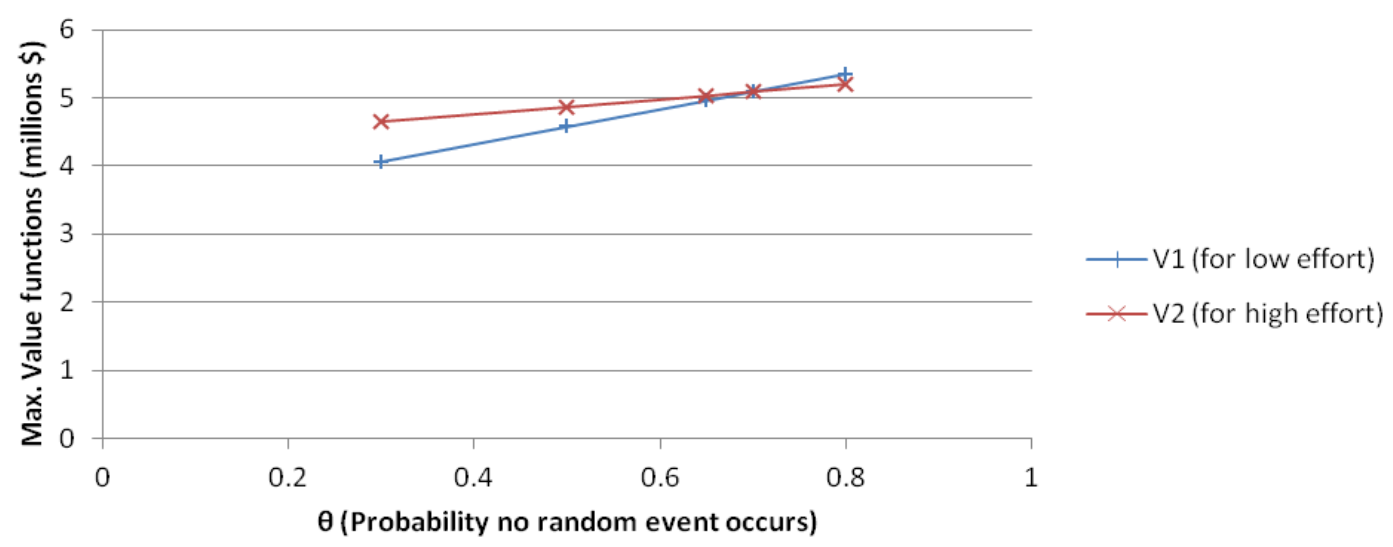

Figure 8: Sensitivity Analysis: K6 and K2 Specifications for alternate values of $\theta$

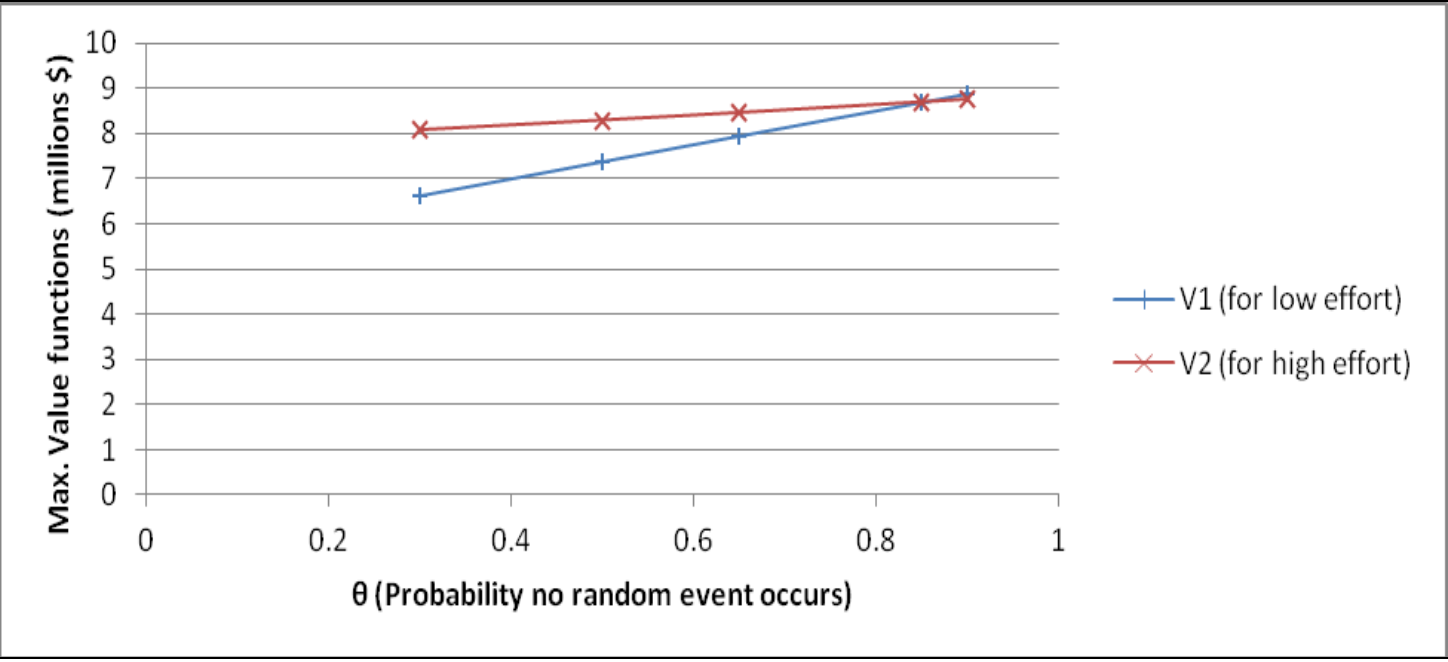

Figure 9: Sensitivity Analysis: K7 and K4 Specifications for alternate values of $\theta$

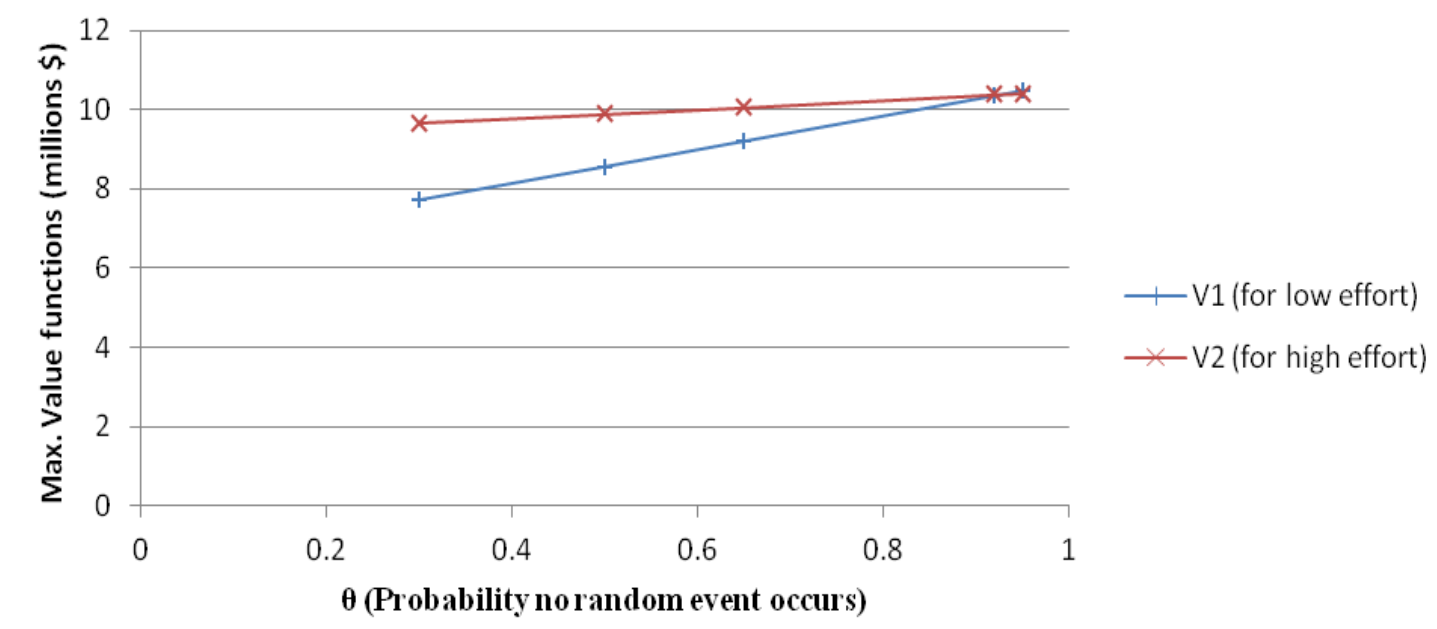


Figure 10: Sensitivity Analysis: K5 and K1 Specifications for alternate values of the US's fishing unit cost

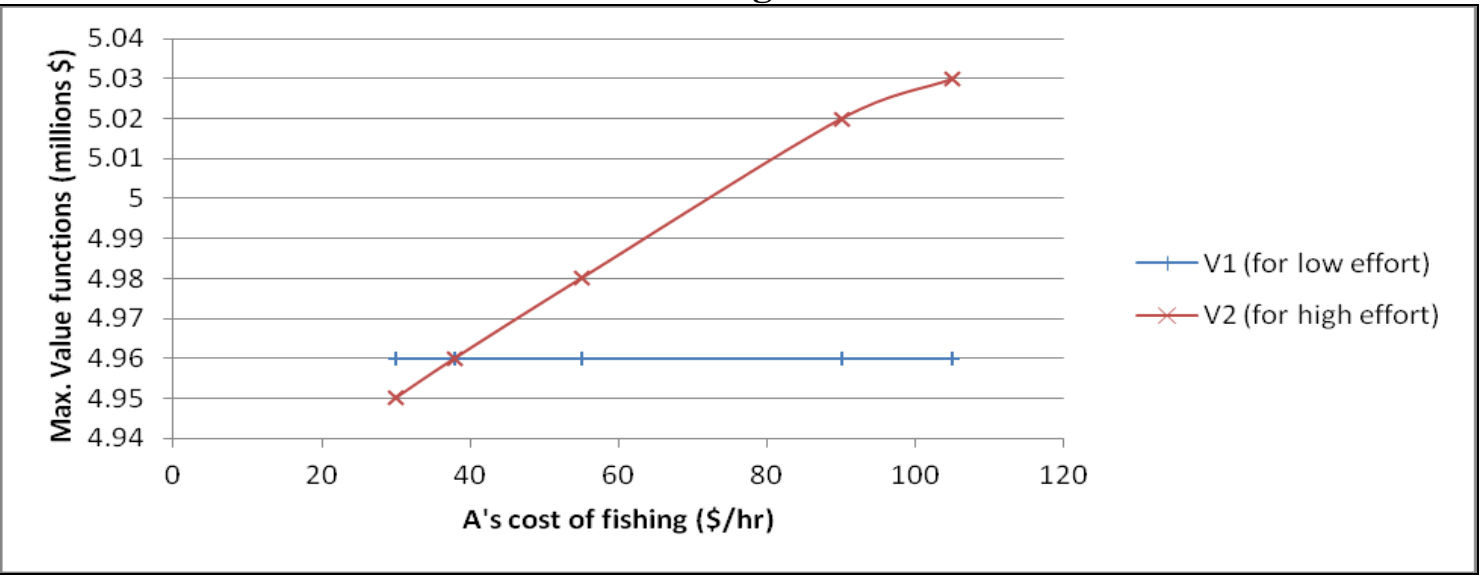

Figure 11: Sensitivity Analysis: K6 and K2 Specifications for alternate values of the US's fishing unit cost

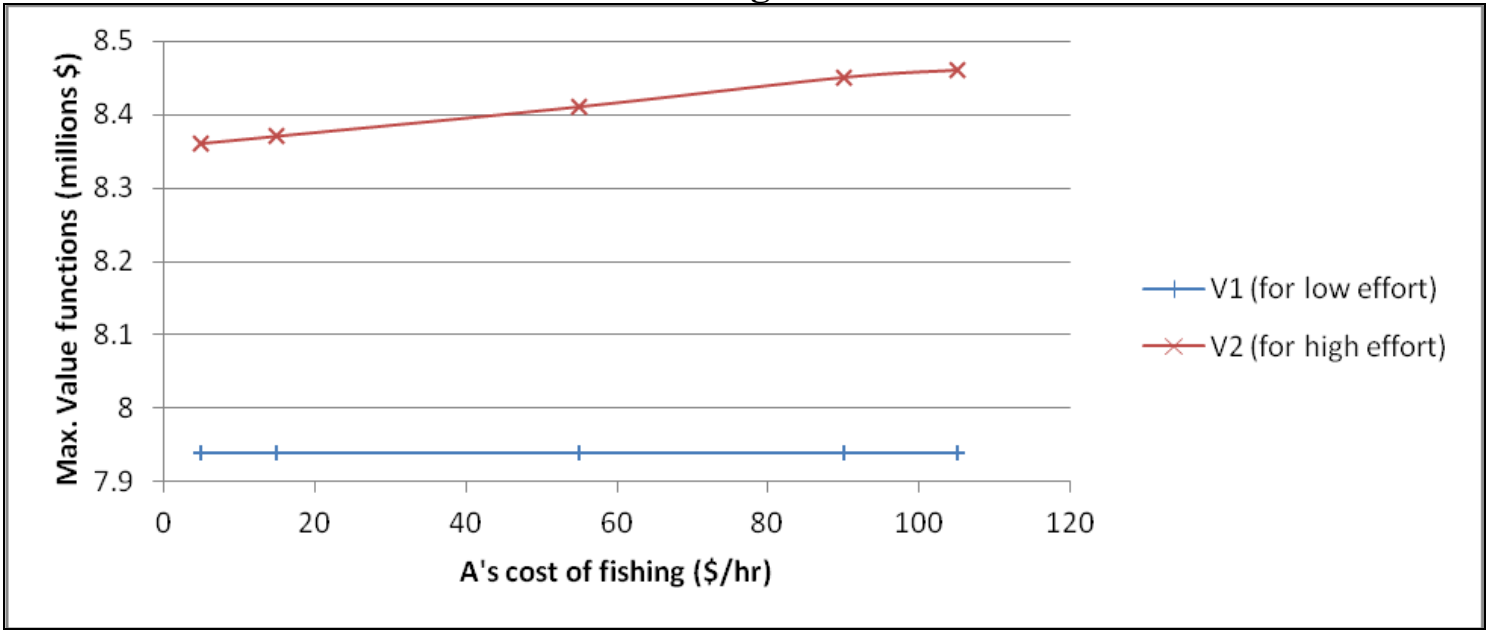

Figure 12: Sensitivity Analysis: K7 and K4 Specifications for alternate values of the US's fishing unit cost

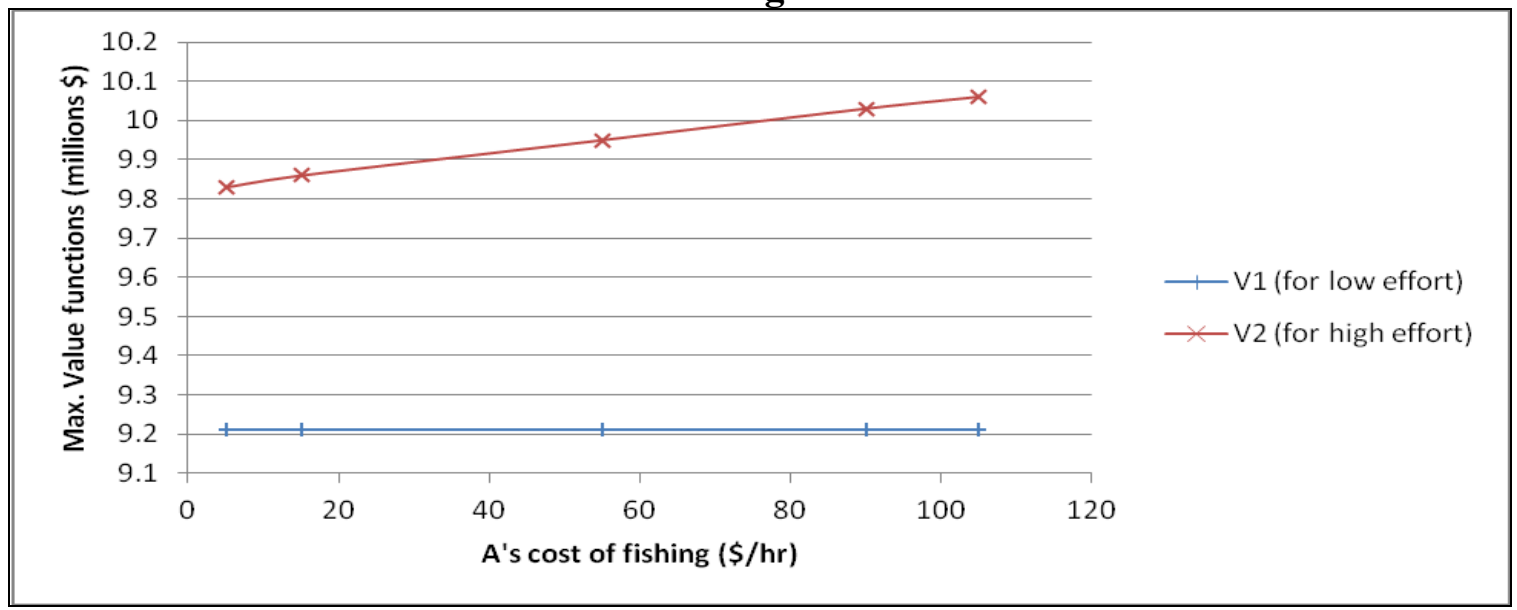


Figure 13: Sensitivity Analysis: K5 and K1 Specifications for alternate values of tuna's market price

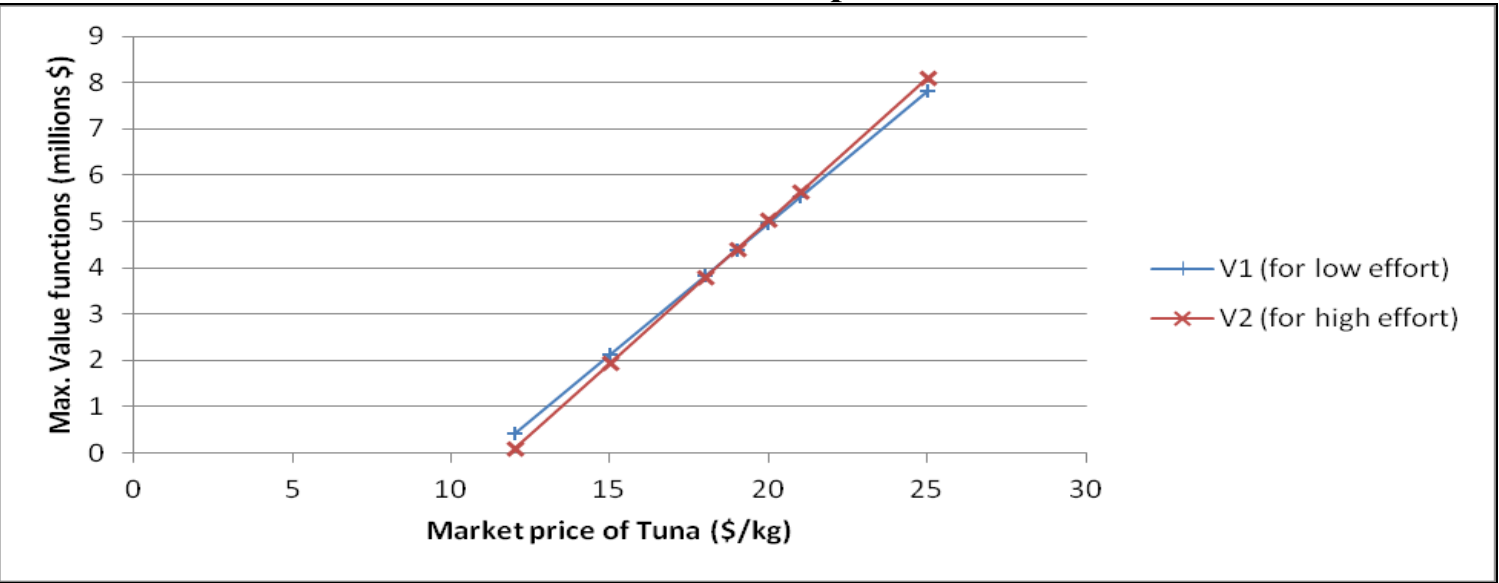

Figure 14: Sensitivity Analysis: K6 and K2 Specifications for alternate values of tuna's market price

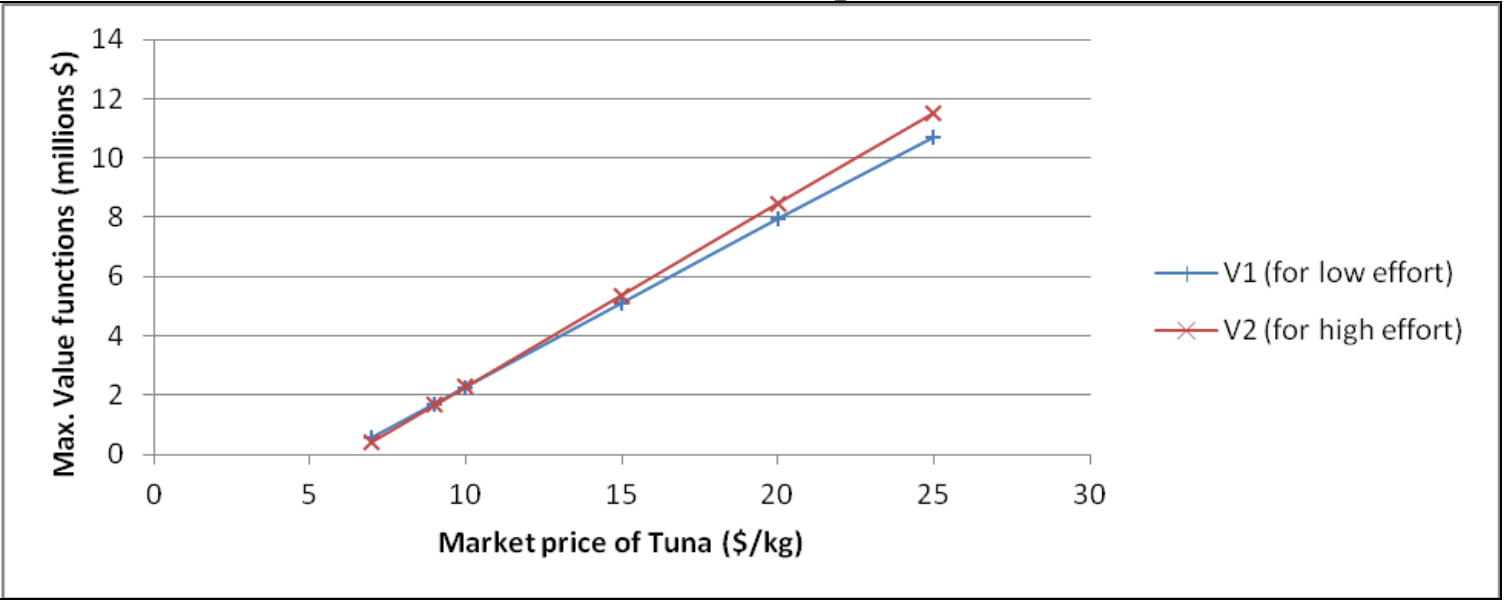

Figure 15: Sensitivity Analysis: K7 and K4 Specifications for alternate values of tuna's market price

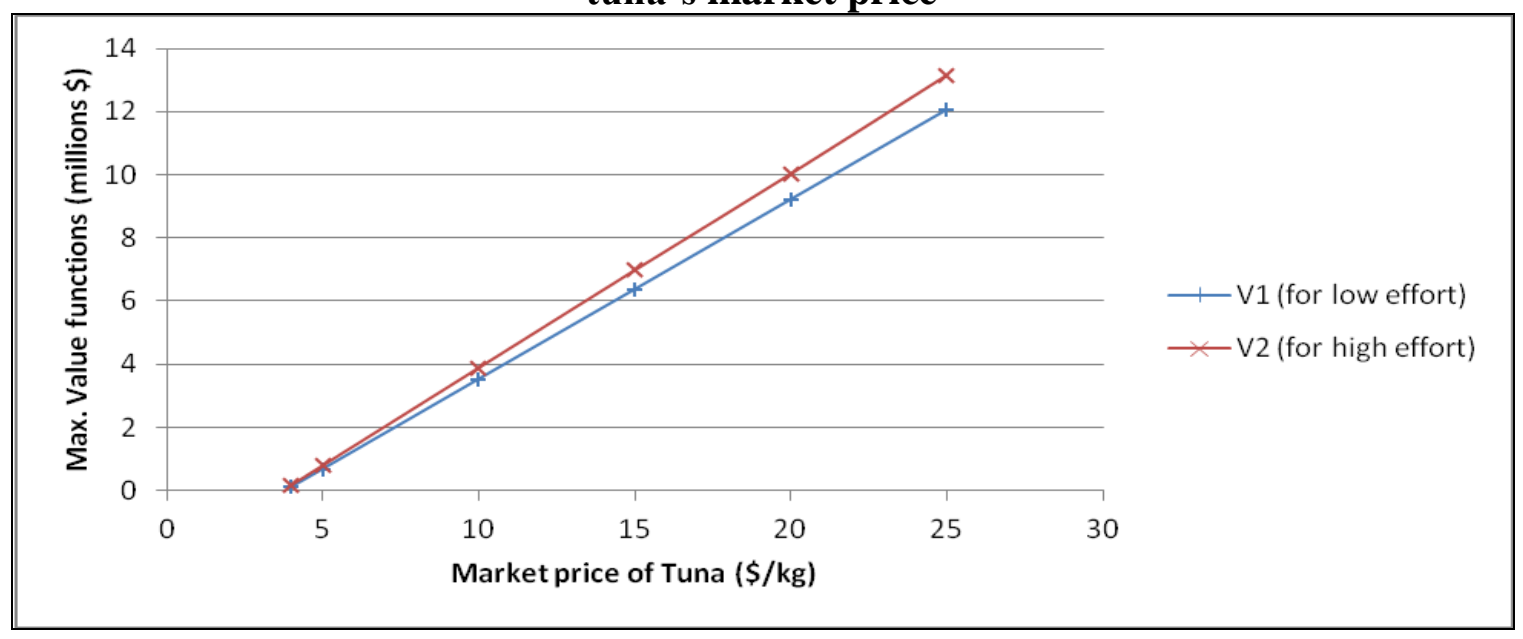


Table 1: Main International Fisheries Organizations by type of policy arrangement (Abundance-based catch limits, or Reciprocal harvesting)

\begin{tabular}{|c|c|c|}
\hline & Abundance-based catch limits & Reciprocal Harvesting \\
\hline \multirow[t]{14}{*}{$\begin{array}{l}\text { Atlantic } \\
\text { Ocean }\end{array}$} & $\begin{array}{c}\text { International Commission for the } \\
\text { Conservation of the Atlantic } \\
\text { Tunas }\end{array}$ & $\begin{array}{l}1997 \text { EU-Faroe Islands Atlanto- } \\
\text { Scandian Herring Agreement }\end{array}$ \\
\hline & $\begin{array}{l}\text { North Atlantic Salmon } \\
\text { Conservation Organization }\end{array}$ & $\begin{array}{l}1998 \text { EU-Mozambique Tuna } \\
\text { Agreement }\end{array}$ \\
\hline & $\begin{array}{l}\text { North Atlantic Fishery } \\
\text { Organization } \\
\end{array}$ & $\begin{array}{c}2001 \text { EU-Mauritania Cephalopods and } \\
\text { Tuna Agreement }\end{array}$ \\
\hline & $\begin{array}{l}\text { 2006 EU-Norway North Sea } \\
\text { Stocks Agreement (Cod, }\end{array}$ & $\begin{array}{l}2004 \text { EU-Guinea Cephalopods and } \\
\text { Shrimp Agreement }\end{array}$ \\
\hline & Haddock, Saithe, Place, Mackerel, & 2004 EU-Madagascar Tuna Agreement \\
\hline & Herring) & 2005 EU-Cape Verde Tuna Agreement \\
\hline & & 2005 EU-Gabon Tuna Agreement \\
\hline & & 2005 EU-Morocco Tuna Agreement \\
\hline & & $\begin{array}{c}2005 \text { EU-Norway Blue Whiting } \\
\text { Agreement }\end{array}$ \\
\hline & & $\begin{array}{l}2006 \text { EU-Norway Spring-spawning } \\
\text { Herring Agreement }\end{array}$ \\
\hline & & $\begin{array}{c}2006 \text { EU-Russia Baltic Sea Stocks } \\
\text { Agreement (Cod, Salmon, Sprat, } \\
\text { Herring) }\end{array}$ \\
\hline & & $\begin{array}{l}2007 \text { EU-Faroe Islands Blue Whiting } \\
\text { Agreement }\end{array}$ \\
\hline & & $\begin{array}{l}2007 \text { EU-Greenland Halibut, Shrimp, } \\
\text { Redfish Agreement }\end{array}$ \\
\hline & & 2007 EU-Ivory Coast Tuna Agreement \\
\hline \multirow[t]{6}{*}{$\begin{array}{l}\text { Pacific } \\
\text { Ocean }\end{array}$} & $\begin{array}{l}\text { Inter-American Tropical Tuna } \\
\text { Commission }\end{array}$ & $\begin{array}{c}2002 \text { US-Canada Pacific Albacore } \\
\text { Tuna Agreement }\end{array}$ \\
\hline & $\begin{array}{l}\text { International Pacific Halibut } \\
\text { Commission }\end{array}$ & $\begin{array}{l}2003 \text { US-Pacific Islands Tuna } \\
\text { Agreement }\end{array}$ \\
\hline & $\begin{array}{l}\text { North Pacific Anadromous Fish } \\
\text { Commission }\end{array}$ & \\
\hline & Pacific Salmon Commission & \\
\hline & $\begin{array}{l}\text { Commission for the Conservation } \\
\text { of the Southern Bluefin Tuna }\end{array}$ & \\
\hline & $\begin{array}{l}2003 \text { US-Canada Pacific Hake } \\
\text { (Whiting) Agreement }\end{array}$ & \\
\hline $\begin{array}{c}\text { Southern } \\
\text { Antarctic } \\
\text { Ocean }\end{array}$ & $\begin{array}{c}\text { Commission for the Conservation } \\
\text { of the Antarctic Marine Living } \\
\text { Resources }\end{array}$ & \\
\hline
\end{tabular}


Table 2: Summary of Parameters and Functional Relationships for the Empirical Baseline Calibrations (US: Agent (A); Canada: Principal (P))

\begin{tabular}{|c|c|}
\hline $\begin{array}{l}\text { Component of } \\
\text { Theory }\end{array}$ & Empirical Value for Calibration \\
\hline $\begin{array}{l}\text { Initial stock } \\
\text { biomass }\end{array}$ & $F_{t+1}=5940$ tons \\
\hline A's recruitment & $\begin{array}{l}R_{\theta, t}^{A}=5940 \text { tons } \\
R_{1-\theta, t}^{A}=5346 \text { tons }\end{array}$ \\
\hline A's harvest function & $\begin{array}{l}h_{\theta, t}^{A}\left(R_{\theta, t}^{A}, \alpha_{t 1}\right)=1200 \text { tons } \\
h_{1-\theta, t}^{A}\left(R_{1-\theta, t}^{A}, \alpha_{t 1}\right)=840 \text { tons } \\
h_{\theta, t}^{A}\left(R_{\theta, t}^{A}, \alpha_{t 2}\right)=960 \text { tons } \\
h_{1-\theta, t}^{A}\left(R_{1-\theta, t}^{A}, \alpha_{t 2}\right)=660 \text { tons }\end{array}$ \\
\hline $\begin{array}{l}\text { A's fishing profit } \\
\text { function }\end{array}$ & $\begin{array}{l}\pi^{A}\left(h^{A}, R^{A}\right)=\tau * h^{A} * 1000-\frac{\gamma^{A} * h^{A} * 1000}{0.0042 * R^{A}+44.185} \rightarrow \mathrm{K} 5 \text { specification } \\
\pi^{A}\left(h^{A}, R^{A}\right)=\tau * h^{A} * 1000-\frac{\gamma^{A} * h^{A} * 1000}{0.0028 * R^{A}+62.63} \rightarrow \mathrm{K} 6 \text { specification } \\
\pi^{A}\left(h^{A}, R^{A}\right)=\tau * h^{A} * 1000-\frac{\gamma^{A} * h^{A} * 1000}{0.0009 * R^{A}+27.23} \rightarrow \mathrm{K} 7 \text { specification } \\
\pi^{A}\left(h^{A}, R^{A}\right)=\tau * h^{A} * 1000-\frac{\gamma^{A} * h^{A} * 1000}{0.0008 * R^{A}+23} \rightarrow \mathrm{K} 8 \text { specification } \\
\tau=20 \frac{\$}{k g} \\
\gamma^{A}=105 \frac{\$}{h r}\end{array}$ \\
\hline A's utility & Square root function \\
\hline A's disutility & $c\left(\alpha_{t i}\right)=0.09 *\left(\right.$ foregone $_{-}$profits $)$ \\
\hline $\begin{array}{l}\text { A's non cooperative } \\
\text { fishing profits }\end{array}$ & $\pi^{A, N . C .}\left(F_{t}\right)=$ foregone_profits \\
\hline $\begin{array}{l}\text { Stock's growth } \\
\text { function in P's area }\end{array}$ & $g\left(S^{A}\right)=1.35 * S^{A}$ \\
\hline P's harvest function & $\begin{array}{l}h_{1-\theta, t}^{P}\left(R_{1-\theta, t}^{P}, \alpha_{t 1}\right)=400 \text { tons } \\
h_{\theta, t}^{P}\left(R_{\theta, t}^{P}, \alpha_{t 1}\right)=660 \text { tons } \\
h_{1-\theta, t}^{P}\left(R_{1-\theta, t}^{P}, \alpha_{t 2}\right)=660 \text { tons } \\
h_{\theta, t}^{P}\left(R_{\theta, t}^{P}, \alpha_{t 2}\right)=710 \text { tons }\end{array}$ \\
\hline
\end{tabular}




\begin{tabular}{|c|c|}
\hline $\begin{array}{l}\text { P's fishing profit } \\
\text { function }\end{array}$ & $\begin{array}{l}\pi^{P}\left(h^{P}, R^{P}\right)=\tau * h^{P} * 1000-\frac{\gamma^{P} * 100 * 7.2717 * h^{P} * 1000}{R^{P}-512.52} \rightarrow \mathrm{K} 1 \\
\text { specification } \\
\pi^{P}\left(h^{P}, R^{P}\right)=\tau * h^{P} * 1000-\frac{\gamma^{P} * 100 * 4.051 * h^{P} * 1000}{R^{P}-285.55} \rightarrow \mathrm{K} 2 \\
\text { specification } \\
\pi^{P}\left(h^{P}, R^{P}\right)=\tau * h^{P} * 1000-\frac{\gamma^{P} * 100 * 5.2233 * h^{P} * 1000}{R^{P}+1007} \rightarrow \mathrm{K} 3 \\
\text { specification } \\
\pi^{P}\left(h^{P}, R^{P}\right)=\tau * h^{P} * 1000-\frac{\gamma^{P} * 100 * 2.9101 * h^{P} * 1000}{R^{P}+561} \rightarrow \mathrm{K} 4 \\
\text { specification } \\
\tau=20 \frac{\$}{k g} \\
\gamma^{P}=90 \frac{\$}{h r}\end{array}$ \\
\hline $\begin{array}{l}\text { Probability of } \\
\text { random event }\end{array}$ & $1-\theta=0.35$ \\
\hline $\begin{array}{l}\text { Exploitation rate in } \\
\text { the AHS }\end{array}$ & $\lambda=0.17$ \\
\hline $\begin{array}{l}\text { Stock's growth } \\
\text { function in the AHS }\end{array}$ & $K=0.2 ; L_{\infty}=257 ; t_{0}=0.83$ \\
\hline
\end{tabular}


Table 3: Results of baseline calibration with K5 and K1 Specifications

\begin{tabular}{|c|c|c|c|}
\hline A's effort & Observable Output & Compensation (\$) & $\begin{array}{l}\text { Maximum Value } \\
\text { Function (\$) }\end{array}$ \\
\hline \multirow{3}{*}{ Low $\left(\alpha_{1}\right)$} & Low & 236 & \multirow{3}{*}{$V_{1}=4.96 * 10^{6}$} \\
\hline & Medium & 236 & \\
\hline & High & 317 & \\
\hline \multirow[b]{2}{*}{$\operatorname{High}\left(\alpha_{2}\right)$} & Low & $6.7 * 10^{7}$ & \multirow[b]{2}{*}{$V_{2}=5.03 * 10^{6}$} \\
\hline & Medium & $1.38 * 10^{6}$ & \\
\hline
\end{tabular}

Table 4: Results of baseline calibration with K5 and K2 Specifications

\begin{tabular}{|l|l|l|l|}
\hline A's effort & Observable Output & Compensation (\$) & $\begin{array}{l}\text { Maximum Value } \\
\text { Function (\$) }\end{array}$ \\
\hline \multirow{3}{*}{ Low $\left(\alpha_{1}\right)$} & Low & 236 & \multirow{2}{*}{$V_{1}=7.94 * 10^{6}$} \\
\cline { 2 - 3 } & Medium & 236 & \\
\cline { 2 - 3 } & High & 317 & \multirow{2}{*}{$V_{2}=8.47 * 10^{6}$} \\
\hline \multirow{3}{*}{ High $\left(\alpha_{2}\right)$} & Low & $6.1^{*} 10^{6}$ & \\
\cline { 2 - 3 } & Medium & $1.38^{*} 10^{6}$ & \\
\cline { 2 - 3 } & High & $1.38^{*} 10^{6}$ & \\
\hline
\end{tabular}

Table 5: Results of baseline calibration with K5 and K3 Specifications

\begin{tabular}{|c|c|c|c|}
\hline A's effort & Observable Output & Compensation (\$) & $\begin{array}{l}\text { Maximum Value } \\
\text { Function (\$) }\end{array}$ \\
\hline \multirow{3}{*}{ Low $\left(\alpha_{1}\right)$} & Low & 236 & \multirow{3}{*}{$V_{1}=7.72 * 10^{6}$} \\
\hline & Medium & 236 & \\
\hline & High & 317 & \\
\hline \multirow[b]{2}{*}{$\operatorname{High}\left(\alpha_{2}\right)$} & Low & $6.17 * 10^{6}$ & \multirow[b]{2}{*}{$V_{2}=8.19 * 10^{6}$} \\
\hline & Medium & $1.38 * 10^{6}$ & \\
\hline
\end{tabular}


Table 6: Results of baseline calibration with K6 and K1 Specifications

\begin{tabular}{|c|c|c|c|}
\hline A's effort & Observable Output & Compensation (\$) & $\begin{array}{l}\text { Maximum Value } \\
\text { Function (\$) }\end{array}$ \\
\hline \multirow{3}{*}{ Low $\left(\alpha_{1}\right)$} & Low & 235 & \multirow{3}{*}{$V_{1}=4.96 * 10^{6}$} \\
\hline & Medium & 235 & \\
\hline & High & 811 & \\
\hline \multirow[b]{2}{*}{$\operatorname{High}\left(\alpha_{2}\right)$} & Low & $5.2 * 10^{6}$ & \multirow[b]{2}{*}{$V_{2}=5.02 * 10^{6}$} \\
\hline & Medium & $1.39 * 10^{6}$ & \\
\hline
\end{tabular}

Table 7: Results of baseline calibration with K6 and K2 Specifications

\begin{tabular}{|l|l|l|l|}
\hline A's effort & Observable Output & Compensation (\$) & $\begin{array}{l}\text { Maximum Value } \\
\text { Function (\$) }\end{array}$ \\
\hline \multirow{3}{*}{ Low $\left(\alpha_{1}\right)$} & Low & 235 & \multirow{3}{*}{$V_{1}=7.94 * 10^{6}$} \\
\cline { 2 - 3 } & Medium & 235 & \\
\cline { 2 - 3 } & High & 811 & \multirow{3}{*}{$V_{2}=8.46 * 10^{6}$} \\
\hline \multirow{3}{*}{ High $\left(\alpha_{2}\right)$} & Low & $4.6^{*} 10^{6}$ & \\
\cline { 2 - 3 } & Medium & $1.39 * 10^{6}$ & \\
\cline { 2 - 3 } & High & $1.39 * 10^{6}$ & \\
\hline
\end{tabular}

Table 8: Results of baseline calibration with K6 and K4 Specifications

\begin{tabular}{|c|c|c|c|}
\hline A's effort & Observable Output & Compensation (\$) & $\begin{array}{l}\text { Maximum Value } \\
\text { Function (\$) }\end{array}$ \\
\hline \multirow{3}{*}{ Low $\left(\alpha_{1}\right)$} & Low & 235 & \multirow{3}{*}{$V_{1}=9.21 * 10^{6}$} \\
\hline & Medium & 235 & \\
\hline & High & 811 & \\
\hline \multirow[b]{2}{*}{$\operatorname{High}\left(\alpha_{2}\right)$} & Low & $5.2 * 10^{6}$ & \multirow[b]{2}{*}{$V_{2}=9.92 * 10^{6}$} \\
\hline & Medium & $1.39 * 10^{6}$ & \\
\hline
\end{tabular}


Table 9: Results of baseline calibration with K7 and K2 Specifications

\begin{tabular}{|c|c|c|c|}
\hline A's effort & Observable Output & Compensation (\$) & $\begin{array}{l}\text { Maximum Value } \\
\text { Function (\$) }\end{array}$ \\
\hline \multirow{3}{*}{ Low $\left(\alpha_{1}\right)$} & Low & 215 & \multirow{3}{*}{$V_{1}=7.94 * 10^{6}$} \\
\hline & Medium & 215 & \\
\hline & High & 203 & \\
\hline \multirow[b]{2}{*}{$\operatorname{High}\left(\alpha_{2}\right)$} & Low & $1.92 * 10^{7}$ & \multirow[b]{2}{*}{$V_{2}=8.6^{*} 10^{6}$} \\
\hline & Medium & $1.25^{*} 10^{6}$ & \\
\hline
\end{tabular}

Table 10: Results of baseline calibration with $K 7$ and $K 3$ Specifications

\begin{tabular}{|l|l|l|l|}
\hline A's effort & Observable Output & Compensation (\$) & $\begin{array}{l}\text { Maximum Value } \\
\text { Function (\$) }\end{array}$ \\
\hline \multirow{3}{*}{ Low $\left(\alpha_{1}\right)$} & Low & 215 & \multirow{2}{*}{$V_{1}=7.72 * 10^{6}$} \\
\cline { 2 - 3 } & Medium & 215 & \\
\cline { 2 - 3 } & High & 203 & \multirow{2}{*}{$V_{2}=8.32 * 10^{6}$} \\
\hline \multirow{3}{*}{ High $\left(\alpha_{2}\right)$} & Low & $1.92^{*} 10^{7}$ & \\
\cline { 2 - 3 } & Medium & $1.25^{*} 10^{6}$ & \\
\cline { 2 - 3 } & High & $1.25^{*} 10^{6}$ & \\
\hline
\end{tabular}

Table 11: Results of baseline calibration with $K 7$ and K4 Specifications

\begin{tabular}{|c|c|c|c|}
\hline A's effort & Observable Output & Compensation (\$) & $\begin{array}{l}\text { Maximum Value } \\
\text { Function (\$) }\end{array}$ \\
\hline \multirow{3}{*}{ Low $\left(\alpha_{1}\right)$} & Low & 215 & \multirow{3}{*}{$V_{1}=9.21 * 10^{6}$} \\
\hline & Medium & 215 & \\
\hline & High & 203 & \\
\hline \multirow{3}{*}{$\operatorname{High}\left(\alpha_{2}\right)$} & Low & $1.92 * 10^{7}$ & \multirow{3}{*}{$V_{2}=10.06^{*} 10^{6}$} \\
\hline & Medium & $1.25 * 10^{6}$ & \\
\hline & High & $1.25 * 10^{6}$ & \\
\hline
\end{tabular}




\section{References}

[1] Baker, George. (1992). "Incentive Contracts and Performance Measurement." Journal of Political Economy, 100(3), p. 598-614.

[2] Baker, George; Gibbons, Robert and Kevin J. Murphy. (1994). "Subjective Performance Measures in Optimal Incentive Contracts." Quarterly Journal of Economics, 109(2), p. 1125-1156.

[3] Baker, George; Gibbons, Robert and Kevin J. Murphy. (2002). "Relational Contracts and the Theory of the Firm." Quarterly Journal of Economics, 117(1), p. 39-84.

[4] Bakun, A. (1990). "Global Climate Change and Intensification of Coastal Ocean Upwelling." Science, 247, p. 198-201.

[5] Bakun, A. and S. J. Weeks. (2004). "Greenhouse Gas Buildup, Sardines, Submarine Eruptions and the Possibility of Abrupt Degradation of Intense Marine Upwelling Ecosystems." Ecology Letters, 7, p. 1015-1023.

[6] Barrett, Scott. (1994). "Self-Enforcing International Environmental Agreements." Oxford Economic Papers, 46, p. 878-894.

[7] Barrett, Scott. (2005). "Environment and Statecraft. The Strategy of Environmental Treaty Making." Oxford University Press.

[8] Beamish, R. J.; Benson, A. J.; Sweeting, R. M. and C. M. Neville. (2004). "Regimes and the history of the Major Fisheries off Canada's West Coast." Progress in Oceanography, 60, p. 355-385.

[9] Bjørndal, Trond; Kaitala, Veijo; Lindroos, Marko and Gordon R. Munro. (2000). "The Management of High Seas Fisheries." Annals of Operational Research, 94, p. 183-196.

[10] Block, B; Teo, S.; Walli, A.; Boustany, A.; Stokesbury, M; Farwell, C.; Weng, K.; Dewar, H and T. William. (2005). "Electronic Tagging and Population Structure of Atlantic Bluefin Tuna." Nature, 434, p. 1121-1127.

[11] Bolton, Patrick and Mathias Dewatripont. (2005). "Contract Theory." MIT Press.

[12] Brown, C. (2007). "Standardized Catch Rates of Bluefin Tuna Thunnus Thynnus, from the Rod and Reel/Handline Fishery off the Northeast US during 1980-2005." Col. Vol. Sci. Pap. ICCAT, 60, p. 1087-1108.

[13] Bull, Clive. (1987). "The Existence of Self-Enforcing Implicit Contracts." Quarterly Journal of Economics, 102(1), p. 147-159. 
[14] Carraro, Carlo and Domenico Siniscalco. (1993). "Strategies for the International Protection of the Environment." Journal of Public Economics, 52, p. 309-328.

[15] Cass-Calay, S. (2007). "Standardized Catch Rates of Large Bluefin Tuna (Thunnus thynnus) from the U.S. Pelagic Longline Fishery in the Gulf of Mexico and off the Florida East Coast During 1987-2005." Col. Vol. Sci. Pap. ICCAT, 60, p. 1070-1086.

[16] Chambers, Robert G. (2002). "Information, Incentives and the Design of Agricultural Policies" in the Handbook of Agricultural Economics, Vol. 2B: Agricultural and Food Policy, Eds. Gardner B. L. and G.C. Rausser.

[17] Chander, Parkash and Henry Tulkens. (1997). "The Core of an Economy with Multilateral Environmental Externalities." International Journal of Game Theory, 26, p. 379-401.

[18] Clark, C.W. and G.R. Munro. (1975). "The Economics of Fishing and Modern Capital Theory: A Simplified Approach." Journal of Environmental Economics and Management, 2, p. 96-106.

[19] Clarke, F.H. and G.R. Munro. (1987). "Coastal States, Distant Water Fishing Nations and Extended Jurisdictions; A Principal-Agent Analysis." Natural Resource Modeling, 2(1), p. 81-107.

[20] Clarke, F.H. and G.R. Munro. (1991). "Coastal States and Distant Water Fishing Nations: Conflicting views of the Future." Natural Resource Modeling, 5(3), p. 345-369.

[21] Cook, R. M. and M. R. Heath. (2005). "The Implications of Warming Climate for the Management of North Sea Demersal Fisheries." ICES Journal of Marine Science, 62, p. 1322 - 1326.

[22] FAO. (2007). " The State of World Fisheries and Aquaculture. 2006." Food and Agriculture Organization, United Nations.

[23] Fischer, Ronald D. and Leonard J. Mirman. (1996). "The Compleat Fish Wars: Biological and Dynamic Interactions." Journal of Environmental Economics and Management, 30, p. 34-42.

[24] Funaki, Yukihiko and Takehiko Yamato. (1999). "The Core of an Economy with a Common Pool Resource: A Partition Function Form Approach." International Journal of Game Theory, 28, p.157-171.

[25] Hannesson, Rognvaldur. (1995). "Sequential Fishing: Cooperative and Non-cooperative Equilibria." Natural Resource Modeling, 9(1), p. 51-59.

[26] Hannesson, Rognvaldur. (1997). "Fishing as a Supergame." Journal of Environmental Economics and Management, 32, p. 309-322. 
[27] ICCAT. (2004). "Supplemental Recommendation by ICCAT Concerning the Western Atlantic Bluefin Tuna Rebuilding Program." International Commission for the Conservation of Atlantic Tunas, Recommendation 0804 .

[28] ICCAT. (2008). "Report of the Standing Committee on Research and Statistics." International Commission for the Conservation of Atlantic Tunas, Madrid, Spain, September 29-October 3.

[29] ICCAT. (2010). "Report of the Standing Committee on Research and Statistics." International Commission for the Conservation of Atlantic Tunas, Madrid, Spain, October 4-8.

[30] ICCAT. (2010b). "Supplemental Recommendation by ICCAT Concerning the Western Atlantic Bluefin Tuna Rebuilding Program." International Commission for the Conservation of Atlantic Tunas, Recommendation 1003.

[31] Kaitala, Veijo and Marko Lindroos. (1998). "Sharing the Benefits of Cooperation in High Seas Fisheries: A Characteristic Function Game Approach." Natural Resource Modeling, 11, p. 275 - 299.

[32] Kaitala, Veijo and Gordon R. Munro. (1993). "The Management of High Sea Fisheries." Marine Resource Economics, 8, p. 313-329.

[33] Kaitala, Veijo and Gordon R. Munro. (1997). "The Conservation and Management of High Seas Fishery Resources Under the New Law of the Sea." Natural Resource Modeling, 10, p. 87-108.

[34] Kaitala Veijo and Marko Lindroos. (2004). "When to Ratify an Environmental Agreement: The Case of High Seas Fisheries." International Game Theory Review, 6(1), p. 55-68.

[35] Klein, Benjamin and Keith B. Leffler. (1981). "The Role of Market Forces in Assuring Contractual Performance." Journal of Political Economy, 89(41), p. $615-641$.

[36] Kwon, Oh Sang. (2006). "Partial International Coordination in the Great Fish War." Environmental and Resource Economics, 33(4), p. 463-483.

[37] Laffont, Jean-Jacques and David Martimort. (2002). "The Theory of Incentives. The Principal-Agent Model." Princeton University Press.

[38] Larkin, S.; Adams, C. and D. Lee. (2000). "Reported Trip Costs, Gross Revenues, and Net Returns for US Atlantic Pelagic Longline Vessels." Marine Fisheries Review, 62, p. 49-60.

[39] Laukkanen, Marita. (2003). "Cooperative and Non-cooperative Harvesting in a Stochastic Sequential Fishery." Journal of Environmental Economics and Management, 45, p. 454-473. 
[40] Laukkanen, Marita. (2005). "Cooperation in a Stochastic Transboundary Fishery: The Effects of Implementation Uncertainty versus Recruitment Uncertainty." Environmental 83 Resource Economics, 32, p. 389-405.

[41] Levhari, David and Leonard J. Mirman. (1980). "The Great Fish War: An example using a Dynamic Cournot-Nash Solution." Bell Journal of Economics, 11(1), p. 322-334.

[42] Li, Eric. (1998)."Cooperative High-Seas Straddling Stock Agreement as a Characteristic Function Game." Marine Resource Economics, 13, p. 247258.

[43] Lutcavage, M.; Brill, R.; Skomal, G.; Chase, B. and P. Howey. (1999). "Results of pop-up satellite tagging on spawning size class fish in the Gulf of Maine. Do North Atlantic bluefin tuna spawn in the Mid-Atlantic?" Can. J. Fish. Aquat. Sci., 56, p. 173-177.

[44] MacLeod, Bentley. (2003). "Optimal Contracting with Subjective Evaluation." American Economic Review, 93(1), p. 216-240.

[45] MacLeod, Bentley. (2007). "Reputations, Relationships and Contract Enforcement." Journal of Economic Literature, 45(3), p. 595-628.

[46] MacLeod, Bentley and J.M. Malcomson. (1989). "Implicit Contracts, Incentive Compatibility and Involuntary Unemployment." Econometrica, 57, p. $447-480$.

[47] Mather, F. J.; Mason J. M. and A. C. Jones. (1995). "Historical Document: Life History and Fisheries of Atlantic Bluefin Tuna." NOAA Technical Memorandum, NMFS-SEFSC-370, p. 165.

[48] McAllister, M. and T. Carruthers (2008). "2007 Stock Assessment and Projections for Western Atlantic Bluefin Tuna using a BSP and other SRA Methodology." Col. Vol. Sci. Pap. ICCAT, 62, p. 1206-1270.

[49] McAllister, M.; Neilson J. and C. Porch. (2008). "Discussion of the Alternative Explanations for the Relatively Low US Catches of Bluefin Tuna since 2004." Col. Vol. Sci. Pap. ICCAT, 62, p. 1298-1303.

[50] McKelvey, Robert. (1997). "Game-Theoretic Insights into the International Management of Fisheries." Natural Resource Modeling, 10(2), p. 129-171.

[51] McKelvey, Robert; Sandal, Leif and Stein Steinshamn. (2002). "Fish Wars on the High Seas: A Straddling Stock Competition Model." International Game Theory Review, 5(4), p. 327-345.

[52] McKelvey, Robert; Sandal, Leif and Stein Steinshamn. (2003). "Regional Fisheries Management on the High Seas: The Hit-and-Run Interloper Model." International Game Theory Review, 4(1), p. 53-69. 
[53] Miller, K. A. and G. B. Munro. (2004). "Climate and Coopertation: A new Perspective on the Management of Shared Fish Stock." Marine Resource Economics, 19, p. 367-393.

[54] Miller, K. A.; Munro, G. B.; McKelvey, R. and Peter Tyedmers. (2000). "Climate, Uncertainty and the Pacific Salmon Treaty: Insights on the Harvest Management Game." IIFET 2000 Proceedings.

[55] Munro, Gordon R. (1979). "The Optimal Management of Transboundary Renewable Resources." Canadian Journal of Economics, 12(3), p. 355-376.

[56] Munro, Gordon R. (1987). "The Management of Shared Fishery Resources under Extended Jurisdiction." Marine Resource Economics, 3(4), p. 271296.

[57] Munro, Gordon R. (1990). "The Optimal Management of Transboundary Fisheries: Game Theoretic Considerations." Natural Resource Modeling, 4(4), p. 403-426.

[58] Munro, Gordon R. (2001). "The United Nations Fish Stocks Agreement of 1995: History and problems of implementation." Marine Resource Economics, 15 , p. 265-280.

[59] Munro, Gordon R. (2007). "Internationally Shared Fish Stocks, the High Seas, and Property Rights in Fisheries." Marine Resource Economics, $22(4)$, p. $425-443$.

[60] Munro, Gordon R. (2011). "Regional Fisheries Management Organizations and the New Member Problem: From Theory to Policy." Paper presented in the 8th Meeting on Game Theory and Practice Dedicated to Global and International Issues, University of California, Riverside, California, July $11-12$.

[61] Munro, Gordon R.; Van Houtte, A. and R. Willmann. (2004). "The Conservation and Management of Shared fish stocks: Legal and Economic Aspects." FAO Fisheries Technical Paper No. 465, Rome, Italy.

[62] Nash, John. (1950). "The Bargaining Problem." Economtrica, 18, p. 155162.

[63] Nash, John. (1951). "Non-Cooperative Games." Annals of Mathematics, $54(2)$, p. $286-295$.

[64] Nash, John. (1953). "Two-person Cooperative Games." Econometrica, 21, p. $128-140$.

[65] Neilson, J. D.; Paul, S. D. and M. Ortiz. (2007). "Indices of Stock Status Obtained from the Canadian Bluefin Tuna Fishery." Col. Vol. Sci. Pap. ICCAT, 60, p. 976-1000. 
[66] NOAA. (2008). "Stock Assessment and Fishery Evaluation (SAFE) Report for Atlantic Highly Migratory Species." National Marine Fisheries Service, National Oceanic and Atmospheric Administration.

[67] NOAA. (2010). "Status Review Report of Atlantic Bluefin Tuna (Thunnus thynnus)." Prepared by the Atlantic Bluefin Tuna Status Review Team for the National Marine Fisheries Service, National Oceanic and Atmospheric Administration.

[68] NOAA. (2011). "International Agreements Concerning Living Marine Resources of Interest to NOAA Fisheries." Office of International Affairs, National Marine Fisheries Service, National Oceanic and Atmospheric Administration.

[69] NOAA. (2011b). "Stock Assessment and Fishery Evaluation (SAFE) Report for Atlantic Highly Migratory Species." National Oceanic and Atmospheric Administration, National Marine Fisheries Service.

[70] Perry, A. L.; Low, P. J.; Ellis, J. R and J. D. Reynolds. (2005). "Climate Change and Distribution Shifts in Marine Fishes." Science, 308, p. 19121915 .

[71] Pintassilgo, Pedro. (2003). "A Coalition Approach to the Management of High Seas Fisheries in the Presence of Externalities." Natural Resource Modeling, 16, p. 175-197.

[72] Pintassilgo, Pedro and Marko Lindroos. (2008). "Coalition Formation in High Seas Fisheries: A Partition Function Approach." International Game Theory Review ,10, p. 303-317.

[73] Rogerson, W.P. (1985). "Repeated Moral Hazard." Econometrica, 53, p. 69-76.

[74] Secor, D. (2007). "Do some Atlantic Bluefin Tuna Skip Spawning?" Col. Vol. Sci. Pap. ICCAT, 60, p. 1141-1153.

[75] Telser, L.G. (1980). "A Theory of Self-Enforcing Agreements." Journal of Business, 53(1), p. 27-44.

[76] Turner, S.C. and V.R. Restrepo. (1994). "A Review of the Growth Rate of West Alantic Bluefin tuna Thunnus thynnus Estimated from Marked and Recaptured fish." SCRS/93/65, Collective Volume of Scientific Papers, ICCAT, 42, p. 170-172.

[77] United Nations. (1928). "United Nations Convention on the Law of the Sea." December 10.

[78] United Nations. (1995). "Agreement for the Implementation of the Provisions of the United Nations Convention on the Law of the Sea of 10 
December 1982 Relating to the Conservation and Management of Straddling Fish Stocks and Highly Migratory Fish Stocks." UN Doc. A/Conf. $164 / 37$.

[79] United Nations. (2006). "Review Conference on the Agreement for the Implementation of the Provisions of the United Nations Convention on the Law of the Sea of 10 December 1982 Relating to the Conservation and Management of Straddling Fish Stocks and Highly Migratory Fish Stocks." UN Doc. A/Conf. 210/15.

[80] US Federal Register. (2009). Federal Register Vol. 74, No. 103, June 1.

[81] US Federal Register. (2010). Federal Register Vol. 75, No. 105, June 2.

[82] Vilchis, L. I.; Tegner, M. J.; Moore, J. D.; Friedman, C. S.; Riser, K. L.; Robbins, T. T. and P. K. Dayton. (2005). "Ocean Warming Effects on Growth, Reproduction and Survivorship of Southern California Abalone/" Ecological Applications, 15(2), p. 469-480.

[83] Wilson, S.G.; Lutcavage, M.E.; Brill, R.W.; Genovese, M.P.; Cooper, A. B. and A. Everly. (2005). "Movements of Bluefin Tuna (Thunnus thynnus) in the Northwestern Atlantic Ocean Recorded by Pop-up Satellite Archival Tags." Marine Biology, 146, p. 409- 423.

[84] Yi, Sang-Seung. (1997). "Stable Coalition Structures with Externalities." Games and Economic Behavior, 20, p. 201-237. 